\title{
Georgia: Sixth Review Under the Stand-By Arrangement and Requests for Modification of Performance Criteria, Waiver of Nonobservance of Performance Criterion, Waiver of Applicability of Performance Criterion, and Rephasing of Purchase-Staff Report; Staff Statement; Press Release on the Executive Board Discussion; and Statement by the Executive Director for Georgia.
}

In the context of the sixth review under the stand-by arrangement and requests for modification of performance criteria, waiver of nonobservance of performance criterion, waiver of applicability of performance criterion, and rephasing of purchase, the following documents have been released and are included in this package:

- $\quad$ The staff report for the Sixth Review Under the Stand-By Arrangement and Requests for Modification of Performance Criteria, Waiver of Nonobservance of Performance Criterion, Waiver of Applicability of Performance Criterion, and Rephasing of Purchase, prepared by a staff team of the IMF, following discussions that ended on May 13, 2010, with the officials of Georgia on economic developments and policies. Based on information available at the time of these discussions, the staff report was completed on June 24, 2010. The views expressed in the staff report are those of the staff team and do not necessarily reflect the views of the Executive Board of the IMF.

- A staff statement of July 9, 2010 updating information on recent developments.

- $\quad$ A Press Release summarizing the views of the Executive Board as expressed during its July 9, 2010 discussion of the staff report that completed the request and/or review.

- $\quad$ A statement by the Executive Director for Georgia.

The documents listed below have been or will be separately released.

Letter of Intent sent to the IMF by the authorities of Georgia*

Technical Memorandum of Understanding*

*Also included in Staff Report

The policy of publication of staff reports and other documents allows for the deletion of market-sensitive information.

\author{
Copies of this report are available to the public from \\ International Monetary Fund • Publication Services \\ $70019^{\text {th }}$ Street, N.W. • Washington, D.C. 20431 \\ Telephone: (202) 623-7430 • Telefax: (202) 623-7201 \\ E-mail: publications@imf.org Internet: http://www.imf.org
}

\section{International Monetary Fund Washington, D.C.}




\title{
INTERNATIONAL MONETARY FUND
}

\section{GEORGIA}

\section{Sixth Review Under the Stand-By Arrangement and Requests for Modification of Performance Criteria, Waiver of Nonobservance of Performance Criterion, Waiver of Applicability of Performance Criterion, and Rephasing of Purchase}

\author{
Prepared by the Middle East and Central Asia Department \\ (In consultation with other departments)
}

Approved by David Owen (MCD) and Dominique Desruelle (SPR)

June 24, 2010

- Background: The Fifth Review under the Stand-By Arrangement (SBA) was concluded on March 19, 2010. Access under the SBA is SDR 747.1 million (497.1 percent of quota), of which SDR 527.1 million has been disbursed. The arrangement expires June $14,2011$.

- Program Strategy: While the economic recovery is gaining ground, renewed foreign exchange market pressures have required faster exchange rate adjustment and a tightening of fiscal and monetary policies, so as to ensure timely external adjustment.

- Team: E. Gardner (head and Senior Resident Representative), D. Simard, A. Luca (all MCD), L. Eyraud (FAD), I. Halikias (SPR), and N. Sharashidze (Resident Representative Office).

- Exchange Rate Regime: The regime is classified as "other managed." A multiple currency practice arises from the fact that the official exchange rate used by the government may differ by more than 2 percent from freely determined market rates.

- Statistics: Economic data are broadly adequate for surveillance and program monitoring. Georgia participates in the GDDS and subscribed to the SDDS in May 2010. 
Contents $\quad$ Page

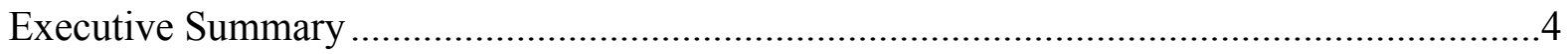

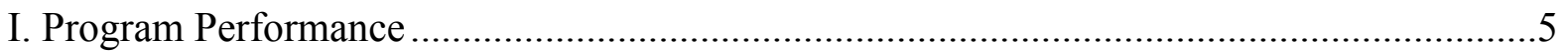

II. Recent Developments and Outlook for 2010 ........................................................6

III. Policies for 2010, the Medium-Term Outlook, and the Exit Strategy ............................ 10

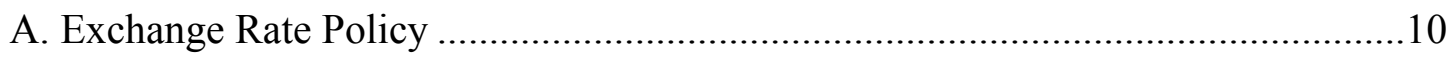

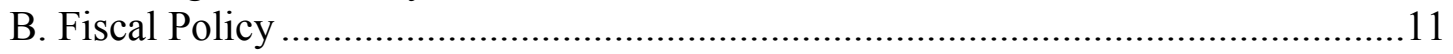

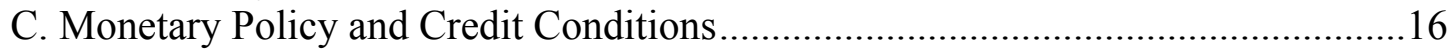

D. Medium-Term Outlook and the Exit Strategy ..................................................19

E. Risks to the Medium-Term Strategy .............................................................20

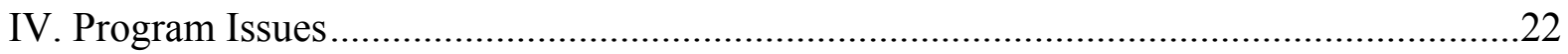

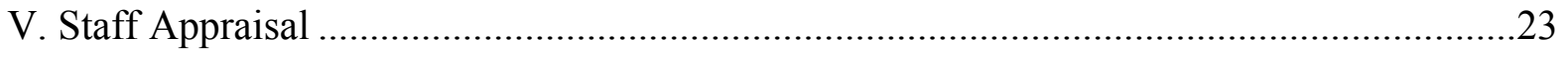

\section{Tables}

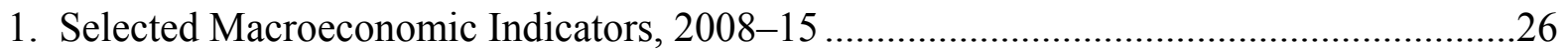

2. Annual General Government Operations, 2009-15 .....................................................27

3. Quarterly General Government Operations, 2009-10 …...........................................28

4. Summary Balance of Payments, 2008-15 ....................................................................29

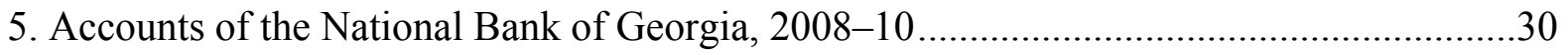

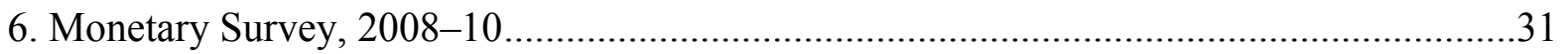

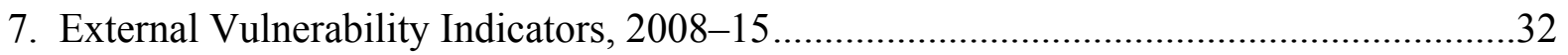

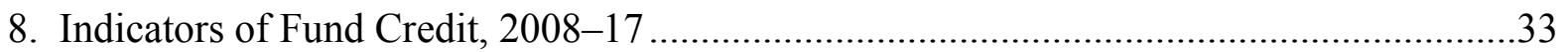

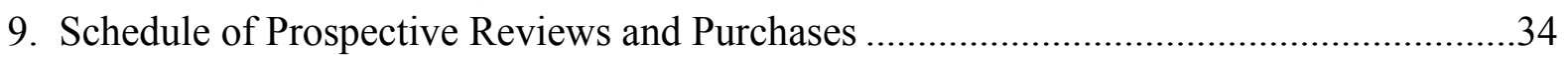

10. External Financing Requirements and Sources, 2008-15 ...........................................35

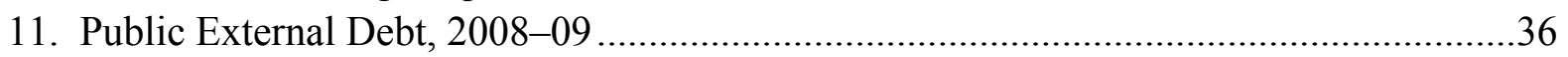

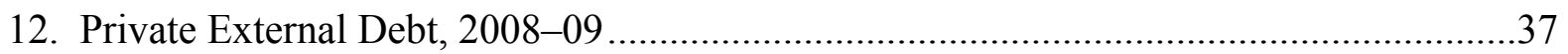

13a. External Debt Sustainability Framework, Baseline Scenario, 2007-30 ........................38

13b. Sensitivity Analysis for Key Indicators of Public and Publicly-Guaranteed External

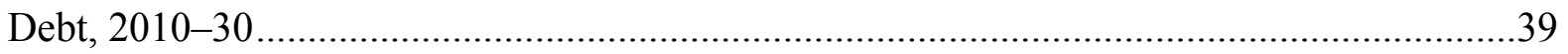

14a. Public Sector Debt Sustainability Framework, Baseline Scenario, 2007-30 ................41

14b. Sensitivity Analysis for Key Indicators of Public Debt, 2010-30 ..............................42

\section{FIGURES}

1a. Indicators of Public and Publicly-Guaranteed External Debt under Alternative

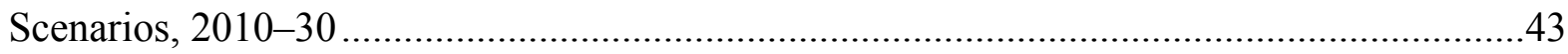

1b. Indicators of Public Debt under Alternative Scenarios, 2010 -30 .................................44 


\section{BOXES}

1. Measures to Enhance Monetary Policy Effectiveness and Encourage Financial

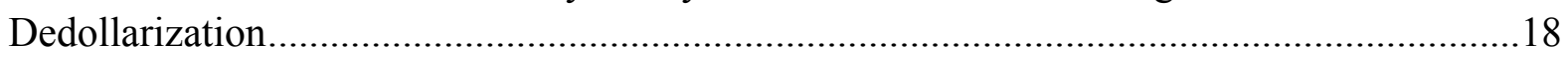

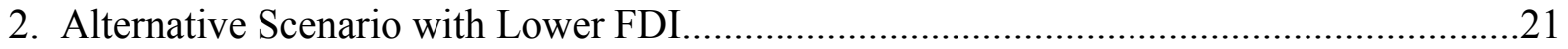

AtTachments

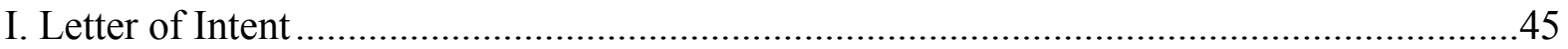

II. Revised Technical Memorandum of Understanding ......................................................56 


\section{EXECUTIVE SUMMARY}

Real GDP growth for 2010 has been revised up from 2 to $4 \frac{1}{2}$ percent, based on two consecutive quarters of solid expansion of economic activity and the resumption of credit growth supported by a gradual lowering of interest rates. This projection is tempered by higher external risks associated with problems in the Euro zone. End-March quantitative performance criteria (NIR, NDA, and the fiscal deficit) were met with ample margins, and the fiscal deficit and NDA targets of end-June are expected to be met. However, the end-June NIR will likely be missed as a result of a delayed exchange rate adjustment to persistent pressures in April-May. Since then, fiscal and monetary policies have been tightened and the exchange rate has been allowed to depreciate faster, thus buttressing the exit strategy and ensuring that the economic recovery is externally sustainable.

The cyclical rebound is being used to achieve a faster pace of deficit reduction (from 9.2 percent of GDP in 2009 to 6.3 percent in 2010). Notwithstanding some increase in spending, the fiscal consolidation strategy has been reinforced by the planned introduction of new revenue measures in 2010-11, an explicit expenditure cap for the rest of 2010, and the decision to postpone implementation of a referendum requirement on tax increases. A tightening of monetary policy in June was warranted, and will likely need to be followed by additional interest rate increases to preserve price and external stability.

Successful program execution should improve Georgia's prospects for re-accessing private capital markets. The exit strategy's reliance on a substantial pick up of private capital inflows, and FDI in particular, remains a risk factor. 


\section{Program Performance}

Georgia: Quantitative Performance Criteria (PC) and Indicative Targets, March-June 2010

\begin{tabular}{|c|c|c|c|c|c|}
\hline & \multicolumn{5}{|c|}{ Cumulative Change from End-December 2009} \\
\hline & \multicolumn{3}{|c|}{ Mar-10 } & \multicolumn{2}{|c|}{ Jun-10 } \\
\hline & $\mathrm{PC}$ & Adjusted PC & Actual & $\mathrm{PC}$ & Proj. \\
\hline & \multicolumn{5}{|c|}{ (In millions of lari) } \\
\hline \multirow[t]{2}{*}{ Ceiling on cash deficit of the general government } & 333 & & 96 & 737 & 467 \\
\hline & \multicolumn{5}{|c|}{ (In millions of lari) } \\
\hline \multirow[t]{2}{*}{ Ceiling on net domestic assets (NDA) of the NBG 1/ } & 418 & 397 & 135 & 606 & 553 \\
\hline & \multicolumn{5}{|c|}{ (In millions of U.S. dollars) } \\
\hline Floor on net international reserves (NIR) of the NBG 1/ & 781 & 793 & 897 & 685 & 639 \\
\hline \multirow[t]{3}{*}{ Ceiling on accumulation of external arrears $2 /$} & 0 & & 0 & 0 & 0 \\
\hline & Ind. Target & & Actual & Ind. Target & Proj. \\
\hline & \multicolumn{5}{|c|}{ (In millions of U.S. dollars) } \\
\hline $\begin{array}{l}\text { Ceiling on contracting or guaranteeing of new nonconcessional } \\
\text { external debt by the public sector }\end{array}$ & 850 & & 226 & 850 & 347 \\
\hline
\end{tabular}

Sources: Georgian authorities; and Fund staff estimates.

1/Actual figures and quantitative targets are based on program exchange rates.

$2 /$ The continuous performance criterion for external arrears is defined in paragraph 17 of the TMU.

1. All end-March program targets were met:

- NIR was well above target, as foreign exchange intervention was kept below agreed limits, despite a shortfall in privatization revenues and project loans.

- NDA was comfortably below the ceiling due to lower net lending to banks and higher accumulation of government deposits.

- The fiscal deficit was below the ceiling by a wide margin (1.2 percent of annual GDP) owing to a revenue windfall from higher growth and underexecution of spending - explained in part by implementation delays of foreign-financed capital projects.

2. End-June program targets are expected to be met, with the exception of the NIR target, which will likely be missed due to delayed exchange rate adjustment to pressures in April-May. Corrective measures have been taken to address the underlying imbalance. 


\section{RECENT DEVELOPMENTS AND OUTLOOK FOR 2010}

3. The orderly conclusion of the May 30 municipal elections removed uncertainty that was weighing on confidence. The ruling party retained control of Tbilisi and major cities.

\section{Real GDP growth for $\mathbf{2 0 1 0}$ has been} revised up from 2 to $4 \frac{1}{2}$ percent, on the back of two consecutive quarters of economic

expansion. While there are few direct spillover risks from instability in the Euro zone, external risks have increased. CPI inflation dropped to 4 percent in May, but is expected to stabilize at around 5 percent.

\section{Despite the acceleration of economic} activity, the current account deficit in the first quarter was lower than expected due to improved terms of trade, while private capital inflows remained low. The 2010 current account deficit is now projected at 12.6 percent of GDP. FDI inflows in the first quarter were very weak (\$76 million), prompting a downward revision of annual FDI inflows to below the level of 2009. Banks are expected to generate net financial inflows as they repatriate foreign assets to meet recently announced reserve requirements on external liabilities.
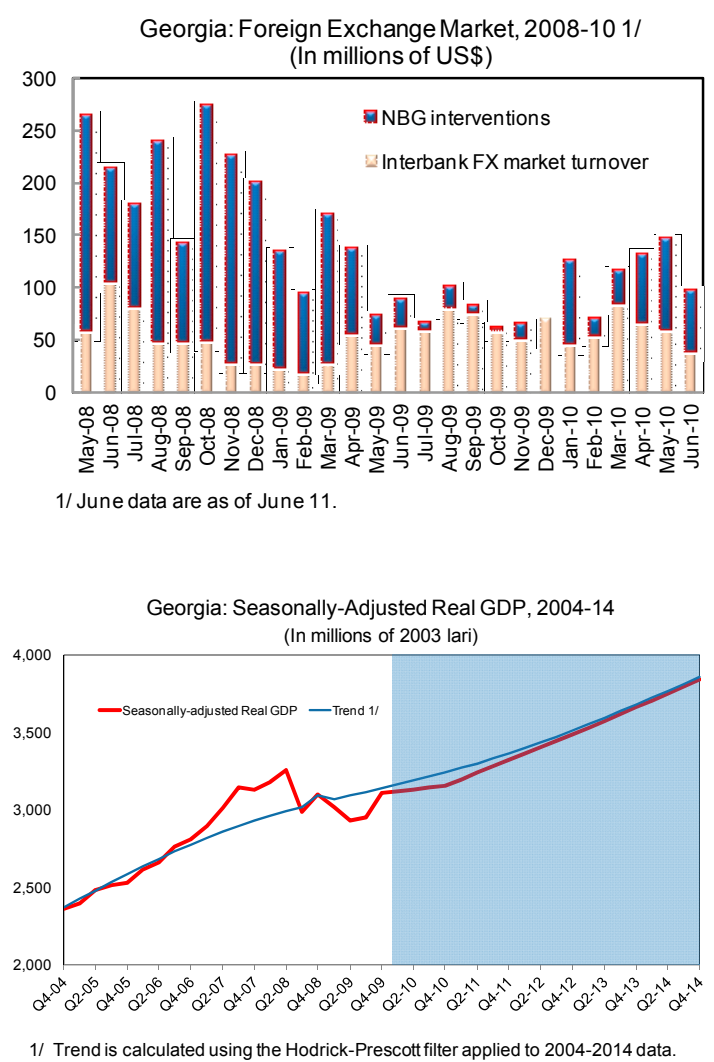

Georgia: Indicators of Economic Activity, March 2008-May 2010 (3-month moving average, $y$-o-y change in percent)
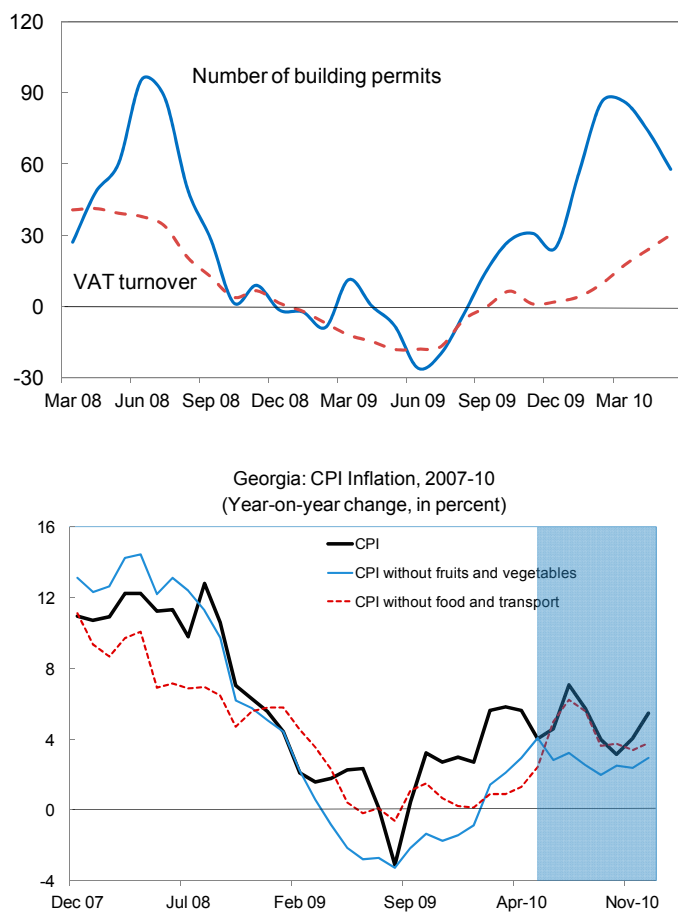

Sources: Georgian authorities; and Fund staff estimates and projections. 


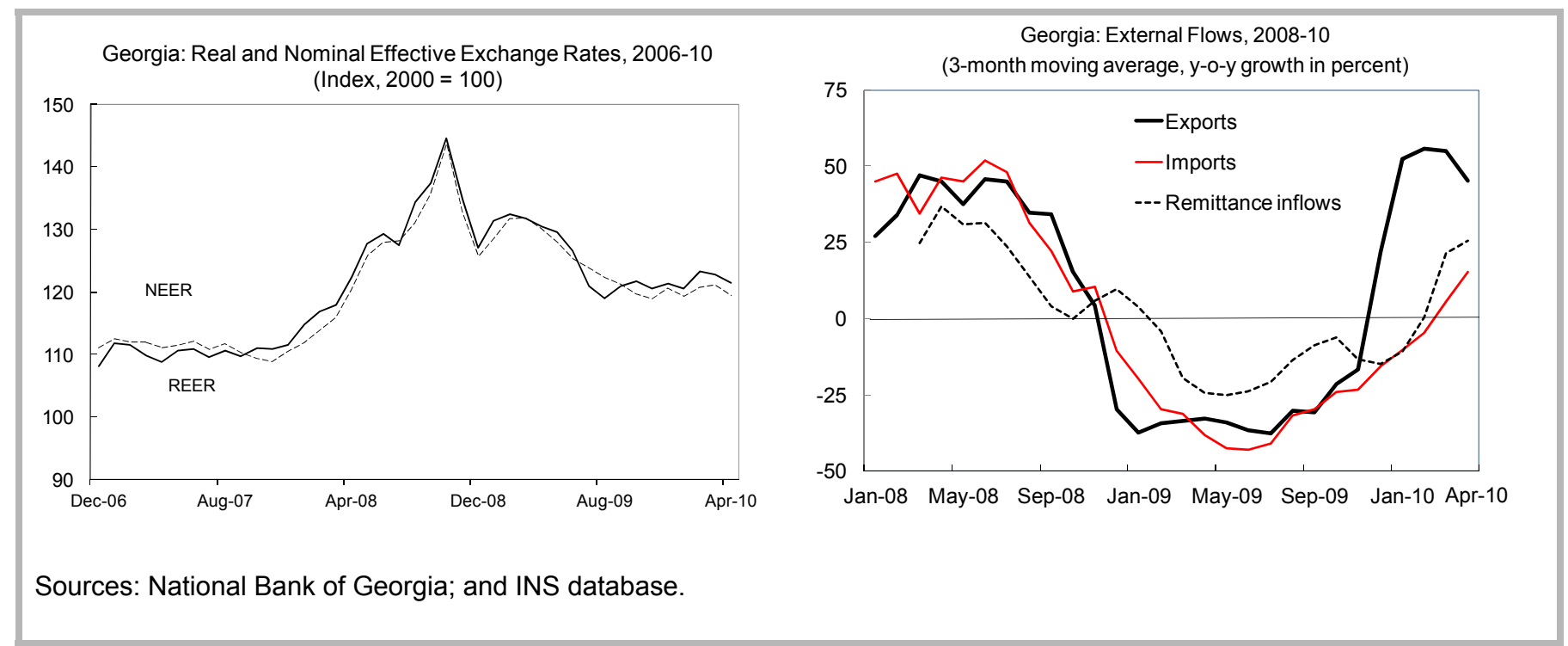

6. There are signs of a nascent recovery of credit growth, supported by a gradual lowering of deposit and lending rates over the past year. All of the growth in lending has been in local currency. The banks' balance sheets are cushioned by ample levels of capital and provisioning, but the high level of NPLs remains a risk factor.

Georgia: Selected Monetary and Financial Soundness Indicators, 2008-10

\begin{tabular}{|c|c|c|c|c|c|c|c|c|c|c|c|}
\hline & \multicolumn{4}{|c|}{2008} & \multicolumn{4}{|c|}{2009} & \multicolumn{3}{|c|}{2010} \\
\hline & Mar. & Jun. & Sep. & $\overline{\text { Dec. }}$ & Mar. & Jun. & Sep. & Dec. & Mar. & Apr. & May \\
\hline Deposit dollarization (in percent) & 60.9 & 60.0 & 61.7 & 75.7 & 75.2 & 73.2 & 72.6 & 73.2 & 71.0 & 70.1 & 71.3 \\
\hline Loan-to-deposit ratio (in percent) & 129.4 & 135.7 & 147.0 & 155.9 & 165.3 & 165.4 & 143.0 & 124.2 & 124.7 & 124.2 & 125.7 \\
\hline Credit-to-GDP ratio (in percent) & 28.5 & 30.0 & 29.2 & 31.9 & 30.7 & 30.2 & 29.6 & 29.3 & 29.7 & 30.5 & 30.1 \\
\hline Capital adequacy ratio (in percent) $1 /$ & 17.5 & 15.7 & 17.0 & 13.9 & 15.2 & 17.6 & 20.2 & 19.1 & 18.3 & 18.0 & 17.6 \\
\hline Capital adequacy ratio (in percent) $2 /$ & & & & 24.0 & 23.6 & 27.1 & 27.4 & 25.6 & 24.6 & 24.1 & 24.1 \\
\hline Liquidity ratio (in percent) $3 /$ & 36.8 & 33.3 & 30.4 & 28.3 & 30.8 & 31.4 & 40.6 & 39.1 & 41.6 & 39.8 & 38.6 \\
\hline Nonperforming loans (in percent of total loans) 4 / & 3.0 & 3.4 & 9.9 & 12.8 & 15.2 & 18.8 & 18.2 & 17.9 & 17.1 & 17.3 & 17.6 \\
\hline Nonperforming loans (in percent of total loans) 5 / & & & & 4.1 & 5.4 & 7.1 & 8.3 & 6.3 & 6.6 & 6.5 & 7.4 \\
\hline Loans collateralized by real estate (in percent of total loans) & 43.6 & 40.5 & 41.6 & 43.6 & 46.4 & 48.0 & 49.1 & 55.5 & 55.5 & 54.4 & 54.5 \\
\hline Loans in foreign exchange (in percent of total loans) & 65.9 & 64.9 & 67.5 & 72.8 & 75.3 & 77.3 & 77.6 & 76.9 & 75.9 & 75.1 & 74.3 \\
\hline Specific provisions (in percent of total loans) & 1.9 & 2.2 & 4.7 & 6.0 & 7.5 & 9.4 & 10.2 & 9.7 & 9.4 & 9.0 & 9.5 \\
\hline Net foreign assets (in percent of total assets) & -17.2 & -20.2 & -21.6 & -19.6 & -22.5 & -24.4 & -18.4 & -14.9 & -15.1 & -14.4 & -15.1 \\
\hline Net open foreign exchange position (in percent of regulatory capital) & 3.5 & 1.5 & 1.5 & 1.7 & 9.1 & 8.8 & 10.4 & 1.8 & 4.3 & 4.2 & 1.5 \\
\hline Return on equity (cumulative through the year, annualized) $6 /$ & 10.2 & 8.9 & -2.9 & -12.6 & -7.6 & -8.4 & -7.3 & -4.3 & 4.0 & 4.8 & 4.5 \\
\hline
\end{tabular}

Sources: National Bank of Georgia; and Fund staff estimates.

1/ National definition. Risk weight to forex loans was reduced from 200 to 175 percent in September 2008, and to 150 percent in August 2009. 2/ Basel I definition

3/ Ratio of liquid assets to 6-month and shorter maturity liabilities.

4/ National definition: NPLs are defined as loans in substandard, doubtful, and loss loan categories.

5/ IMF definition.

6/ Pre tax. 


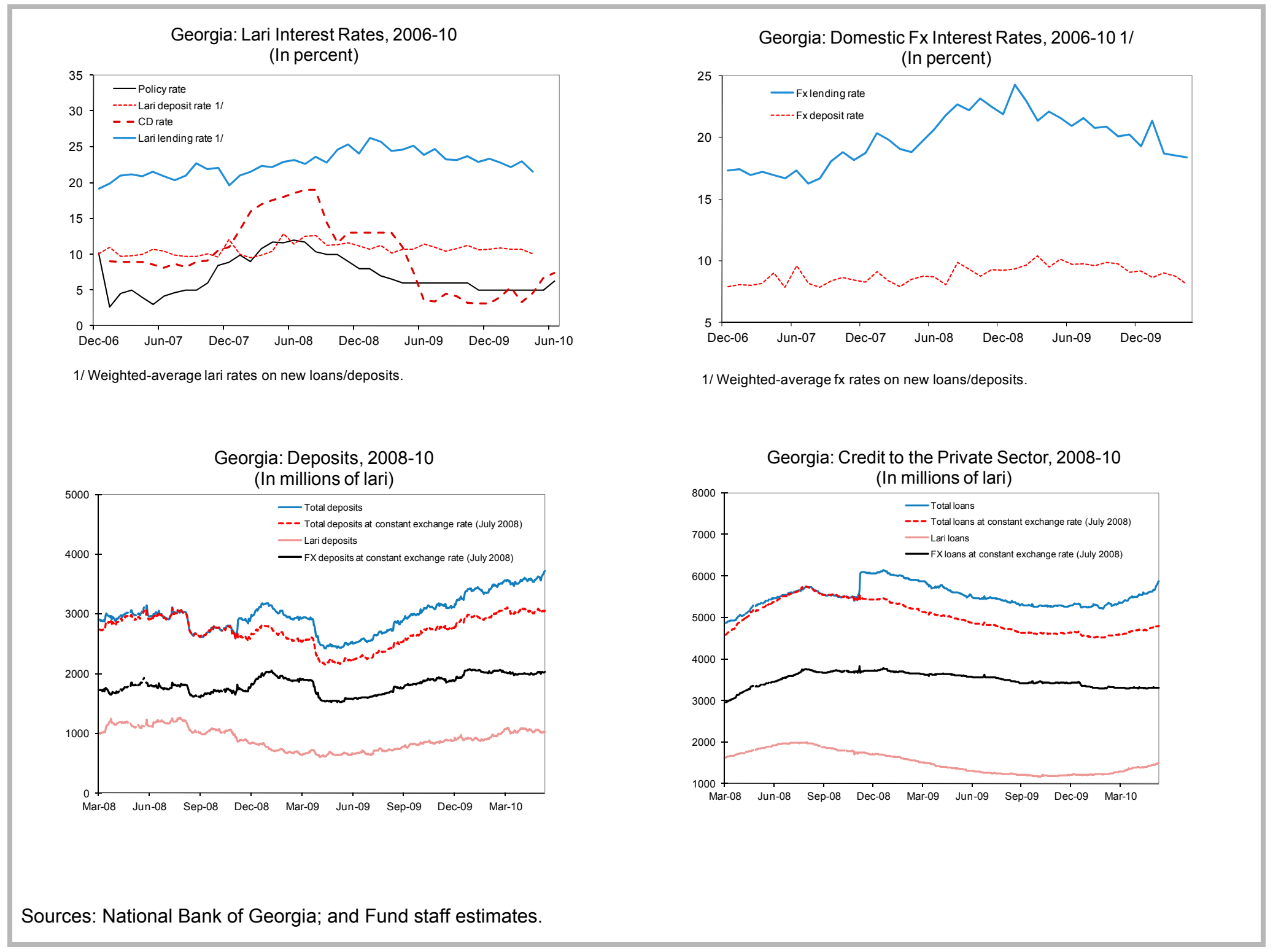

$\infty$ 


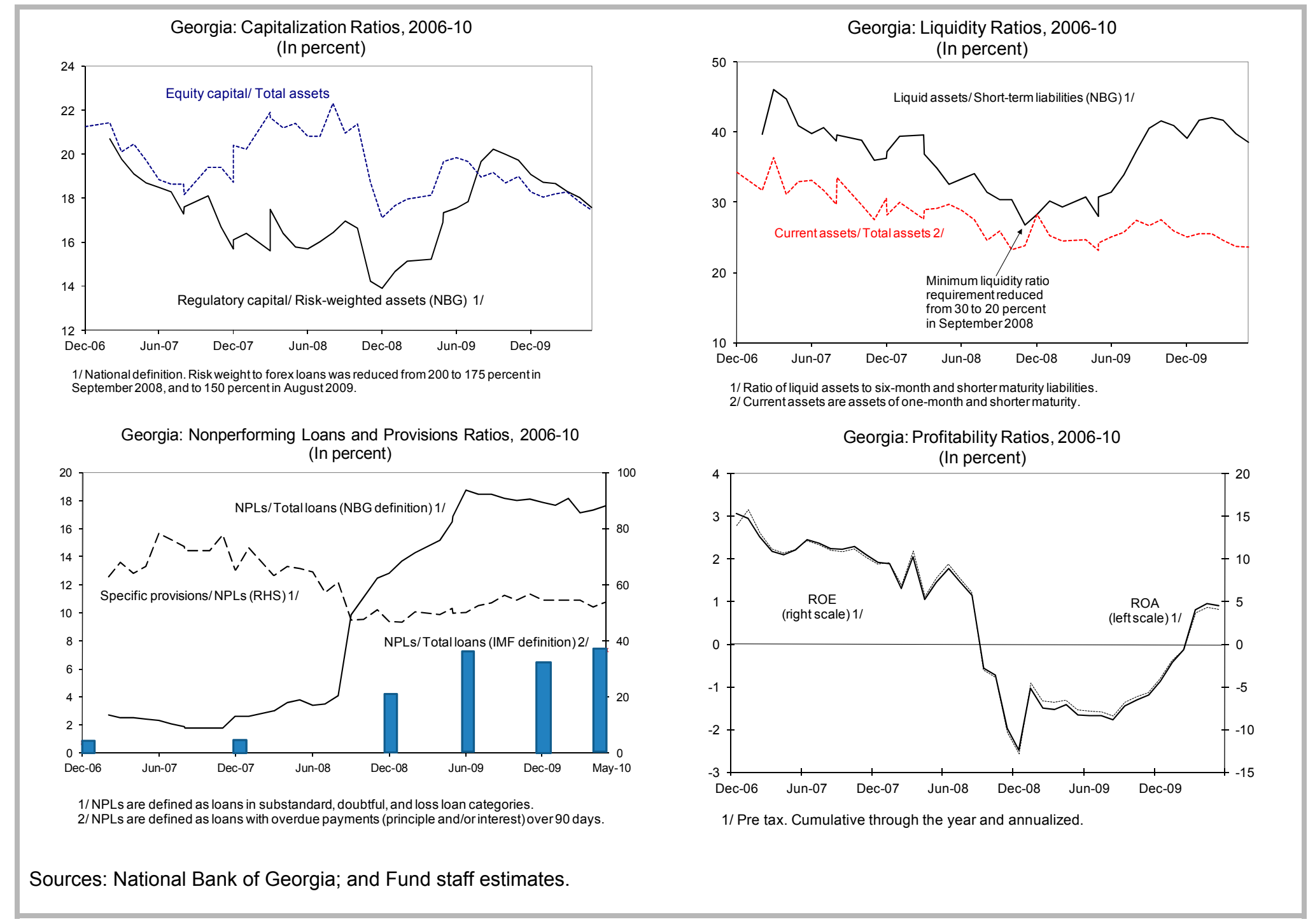

6 


\section{Policies for 2010, the Medium-Term Outlook, AND the EXIt StrategY `}

Based on an expected easing of seasonal foreign exchange market pressures, policy discussion in early May focused on consolidating the exit strategy in the context of an improved growth outlook.

However, the persistence of exchange rate pressures and the related downward revision of projected FDI inflows in 2010, required policies to be revisited in June in the direction of faster fiscal and exchange rate adjustments, and monetary tightening.

\section{A. Exchange Rate Policy}

7. Through April 2010, the exchange rate was managed flexibly, as evidenced by the lari's depreciation by 5 percent since the beginning of the year vis-à-vis the U.S. dollar (the currency of reference). In the face of seasonal foreign exchange market pressures, exchange rate policy was rebalanced in favor of more depreciation and less intervention, compared with the same period in 2009.

8. However, as exchange rate pressures persisted into May, the authorities increased intervention to contain the rate of
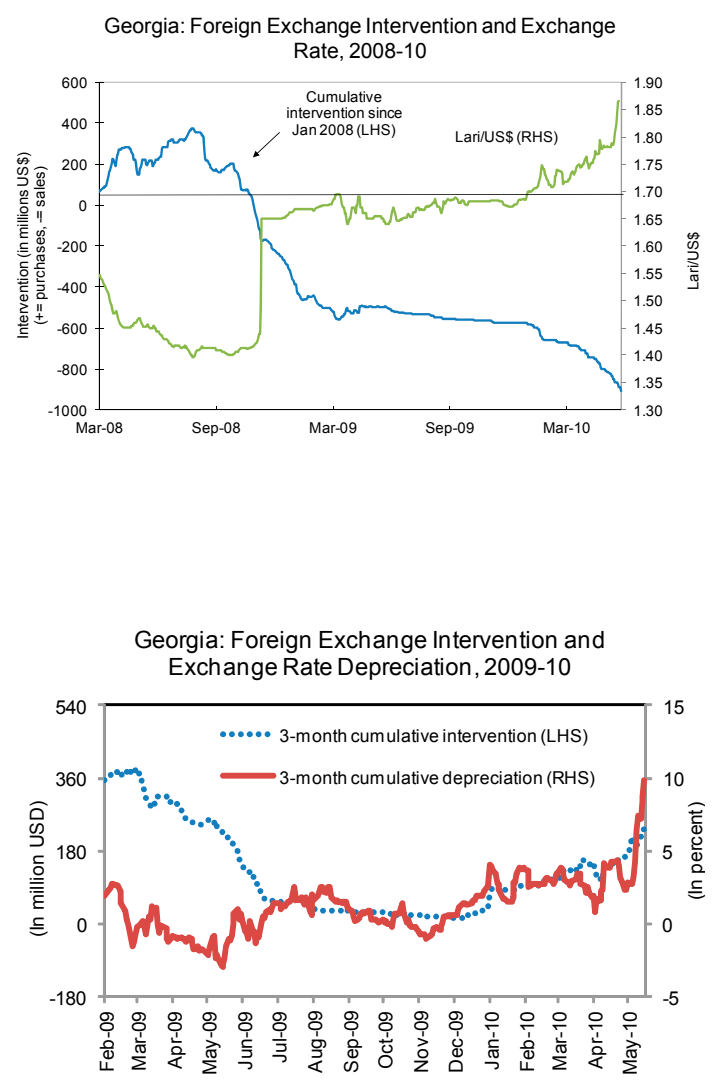

Sources: Georgian Authorities; and Fund staff estimates. depreciation, and will, as a result, likely fail to observe end-June NIR target. This policy choice reflected their view that pre-election uncertainty was driving pressures, which would reverse in June. Eventually, with signs of weaker-than-expected FDI inflows, the authorities acknowledged that the imbalance in the foreign exchange market had a structural component, which would require faster exchange rate adjustment. More expansionary fiscal and monetary policies in the second quarter of the year were recognized as additional contributing factors. 
9. On June 4, the authorities took action by reducing considerably the volume of dollars offered at auction. The resulting rapid movement in the rate- which depreciated by 8 percent by the end of the day-required some follow up intervention to stabilize public expectations. As of June 23, cumulative depreciation vis-à-vis the U.S. dollar was 10 percent since the beginning of the year, and market conditions had stabilized.

\section{To reinforce exchange rate flexibility} going forward, the authorities plan to limit intervention and the frequency of the auctions to once a week (LOI, $\uparrow 25)$. These two factors combined should promote foreign exchange trading within the market and thus ensure faster and smoother exchange rate adjustment. Foreign exchange inflows are expected to pick up due to seasonal factors (tourism and remittances) and an acceleration of private capital inflows in the second half of the year, riding on stronger GDP growth and the orderly conclusion of local elections, as well as a stronger privatization drive.

11. Under the revised program, the projected shortfall in private capital inflows relative to the fifth review (by US\$276 million, or 2.6 percent of GDP) is more than offset by improved current flows and higher official transfers. Despite the higher GDP growth, the current account deficit is revised downward by US\$189 million, mainly on account of improved terms of trade.

\section{B. Fiscal Policy}

12. Based on preliminary information, the budget deficit target of end-June will be met. Revenues are projected to come out above target on account of stronger GDP growth. Spending should come out below the program projection, even with some catch up relative to the underexecution of the first quarter. The increase in spending in the second quarter (which could exceed 20 percent over the first quarter) contributed to the foreign exchange market

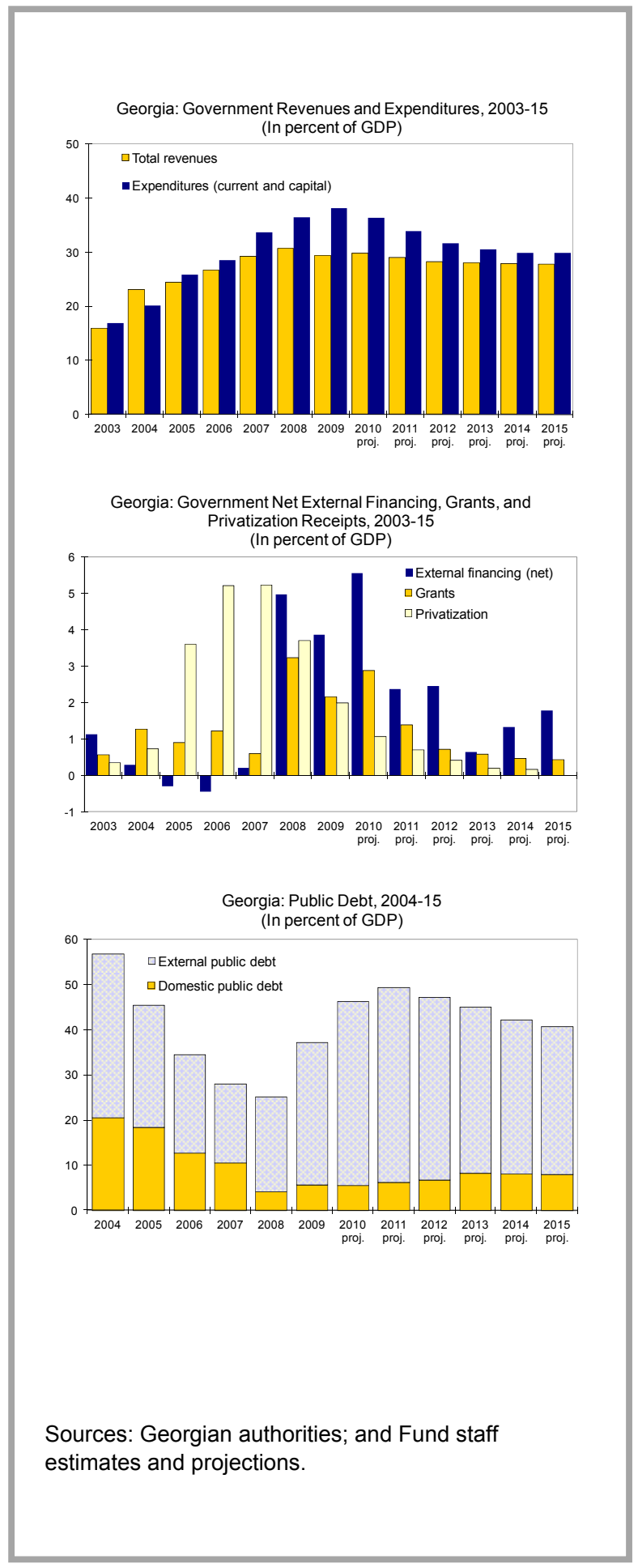


pressures observed in May.

13. Given the efforts already undertaken to reduce the deficit in $\mathbf{2 0 1 0}$, the authorities initially considered that the revenue gain expected in 2010 on account of higher growth should be largely used to finance some of the more pressing capital projects. The authorities underscored the strict wage bill containment and cuts in administrative costs underlying the 2010 adjustment. In the face of large unmet needs, the draft supplementary budget prepared in April allocated 85 percent of the projected revenue gain to new spending authorizations $(+1.1$ percent of GDP). This increase in spending allocations covered replenishment of reserve funds which were depleted by emergency spending due to natural

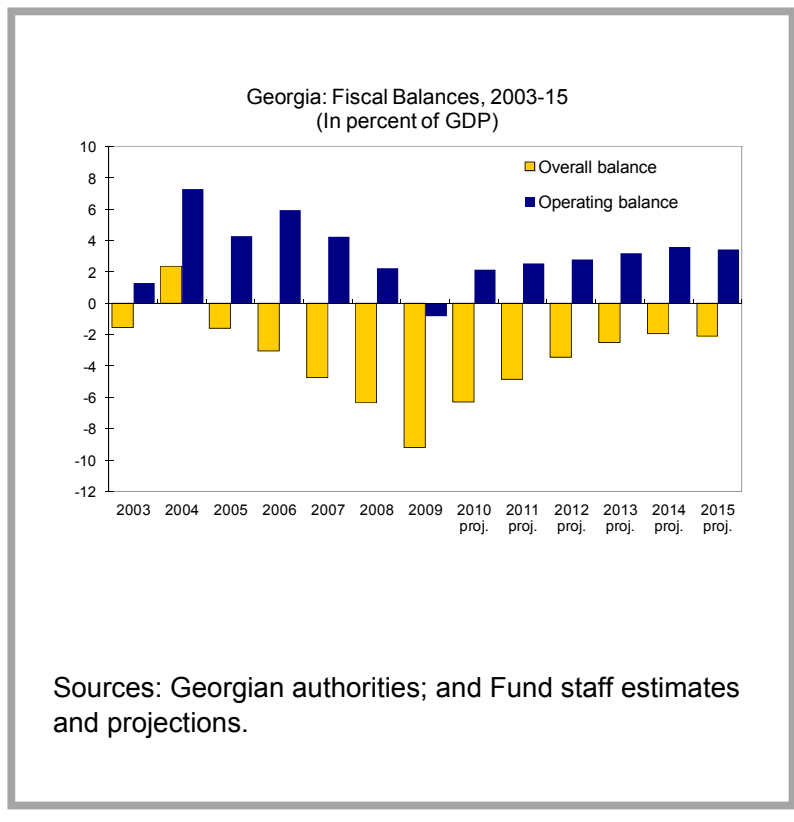
disasters (earthquake and flooding), housing for internally displaced persons, and capital projects including new customs clearance and border checking infrastructure to facilitate trade, and initial work on the relocation of the parliament to Kutaisi (the second largest city).

Georgia: Structural Deficit and Fiscal Impulse, 2009-10

(In percent of GDP)

\begin{tabular}{lccc}
\hline & 2009 & \multicolumn{2}{c}{2010} \\
\cline { 2 - 4 } & & 5th Rev. & 6th Rev. \\
\hline Overall deficit & -9.2 & -7.4 & -6.3 \\
Structural deficit 1/ & -6.2 & -3.3 & -3.6 \\
Fiscal impulse 1/ & 4.0 & -1.1 & -1.8 \\
\hline
\end{tabular}

Sources: Ministry of Finance; and Fund staff estimates.

$1 /$ The calculation of the two indicators is described in Box 2 of EBS/09/188.

\section{In view of exchange rate pressures experienced in May and the need for faster} external adjustment, the authorities decided in June to cut back on the proposed spending increase and to introduce new revenue measures in 2010-11. The net impact of these adjustment measures on the 2010 budget is estimated at 0.5 percent of GDP: 0.2 percent of GDP from scaling back the supplementary budget spending request, and 0.3 percent of GDP from new excises coming into effect on August 1, 2010. Additional 
revenue measures equivalent to 0.9 percent of GDP will come into effect on January 1, 2011, including harmonization of the income tax rate and expansion of the VAT base (LOI, ๆ19). ${ }^{1}$ Parliament is expected to vote on these revenue measures in July 2010, as part of the new tax and customs code, which also defers to (no earlier than) 2013 the planned reduction of the personal income tax rate from 20 to 15 percent. $^{2}$ The scaled-back supplementary budget spending request was submitted to parliament on June 4 and is expected to be approved by end-June.

\section{In all, the $\mathbf{2 0 1 0}$ fiscal deficit target is reduced from 7.4 percent of GDP (fifth} review) to 6.3 percent, resulting in a nearly 3 points of GDP decline relative to 2009 . The structural deficit is marginally higher than envisaged at the time of the fifth review on account of the additional spending, but the withdrawal of fiscal stimulus, as measured by the fiscal impulse, is much larger. ${ }^{3}$ The authorities will lock in the new level of spending through an explicit ceiling, so that any additional revenue would be directed entirely to faster deficit reduction (LOI, $\mid 19)$.

\section{While restating their commitment to expenditure-based adjustment over the} medium term, the authorities also plan to increase tax policy flexibility by postponing implementation of the proposed referendum requirement for new taxes. This proposed constitutional amendment will now become effective only once the deficit has been reduced to below 3 percent of GDP (LOI, $\mid 18$ ). No action has been taken to advance the proposed Economic Freedom, Opportunity, and Dignity Act: the draft document, which would enshrine liberal economic principles and set limits on government spending, deficit, and debt, was to be considered as a separate organic law, but has not yet been formally presented to parliament. $^{4}$

17. With additional long-term official budgetary support becoming available, the authorities will reduce the share of Fund financing that is channeled through the budget in 2010. ${ }^{5}$ The tranche that would become available upon completion of the sixth

\footnotetext{
${ }^{1}$ In particular, the package includes: elimination of the reduced personal income tax rate; enlargement of the capital gain tax base; increase in excises on beer, spirit, and scrap metal; introduction of an excise on telecommunication services; and elimination of VAT exemptions for private education and imported medical equipment.

${ }^{2}$ The amended tax code will also remove the planned reduction of the tax on interest and dividends, which remains at 5 percent.

${ }^{3}$ The fiscal impulse includes all changes in tax revenues, including the cyclical component. This is the main reason for the negative value in 2010 .

${ }^{4}$ The proposed Act is described in Box 2 of Country Report No. 09/331.

${ }^{5}$ The overall balance of payment need covered by Fund financing in 2010 remains unchanged at US\$446 million.
} 
review would be the last one to be channeled through the budget in 2010 (LOI, ๆ29). The government has tapped successfully into the domestic market, and the maturity structure has been lengthened with issuance of one year and two year paper in order to develop the local securities market. With T-bill rates on the rise, the authorities do not see scope for increasing domestic financing much beyond the current level without the risk of crowding out private sector credit.

Georgia: External Lending and Grants to the Public Sector, 2008-10 (Gross inflows, in millions of U.S. dollars)

\begin{tabular}{lrrrr}
\hline & 2008 & 2009 & 2010 \\
\cline { 4 - 5 } & & \multicolumn{2}{c}{$5^{\text {th }}$ Rev. } & Proj. \\
\hline Total & 1368 & 1025 & 1082 & 1174 \\
$\quad$ IMF 1/ & 257 & 340 & 470 & 446 \\
$\quad$ Of which: through the budget & 0 & 102 & 390 & 298 \\
$\quad$ WB & 126 & 274 & 191 & 189 \\
$\quad$ Other multilateral institutions & 135 & 237 & 189 & 287 \\
$\quad$ Bilateral partners & 350 & 172 & 231 & 252 \\
$\quad$ Eurobond-2013 & 500 & $\ldots$ & $\ldots$ & $\ldots$ \\
& & & & \\
Memorandum items: & & & & \\
Total excluding IMF & 1111 & 685 & 613 & 728 \\
by type: & & & & \\
$\quad$ Grants & 388 & 259 & 282 & 324 \\
$\quad$ Loans 2/ 3/ & 723 & 426 & 331 & 404 \\
by purpose: & & & & \\
$\quad$ Budget support financing 3/ & 426 & 273 & 161 & 220 \\
$\quad$ Project financing & 185 & 412 & 452 & 508 \\
\hline
\end{tabular}

Source: Georgian authorities; and Fund staff estimates.

$1 /$ Includes augmentation of access in 2010-11.

2/ Includes Eurobond in 2008.

3/ Excludes SBA purchases. 
Georgia: Fiscal Adjustment, 2009-14

\begin{tabular}{|c|c|c|c|}
\hline Measures & $\begin{array}{l}\text { Change } \\
2009-10\end{array}$ & $\begin{array}{l}\text { Change } \\
2010-14\end{array}$ & Comments \\
\hline & (In perce & ent of GDP) & \\
\hline (1) Policy adjustment and automatic stabilizers & 2.5 & 7.1 & \\
\hline Increase in revenues & 0.4 & 0.7 & \\
\hline Tax revenues $1 /$ & 0.8 & 1.1 & $\begin{array}{l}\text { New and higher excises, small adjustment to the customs tariff structure, reappraisal of property, improvement in revenue collection (2010), harmonization of personal } \\
\text { income tax rates, VAT base expansion (2011) }\end{array}$ \\
\hline Other revenues & -0.5 & -0.4 & $\begin{array}{l}\text { Impact of } 2009 \text { activity slowdown on dividends from public enterprises and interest from net lending (2010), lower dividends due to privatization and retained earnings to } \\
\text { finance infrastructure projects (2011-2014) }\end{array}$ \\
\hline Decrease in expenditures & 2.1 & 6.4 & \\
\hline Current spending $2 /$ & 2.2 & 3.5 & \\
\hline Compensation for employees & 0.0 & 0.9 & $\begin{array}{l}\text { Wage bill containment (defense personnel, bonus cuts, limits on number of contractual employees) offset by reclassification of legal entities of public law (LEPL) } \\
\text { expenditures (2010), constant real wage bill (2011-2013) }\end{array}$ \\
\hline Use of goods and services & 1.0 & 0.5 & $\begin{array}{l}\text { Cuts in administrative expenses of } 10-15 \% \text { in all ministries in nominal terms (2010), and of } 4 \% \text { cumulatively in real terms, except in ministries of education, health and } \\
\text { agriculture (2010-2014). }\end{array}$ \\
\hline Subsidies & 0.4 & 0.7 & Reclassification of LEPL expenditures from subsidies to other categories (2010), and cuts of $23 \%$ cumulatively in real terms (2010-2014) \\
\hline Social expenses & 0.2 & 0.4 & $\begin{array}{l}\text { Increase by } 4 \% \text { in real term in } 2010 \text { (pensions, social assistance, health reform 3/), efficiency gains and decrease in number of beneficiaries of subsistence minimum (2011- } \\
\text { 2014) }\end{array}$ \\
\hline Other expenses & 0.7 & 1.0 & Decrease in reserve funds (2010) and cofinancing of investment projects (2010-2014), phasing out of house construction for IDPs (from 2011 on) \\
\hline Capital spending & -0.6 & 3.1 & $\begin{array}{l}\text { Budget supplement (2010), Completion of main city rehabilitation and infrastructure projects financed by donors; less reconstruction/renovation of public buildings (2011- } \\
\text { 2014) }\end{array}$ \\
\hline Net lending & 0.5 & -0.2 & Subsidized lending program (2009) and related repayments (2010) \\
\hline (2) Exogenous factors & 0.4 & -2.7 & \\
\hline Increase in revenues & 0.1 & -2.6 & \\
\hline Tax arrears clearance & -0.6 & -0.2 & Belated VAT payment (2009) and penalty (2010) received from the Ministry of Defense \\
\hline External grants & 0.7 & -2.4 & Reduction in donor support (2010-2014) \\
\hline Decrease in expenditures & 0.2 & -0.1 & \\
\hline Tax arrears clearance & 0.6 & 0.2 & Belated VAT payment (2009) and penalty (2010) paid by the Ministry of Defense \\
\hline Interest payments & -0.3 & -0.3 & Public debt increase (2010-2014) \\
\hline$(1)+(2)=$ Total reduction in fiscal deficit & 2.9 & 4.4 & \\
\hline
\end{tabular}

Sources: Ministry of Finance; and Fund staff estimates.

Note: Figures may not add up due to rounding.

1/ Excluding intra-government tax arrears clearance.

2/ Excluding interest payments and intra-government tax arrears clearance.

$3 /$ Shift to insurance system and increase in the number of beneficiaries. 
18. The external public debt-to-GDP ratio would peak at 43.2 percent of GDP in 2011, declining thereafter to 32.7 percent by 2015. Vulnerabilities associated with this level of debt warrant continued monitoring. However, the indicative ceiling on nonconcessional public external borrowing no longer appears justified given the authorities' strong debt management capacity, their fiscal consolidation plans, and their intention to return to market financing as soon as conditions permit. Accordingly, it is proposed that the indicative ceiling be set on total public external borrowing.

\section{The medium-term fiscal framework} will be adopted by the government in July 2010 (structural benchmark, LOI, $\uparrow 21$ ). The authorities are committed to reducing the deficit to 2-3 percent of GDP by 2013 (LOI, $₫ 18$ ). Current deficit projections imply a contraction of real spending of 3 percent in 2011 relative to 2010. Thereafter, expenditure contraction eases, but government spending in relation to GDP falls by another 4 percentage points by 2014 .

\section{The authorities plan to move to a full} program presentation of the budget by 2012 . Based on technical assistance provided by the IMF's Fiscal Affairs Department, they intend to adopt guidelines for line ministries by endSeptember (structural benchmark, LOI, $\mid 21$ ).

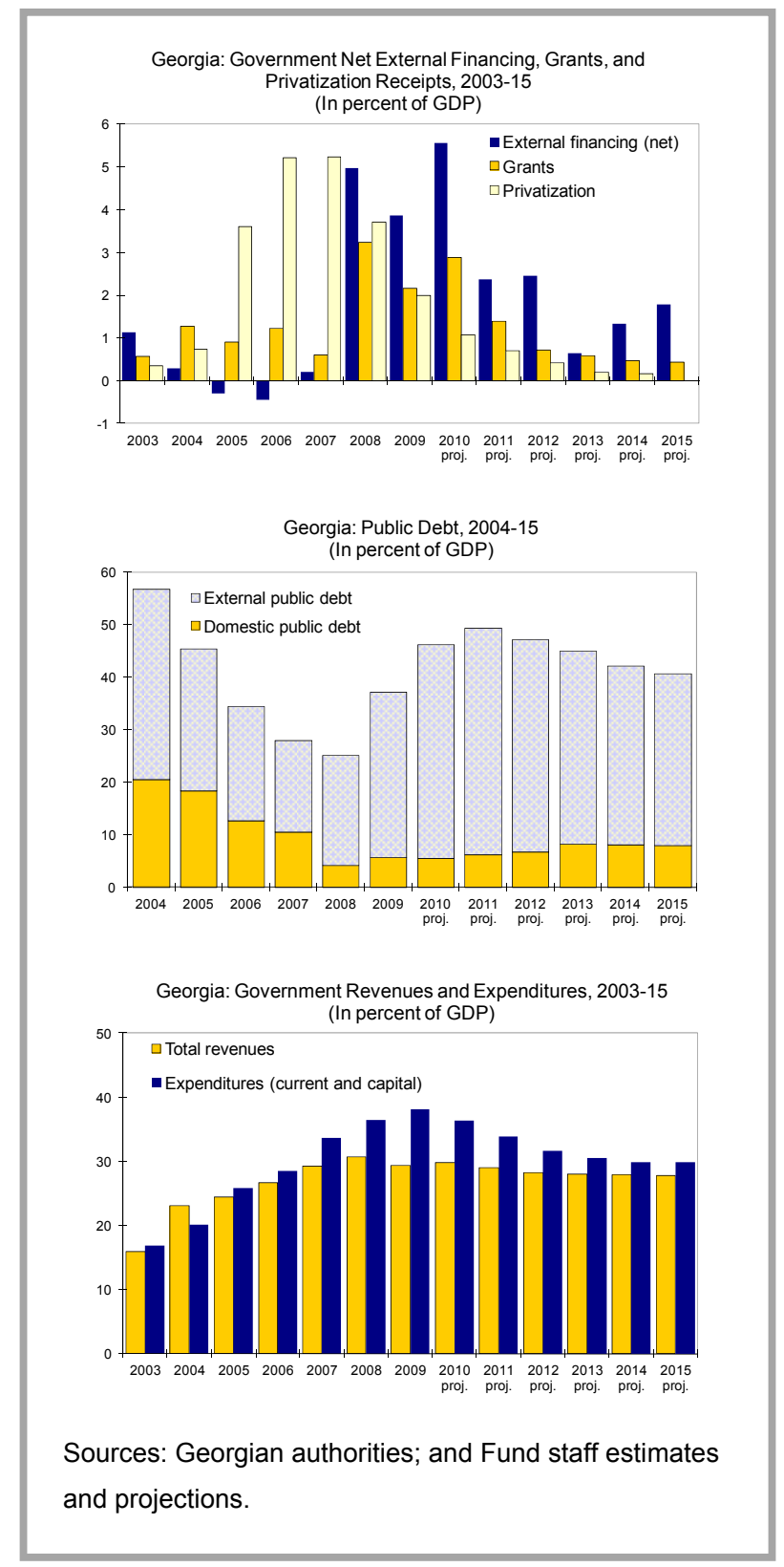

\section{Monetary Policy and Credit Conditions}

21. Monetary policy tightening began in June in reaction to unstable exchange rate market conditions and related concerns about inflation, and has already been reflected in market rates. Steady deposit growth through May and measures taken to improve access to the central bank's refinancing window (Box 1) reduced banks' demand for precautionary balances, resulting in an easing of credit conditions and higher local currency lending. Central bank refinancing also increased by the equivalent of 9 percent of reserve money in April-May. As part of the package of corrective measures taken to reduce pressures in the foreign exchange market, the refinancing rate was raised from 5 to $6 \frac{1 / 4}{4}$ percent on June 16 . The authorities pointed out that market rates (T-bill, CD, and interbank rates) had already begun rising in anticipation of monetary tightening, as evidenced by the upward shift of the 
yield curve. Considerable dedollarization took place in the first four months of the year. The faster depreciation experienced in June did not trigger any large scale movements out of local currency deposits.
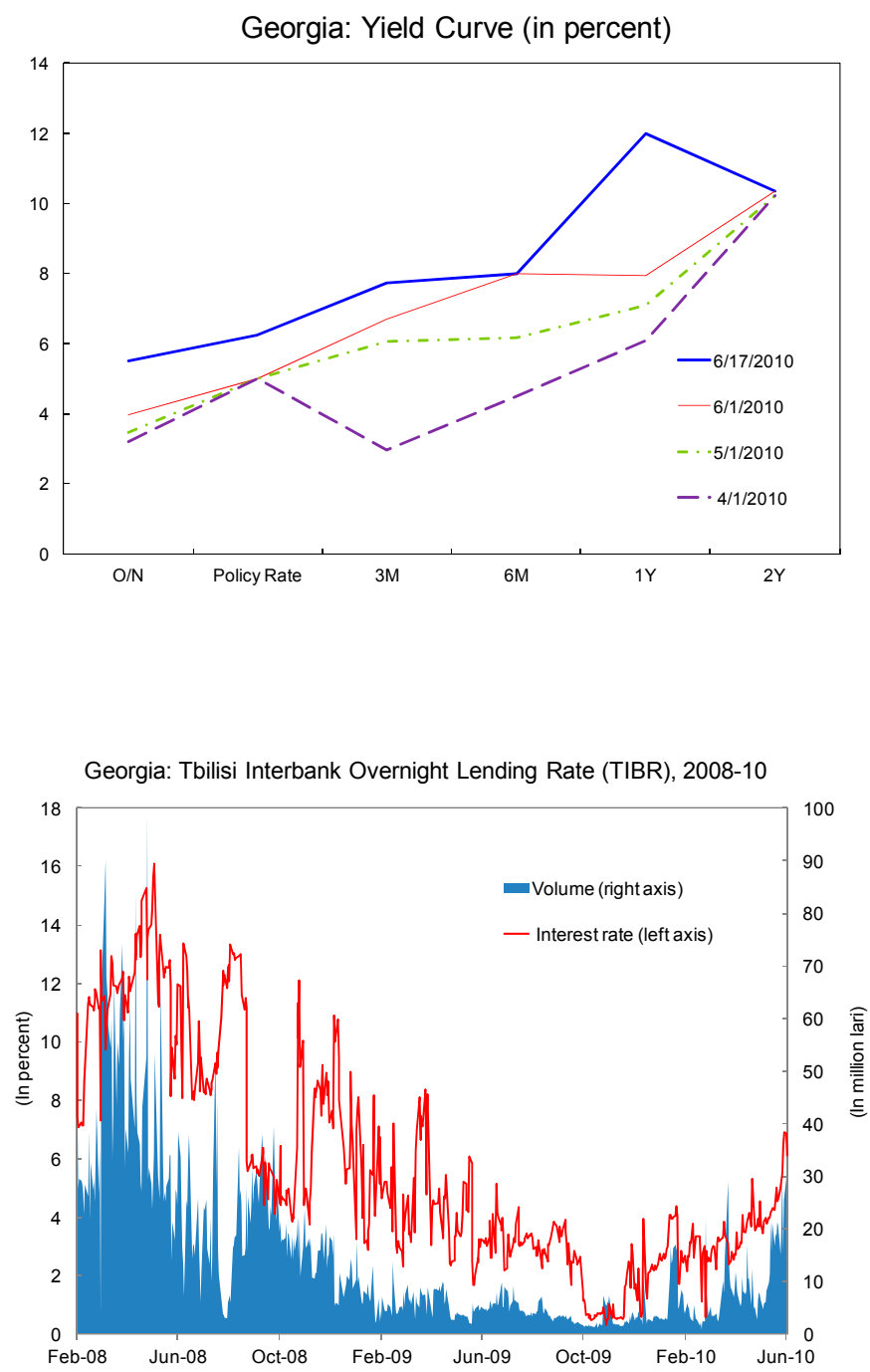

Sources: Georgian authorities; and Fund staff estimates. 


\section{Box 1. Georgia: Measures to Enhance Monetary Policy Effectiveness and Encourage Financial Dedollarization}

The following measures became effective April 29:

- The reserve requirement on lari-denominated deposits is raised from 5 to 10 percent to reduce free reserves and give the central bank's loan facilities more traction. Required reserve in excess of 5 percent will be remunerated at the policy rate to avoid distortions and lari disintermediation. The reserve requirement on foreign exchange deposits is maintained at 5 percent for the time being.

- External borrowing will be subject to reserve requirements starting in September 2010, at the same rate that applies to domestic foreign exchange funding sources.

- Banks are given access to a new standing facility in the form of overnight deposits remunerated at the policy rate minus 200 basis points.

- Central bank loan facilities are enhanced by guaranteeing access to a new 7-day collateralized refinancing facility at a rate equal to the policy rate plus 100 basis points. Access to this facility is limited to GEL 200 million per week. Banks' individual limits are based on capital. In addition, access to overnight loans is simplified, and the interest rate set equal to the policy rate plus 200 basis points. These two facilities provide a liquidity safety net to banks, should they fail to secure liquidity through the regular weekly auctions. The policy rate sets a floor for the weekly liquidity auctions.

- Eligible collateral in operations with the central bank is expanded to include laridenominated loans of six-month or longer maturity and guarantees provided by IFIs and international commercial banks meeting minimum criteria. Collateralized loans are subject to a haircut and have to satisfy credit risk criteria set by the National Bank of Georgia (NBG). Foreign exchange is no longer allowed as collateral in any operations with NBG.

22. The monetary program is built on an expected continued growth in deposits, but a slight deceleration of credit growth in the second half of the year, in line with recent monetary tightening and possible increase in NPLs due to exchange rate depreciation. Projected annual growth in credit to the economy (adjusted for valuation changes) has been revised up relative to the fifth review, from 6 to $9 \frac{1}{2}$ percent. The revision reflects the stronger growth outlook and improved liquidity conditions of banks. Demand for free reserves is expected to decline reflecting enhanced access to central bank refinancing and greater stability of the deposit base. On that basis, the NDA targets for September have been revised down but the end-December ceiling has been raised (relative to the indicative target) on account of higher estimated demand for currency in circulation. 
23. Recent exchange rate depreciation will adversely affect banks' balance sheets, but capital buffers (17.6 percent capital adequacy in May) should be sufficient to absorb the impact for most banks. Stress tests conducted by the National Bank suggest that a one-time 15 percent depreciation would, within 1 year, result in a 5.3 point decline in the banking system's capital adequacy ratio. Of that, 3.8 points would come from higher provisioning requirements due to the deterioration of the dollar loan portfolio; the rest from valuation effects. ${ }^{6}$

\section{Medium-Term Outlook and the Exit Strategy}

24. The current exit strategy from official balance of payments support is predicated on a steady increase in private capital inflows (mainly FDI), which by 2012 should enable Georgia to return to a path of privately financed high economic growth. Starting in 2012, external financing needs would be covered entirely by private sector inflows and regular official financing. After stalling in the first half of 2010, FDI is projected to recover in the second half of 2010 to the level of the same period of 2009, and to increase moderately in 2011 . The projected increase in FDI over the medium term is within the range projected for other emerging market economies. In the pre-crisis years, FDI in Georgia was boosted by privatization, which attracted FDI of about 21/2 percent of GDP on average in 2006-07. Even with the privatization program winding down, Georgia is well positioned to attract significant green-

\footnotetext{
${ }^{6}$ The analysis is based on a 5 percent elasticity of NPLs to exchange rate change, which implies an increase of NPLs from 18 percent to 27 percent of the loan portfolio. This elasticity is higher than the elasticity (3.6) which was estimated based on Georgian data with the help of IMF technical assistance in April 2009. The assumed higher elasticity reflects the expectation of a non-linear response of NPLs to large exchange rate changes, such as the one assumed in this exercise. It is also likely to constitute an upper bound. Valuation effects arise because capital and provisions (even against dollar loans) are denominated in local currency.
}

Sources: National Bank of Georgia, Bloomberg, World Economic Outlook, and Fund staff estimates.
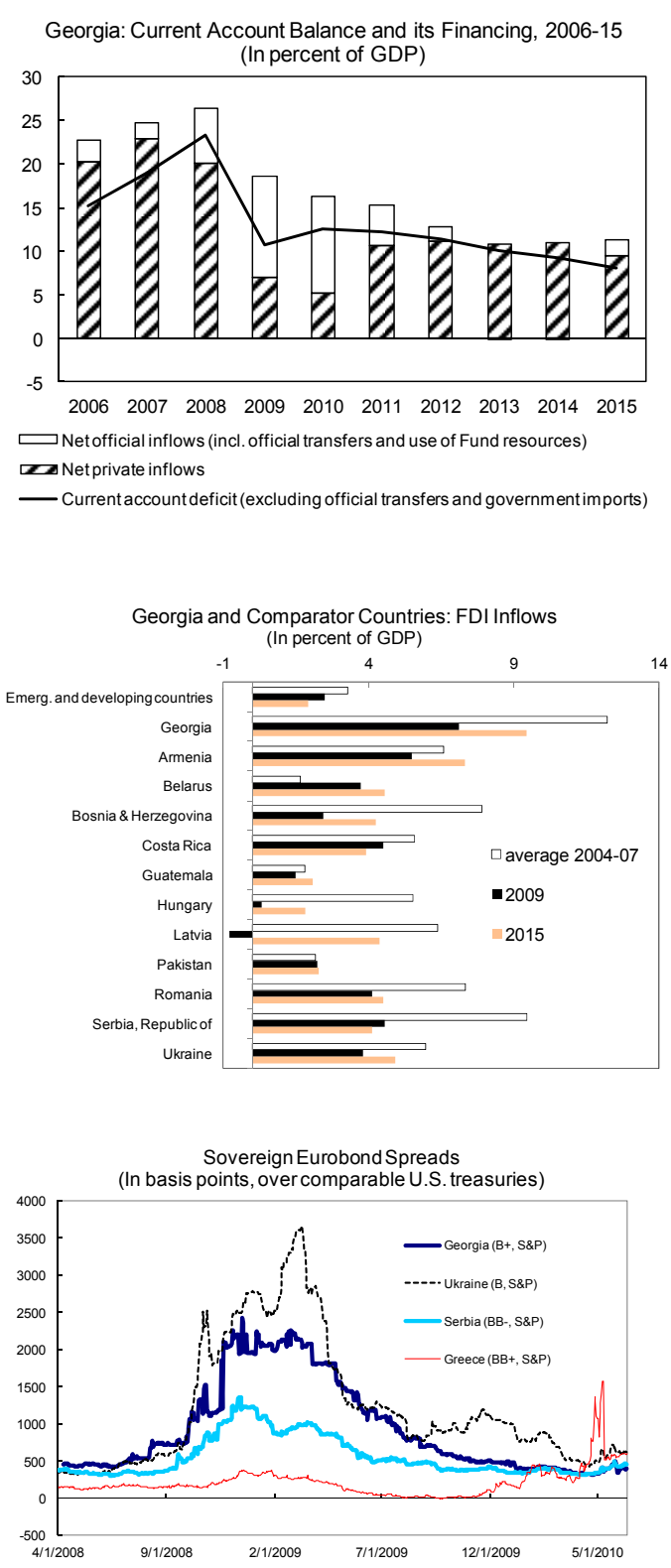

\section{d}


field FDI, capitalizing on the strength of its business climate, the government's reformist agenda, and its geographical location linking Europe to Central Asia.

\section{The authorities have identified contingency measures they would take in the} event significant balance of payments pressures reemerged this year. While continuing to rely on exchange rate adjustment, the authorities would first accelerate the pace of monetary tightening and deficit reduction in order to contain the impact of depreciation on the balance sheet of banks (LOI, ๆ26).

\section{Projected private capital inflows and current account deficits over the medium} term have been lowered since the fifth review. This reflects a more conservative outlook on the availability of external private financing, and, on the current account side, a combination of more favorable terms of trade and somewhat lower import growth due to lower FDI.

\section{The authorities expect to meet the large debt service peak of 2013-14 by} accessing capital markets as soon as conditions permit. Georgia's Eurobond spreads have been affected in line with those of other emerging market countries by instability in the euro zone. However, market access has been enhanced by an upgraded rating from S\&P in April 2010.

Georgia: Ranking Based on Selected Policy Performance Indicators, 2009-10

\begin{tabular}{|l|c|c|c|c|}
\hline & Coverage & Rank overall & $\begin{array}{c}\text { Rank in Eastern Europe } \\
\text { and Central Asia }\end{array}$ & $\begin{array}{c}\text { Peers (same score } \\
\text { or ranking) }\end{array}$ \\
\hline $\begin{array}{l}\text { World Bank Ease of Doing } \\
\text { Business Index, 2010 }\end{array}$ & 183 countries & $11^{\text {th }}$ & $1^{\text {st }}$ out of 27 & $\begin{array}{c}\text { between Norway } \\
\text { and Thailand }\end{array}$ \\
\hline $\begin{array}{l}\text { World Bank Country Policy } \\
\text { and Institutional } \\
\text { Assessment, 2010 1/ }\end{array}$ & $\begin{array}{c}77 \text { IDA-eligible } \\
\text { countries }\end{array}$ & $1^{\text {st }}$ & $1^{\text {st }}$ out of 9 & $\begin{array}{c}\text { Just ahead of } \\
\text { Armenia, Cape } \\
\text { Verde, Uganda, } \\
\text { and Ghana }\end{array}$ \\
\hline $\begin{array}{l}\text { Transparency International } \\
\text { Corruption Perception } \\
\text { Index, 2009 }\end{array}$ & 180 countries & $66^{\text {th }}$ & $2^{\text {nd }}$ out of 20 & Croatia, Kuwait \\
\hline
\end{tabular}

Sources: Transparency International; and the World Bank.

1/ Based on assessment of economic management, structural policies, policies for social inclusion/equity, and public sector management and institutions.

\section{E. Risks to the Medium-Term Strategy}

- FDI. A failure of FDI to rebound would adversely affect GDP growth but, because imports would also decline, the resulting external imbalance would be to a large extent self correcting. However, the growth shortfall would widen the fiscal deficit, requiring additional adjustment to preserve debt sustainability (Box 2). 
- Debt Rollover. The Debt Sustainability Analysis (DSA) points to low solvency risks, but relatively high rollover risks in 2013 linked to the cumulation of Fund and Eurobond repayment obligations.

- Banking Sector. Banking sector risks are tied to the high level of NPLs and the exposure to currency-induced credit risks. Given the solid capitalization, provisioning and liquidity buffers, banking sector vulnerabilities are unlikely to become a crisis trigger. However, they could become a destabilizing factor in the event of another shock to economic activity or the exchange rate.

\section{Box 2. Georgia: Alternative Scenario with Lower FDI}

Reliance on a rebound of FDI to finance investment and to fill the current account deficit exposes the economy to external risks. These risks are explored in an alternative scenario that assumes a stabilization of FDI at its 2010 level (61/2 percent of GDP). Assuming an unchanged productivity of capital, the drop in overall investment would reduce real GDP growth to $3 \frac{1}{2}$ percent on average over the next 5 years.

Even with an assumed loss of export competitiveness, lower investment and real GDP growth would compress the current account deficit quite sizably. The balance of payments gap (relative to the baseline scenario) would be equivalent to 0.8 percent of GDP on average over the period 2011-15, rising to 1.5 percent of GDP by 2015 , and would cause a loss of international reserves of US\$466 million by 2015 .

An imbalance would also appear in the fiscal accounts. Based on the same fiscal policy of the baseline scenario (same level of real expenditure and same tax-to-GDP ratio), the deficit would begin to rise again and reach 4.3 percent of GDP by 2015 . The primary fiscal balance would exceed the debtstabilizing level by 0.6 percentage point of GDP.

\begin{tabular}{|c|c|c|c|c|c|c|}
\hline \multicolumn{7}{|c|}{ Georgia: Alternative Medium-Term Scenarios (2011-15) } \\
\hline & \multicolumn{3}{|c|}{$2011-15$ average } & \multicolumn{3}{|c|}{2015} \\
\hline & 5th Review & baseline & $\begin{array}{c}\text { low } \\
\text { growth }\end{array}$ & 5th Review & baseline & $\begin{array}{c}\text { low } \\
\text { growth }\end{array}$ \\
\hline Real GDP growth & 4.8 & 4.8 & 3.5 & 5.0 & 5.0 & 3.5 \\
\hline Investment rate (in percent of GDP) & 20.0 & 19.0 & 13.4 & 23.0 & 20.0 & 13.3 \\
\hline ICOR (change in capital stock / real GDP growth) & 4.2 & 4.0 & 3.8 & 4.6 & 4.0 & 3.8 \\
\hline \multicolumn{7}{|l|}{ General Government (in percent of GDP) } \\
\hline Revenues & 27.0 & 28.2 & 28.2 & 26.8 & 27.8 & 27.9 \\
\hline Tax revenue & 24.3 & 25.6 & 25.6 & 24.3 & 25.6 & 25.6 \\
\hline Current expenditure & 24.2 & 25.1 & 26.1 & 23.3 & 24.4 & 26.2 \\
\hline Capital expenditure & 5.8 & 6.1 & 6.3 & 5.2 & 5.6 & 5.9 \\
\hline Balance & -3.0 & -3.0 & -4.1 & -1.7 & -2.1 & -4.3 \\
\hline Total Public Debt & 45.2 & 44.8 & 48.0 & 40.8 & 40.5 & 47.2 \\
\hline \multicolumn{7}{|l|}{ Investment and saving (in percent of GDP) } \\
\hline Investment $1 /$ & 20.0 & 19.0 & 13.4 & 23.0 & 20.0 & 13.3 \\
\hline Public & 5.6 & 5.8 & 6.0 & 5.2 & 5.6 & 5.9 \\
\hline Private & 14.4 & 13.3 & 7.4 & 17.8 & 14.4 & 7.4 \\
\hline Gross national saving & 6.8 & 7.5 & 3.5 & 10.3 & 10.4 & 5.1 \\
\hline Public & 2.8 & 3.1 & 2.2 & 3.5 & 3.5 & 1.6 \\
\hline Private & 4.0 & 4.4 & 1.3 & 6.8 & 6.9 & 3.5 \\
\hline Saving-investment balance $=$ current account balance & -13.2 & -11.5 & -9.9 & -12.7 & -9.6 & -8.2 \\
\hline \multicolumn{7}{|l|}{ External sector } \\
\hline Exports of goods and services (percent of GDP) & 34.8 & 39.7 & 39.8 & 35.3 & 40.2 & 39.2 \\
\hline volume, percentage change & 8.2 & 8.5 & 6.4 & 6.6 & 8.2 & 3.8 \\
\hline percent change in volume/percent real GDP growth $2 /$ & 1.7 & 1.8 & 1.8 & $\ldots$ & $\ldots$ & \\
\hline Imports of goods and services (percent of GDP) & 53.9 & 56.7 & 55.5 & 53.3 & 54.7 & 52.8 \\
\hline volume, percentage change & 5.9 & 5.2 & 2.9 & 6.0 & 5.1 & 3.1 \\
\hline percent change in volume/ percent real GDP growth $2 /$ & 1.2 & 1.1 & 0.8 & $\ldots$ & & \\
\hline Gross international reserves (in millions of US $\$$ ) & 2,414 & 2,175 & 1,967 & 2,551 & 2,221 & 1,755 \\
\hline Gross international reserves (in percent of total external debt) & 32 & 26 & 24 & 32 & 26 & 20 \\
\hline Foreign direct investment (percent of GDP) & 11.4 & 8.9 & 6.5 & 12.3 & 9.4 & 6.5 \\
\hline
\end{tabular}



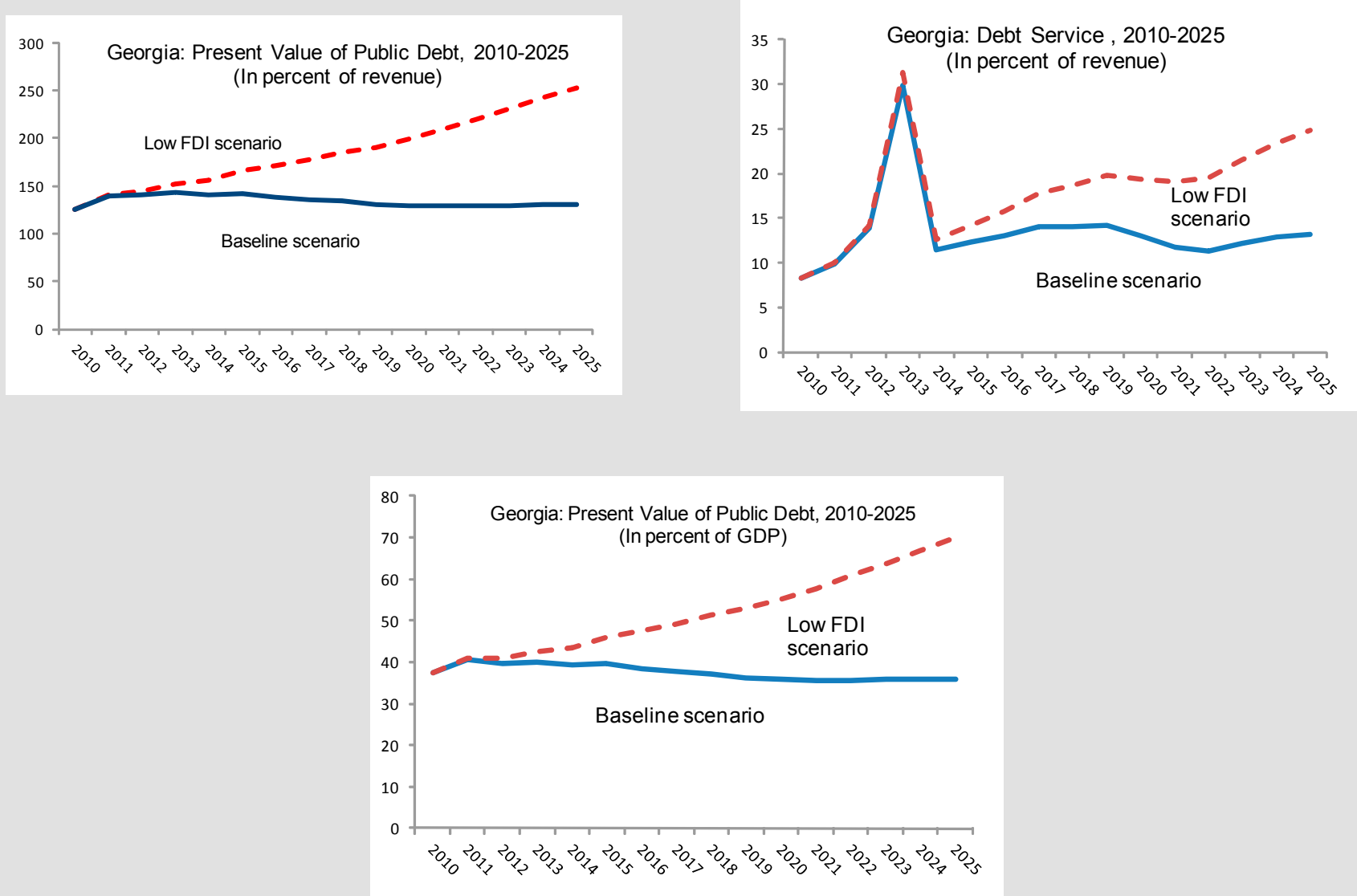

Sources: Georgian authorities; and Fund staff estimates and projections.

\section{Program Issues}

28. Modifications to the performance criteria for end-September 2010, and establishment of new performance criteria for end-September and end-December 2010 are being proposed as follows (LOI, Table 1):

- NIR: the floors of end-September and end-December (relative to the indicative target) have been raised to reflect higher projected external inflows which outweigh the impact of higher intervention.

- NDA: the end-September ceiling has been lowered on account of revised demand for free reserves, but the end-December ceiling has been raised (relative to the indicative target) on account of higher estimated demand for currency in circulation.

- Budget deficit: the end-September ceiling has been lowered in line with the revised annual deficit target which is reflected in the end-December ceiling (also lower than the previous indicative target). Furthermore, the definition has been modified to cover 
the general government rather than the consolidated government, which is the measure that is de facto available.

- Government spending (new performance criteria): ceilings have been set for September and December 2010, in line with the government's supplementary budget request.

29. The end-June NDA performance criterion is expected to be met, but its status will be reported separately on July 7 .

30. The following waivers are requested for end-June performance criteria:

- Waiver of nonobservance of performance criterion on NIR, justified by the corrective measures taken to address the underlying imbalance, as described above in qฯ 2, 9, 14 and 21 . The precise status of the performance criterion will be reported separately on July 7.

- Waiver of applicability of performance criterion on the fiscal deficit, based on the fact that preliminary information through May 2010 indicates that the target will be met, but that the data to verify observance will not become available before July 27 .

31. Rephasing of purchases by combining the seventh and eight reviews is requested due to the delay in completing the sixth review and the objective of meeting the balance of payments financing needs in 2010.

\begin{tabular}{|c|c|c|}
\hline \multicolumn{3}{|c|}{ Georgia: Structural Benchmarks for 2010} \\
\hline Structural Benchmark & Time Frame & Rationale \\
\hline $\begin{array}{l}\text { Cabinet approval of a new medium-term expenditure } \\
\text { framework (Basic Data and Directions) that includes } \\
\text { expenditure ceilings applicable to the } 2011 \text { budget. }\end{array}$ & July 31, 2010 & \multirow{2}{*}{$\begin{array}{l}\text { Strengthen the efficiency of the } \\
\text { adjustment strategy by improving } \\
\text { linkages between the medium- } \\
\text { term expenditure framework and } \\
\text { annual budgets, and building the } \\
\text { capacity for program budgeting. }\end{array}$} \\
\hline $\begin{array}{l}\text { Cabinet approval of guidelines for pilot Ministries to } \\
\text { introduce a programmatic approach to budgeting, in line } \\
\text { with the recommendations of the IMF technical assistance } \\
\text { report "Georgia-Advancing Program Budgeting," by D. } \\
\text { Radev, S. Flynn, L. Eyraud, and S. Gurr, May } 2010 \text {. }\end{array}$ & September 30, 2010 & \\
\hline
\end{tabular}

\section{Staff Appraisal}

32. The economic recovery has gained strength. Recent global financial market turbulence has shifted the balance of risks in 2010 from domestic to external factors. On the domestic front, the orderly completion of municipal elections and a resumption of credit growth should help sustain the growth momentum.

33. The corrective policy actions taken in June to ensure faster external adjustment provide adequate assurances that the program objectives are on track. Persistent exchange rate pressures in April-May were symptoms of a larger-than-expected structural 
imbalance (beyond seasonal factors), due to weakness in private capital inflows compounded by relatively expansionary fiscal and monetary policies in the second quarter. Delayed exchange rate adjustment has resulted in the likely nonobservance of the end-June NIR target. However, faster depreciation starting in June, and the tightening of monetary and fiscal policies should address this imbalance. Going forward, exchange rate flexibility remains key to ensuring that the economic recovery is externally sustainable. The constraint placed by the NIR targets on intervention provides assurances against inadequate exchange rate adjustment in the face of new external imbalances.

34. The sizeable reduction in the fiscal deficit targeted for $\mathbf{2 0 1 0}$ under the program - by nearly 3 percentage points of GDP_brings the medium-term consolidation target within closer reach. Because of the stronger cyclical rebound, the deficit is considerably lower in terms of GDP than initially estimated, even with the higher spending under the supplementary budget. The adoption of additional revenue measures, and the authorities' commitment to capping spending in 2010 render fiscal policy appropriately countercyclical going forward.

35. The authorities' success in mobilizing additional budgetary support and diversifying their financing sources in $\mathbf{2 0 1 0}$ is welcome. By allowing to reduce correspondingly the amount of Fund financing channeled through the budget, new external long-term financing helps smooth the government's debt rollover hump of 2013-14. Steady issuance of T-Bills and the extension of maturities create the foundations for capital market development, with benefits for local currency funding and monetary policy.

36. The decision to postpone implementation of a referendum requirement on tax increases until the fiscal deficit has been returned to prudent levels enhances policy flexibility. Equally welcome is the introduction of new revenue measures in 2010 and 2011. Without undermining the authorities' emphasis on expenditure containment as the primary means of adjustment, these initiatives strengthen the credibility of the adjustment strategy.

\section{The recent tightening of monetary policy was warranted and should continue.} Enhanced access to the central bank's refinancing facilities requires that policy interest rates be adjusted promptly and flexibly to changing conditions, including foreign exchange market pressures or signs of accelerating monetary and credit growth. Higher interest rates would also encourage deposit dedollarization.

38. The balance sheet impact of recent depreciation calls for continued close monitoring of the banking sector. The ongoing economic recovery should limit the adverse real sector impact of rising NPLs, and timely corrective actions can be expected given the close supervision of banks.

39. The exit strategy has been reinforced by improvements in confidence accompanying the rebound in activity, which place Georgia in a good position to reaccess financial markets ahead of the rollover needs of 2013-14. The program is now built on an assumed slower recovery of FDI. While risks remain, they appear to be 
manageable within the current policy framework. However, a failure of FDI inflows to materialize in the second half of the year would signal the need for much more fundamental policy changes. The authorities' contingency plans provide assurances that a timely response to such risks can be activated. Beyond 2010, the authorities are encouraged to create stronger buffers against downside risks by targeting an ambitious reduction in the fiscal deficit in 2011, ahead of the 2012 and 2013 election years.

40. On the basis of Georgia's performance under the SBA and the corrective actions taken to address the causes of the nonobservance of the end-June NIR target, staff supports the authorities' request for completing the sixth review, modifying and establishing performance criteria, and rephasing purchases by combining the seventh and the eighth reviews as specified in the LOI ( $₫ 32$ and Table 1). 
Table 1. Georgia: Selected Macroeconomic Indicators, 2008-15

\begin{tabular}{|c|c|c|c|c|c|c|c|c|}
\hline & $\begin{array}{r}2008 \\
\text { Act. }\end{array}$ & $\begin{array}{r}2009 \\
\text { Prel. }\end{array}$ & $\begin{array}{r}2010 \\
\text { Proj. }\end{array}$ & $\begin{array}{r}2011 \\
\text { Proj. }\end{array}$ & $\begin{array}{r}2012 \\
\text { Proj. }\end{array}$ & $\begin{array}{r}2013 \\
\text { Proj. }\end{array}$ & $\begin{array}{r}2014 \\
\text { Proj. }\end{array}$ & $\begin{array}{r}2015 \\
\text { Proj. }\end{array}$ \\
\hline & \multicolumn{8}{|c|}{ (Annual percentage change, unless otherwise indicated) } \\
\hline \multicolumn{9}{|l|}{ National accounts } \\
\hline Nominal GDP (in million lari) & 19,075 & 17,949 & 19,657 & 21,465 & 23,665 & 26,091 & 28,765 & 31,714 \\
\hline Real GDP growth & 2.3 & -3.9 & 4.5 & 4.0 & 5.0 & 5.0 & 5.0 & 5.0 \\
\hline Population (in million) 1/ & 4.4 & 4.4 & 4.4 & 4.4 & 4.4 & 4.4 & 4.4 & 4.4 \\
\hline GDP deflator, period average & 9.7 & -2.0 & 4.8 & 5.0 & 5.0 & 5.0 & 5.0 & 5.0 \\
\hline Consumer price index, period average & 10.0 & 1.7 & 4.8 & 5.3 & 5.0 & 5.0 & 5.0 & 5.0 \\
\hline Consumer price index, end-of-period & 5.5 & 3.0 & 5.5 & 5.0 & 5.0 & 5.0 & 5.0 & 5.0 \\
\hline GDP per capita (in US\$) & 2,937 & 2,450 & 2,448 & 2,479 & 2,676 & 2,892 & 3,125 & 3,377 \\
\hline \multirow[t]{2}{*}{ Unemployment rate (in percent) } & 16.5 & 16.9 & $\ldots$ & $\ldots$ & $\ldots$ & $\ldots$ & $\ldots$ & $\ldots$ \\
\hline & \multicolumn{8}{|c|}{ (In percent of GDP) } \\
\hline \multicolumn{9}{|l|}{ Investment and saving } \\
\hline Investment 2/ & 21.5 & 14.4 & 15.0 & 17.4 & 18.6 & 19.2 & 20.0 & 20.0 \\
\hline Public & 4.3 & 6.9 & 7.6 & 6.7 & 5.8 & 5.5 & 5.4 & 5.6 \\
\hline Private & 17.2 & 7.6 & 7.4 & 10.7 & 12.8 & 13.7 & 14.6 & 14.4 \\
\hline Gross national saving & -1.2 & 2.6 & 2.4 & 4.2 & 6.0 & 7.6 & 9.3 & 10.4 \\
\hline Public & 2.2 & -0.8 & 2.2 & 2.5 & 2.8 & 3.2 & 3.6 & 3.5 \\
\hline Private & -3.4 & 3.4 & 0.2 & 1.7 & 3.3 & 4.4 & 5.7 & 6.9 \\
\hline Saving-investment balance & -22.7 & -11.9 & -12.6 & -13.2 & -12.6 & -11.6 & -10.7 & -9.6 \\
\hline \multicolumn{9}{|l|}{ Consolidated government operations } \\
\hline Total government debt & 25.0 & 37.1 & 46.1 & 49.3 & 47.1 & 45.1 & 42.2 & 40.5 \\
\hline o/w foreign-currency denominated & 20.9 & 31.5 & 40.6 & 43.2 & 40.5 & 37.0 & 34.1 & 32.7 \\
\hline Revenue $3 /$ & 30.7 & 29.3 & 29.8 & 29.1 & 28.2 & 28.0 & 27.9 & 27.8 \\
\hline Expenses & 28.5 & 30.1 & 27.7 & 26.5 & 25.5 & 24.8 & 24.3 & 24.4 \\
\hline Operating balance & 2.2 & -0.8 & 2.2 & 2.5 & 2.8 & 3.2 & 3.6 & 3.5 \\
\hline Capital spending and net lending & 8.6 & 8.4 & 8.5 & 7.4 & 6.2 & 5.7 & 5.6 & 5.6 \\
\hline Overall balance & -6.3 & -9.2 & -6.3 & -4.8 & -3.4 & -2.5 & -2.0 & -2.1 \\
\hline Statistical discrepancy & 0.0 & 0.0 & 0.0 & 0.0 & 0.0 & 0.0 & 0.0 & 0.0 \\
\hline Total financing & 6.3 & 9.2 & 6.3 & 4.8 & 3.4 & 2.5 & 2.0 & 2.1 \\
\hline Domestic & -2.3 & 3.3 & -0.3 & 1.8 & 0.6 & 1.7 & 0.4 & 0.3 \\
\hline External & 5.0 & 3.9 & 5.6 & 2.4 & 2.5 & 0.6 & 1.3 & 1.8 \\
\hline Privatization receipts & 3.7 & 2.0 & 1.1 & 0.7 & 0.4 & 0.2 & 0.2 & 0.0 \\
\hline & \multicolumn{8}{|c|}{ (Annual percentage change, unless otherwise indicated) } \\
\hline \multicolumn{9}{|l|}{ Monetary sector } \\
\hline Reserve money & -4.5 & 21.8 & 10.0 & $\ldots$ & $\ldots$ & $\ldots$ & $\ldots$ & $\ldots$ \\
\hline Broad money (including fx deposits) & 7.0 & 8.1 & 20.0 & $\cdots$ & $\ldots$ & $\ldots$ & $\cdots$ & $\ldots$ \\
\hline Bank credit to the private sector & 28.2 & -13.5 & 19.7 & $\ldots$ & $\ldots$ & $\ldots$ & $\ldots$ & $\ldots$ \\
\hline Deposit interest rate (annual weighted average on flows) & 9.5 & 9.8 & $\ldots$ & $\ldots$ & $\ldots$ & $\ldots$ & $\ldots$ & $\ldots$ \\
\hline Lending interest rate (annual weighted average on flows) & 21.9 & 22.4 & $\cdots$ & $\cdots$ & $\cdots$ & $\cdots$ & $\cdots$ & $\cdots$ \\
\hline \multicolumn{9}{|l|}{ External sector } \\
\hline Exports of goods and services (percent of GDP) & 28.7 & 29.8 & 35.8 & 39.1 & 39.4 & 39.7 & 39.9 & 40.2 \\
\hline Annual percentage change & 15.9 & -13.3 & 20.2 & 10.8 & 8.7 & 8.9 & 8.9 & 8.9 \\
\hline Imports of goods and services (percent of GDP) & 58.3 & 49.0 & 56.1 & 59.0 & 57.8 & 56.3 & 55.6 & 54.7 \\
\hline Annual percentage change & 26.7 & -29.8 & 14.5 & 6.5 & 5.8 & 5.4 & 6.7 & 6.4 \\
\hline Net imports of oil (in US\$) & 762 & 555 & 668 & 723 & 782 & 839 & 884 & 928 \\
\hline Current account balance (in millions of US\$) & $-2,915$ & $-1,274$ & $-1,355$ & $-1,437$ & $-1,480$ & $-1,469$ & $-1,466$ & $-1,430$ \\
\hline In percent of GDP & -22.7 & -11.9 & -12.6 & -13.2 & -12.6 & -11.6 & -10.7 & -9.6 \\
\hline Gross international reserves (in millions of US\$) & 1,480 & 2,110 & 2,213 & 2,308 & 2,238 & 2,064 & 2,044 & 2,221 \\
\hline In months of next year's imports of goods and services & 3.4 & 4.2 & 4.1 & 4.1 & 3.7 & 3.2 & 3.0 & 3.1 \\
\hline Foreign direct investment (percent of GDP) & 12.2 & 7.1 & 6.5 & 8.2 & 8.6 & 9.0 & 9.4 & 9.4 \\
\hline Average exchange rate (lari per US\$) & 1.48 & 1.67 & $\ldots$ & $\ldots$ & $\ldots$ & $\ldots$ & $\ldots$ & $\ldots$ \\
\hline
\end{tabular}

Sources: Georgian authorities; and Fund staff estimates.

1/ Excludes Abkhazia residents.

$2 /$ Investment is measured on a net basis (acquisitions minus disposals of nonfinancial assets).

$3 /$ Includes grants. 
Table 2. Georgia: Annual General Government Operations, 2009-15 1/

\begin{tabular}{|c|c|c|c|c|c|c|c|c|c|c|c|c|c|c|c|c|}
\hline & \multirow[t]{2}{*}{2009} & \multicolumn{2}{|c|}{2010} & \multirow[t]{2}{*}{2011} & \multirow[t]{2}{*}{2012} & \multirow[t]{2}{*}{2013} & \multirow[t]{2}{*}{2014} & \multirow[t]{2}{*}{2015} & \multirow[t]{2}{*}{2009} & \multicolumn{2}{|c|}{2010} & \multirow[t]{2}{*}{2011} & \multirow[t]{2}{*}{2012} & \multirow[t]{2}{*}{2013} & \multirow[t]{2}{*}{2014} & \multirow[t]{2}{*}{2015} \\
\hline & & $5 \mathrm{Rev}$. & Proj. & & & & & & & 5 Rev. & Proj. & & & & & \\
\hline & \multicolumn{8}{|c|}{ (In millions of lari) } & \multicolumn{8}{|c|}{ (In percent of GDP) } \\
\hline Revenues & 5,264 & 5,518 & 5,865 & 6,238 & 6,685 & 7,310 & 8,027 & 8,823 & 29.3 & 29.1 & 29.8 & 29.1 & 28.2 & 28.0 & 27.9 & 27.8 \\
\hline Taxes & 4,389 & 4,598 & 4,859 & 5,500 & 6,064 & 6,686 & 7,371 & 8,127 & 24.5 & 24.3 & 24.7 & 25.6 & 25.6 & 25.6 & 25.6 & 25.6 \\
\hline Other revenues & 487 & 397 & 440 & 440 & 450 & 470 & 520 & 560 & 2.7 & 2.1 & 2.2 & 2.0 & 1.9 & 1.8 & 1.8 & 1.8 \\
\hline Grants & 389 & 524 & 566 & 297 & 171 & 154 & 136 & 136 & 2.2 & 2.8 & 2.9 & 1.4 & 0.7 & 0.6 & 0.5 & 0.4 \\
\hline Current expenditures & 5,407 & 5,404 & 5,441 & 5,695 & 6,031 & 6,475 & 6,990 & 7,724 & 30.1 & 28.5 & 27.7 & 26.5 & 25.5 & 24.8 & 24.3 & 24.4 \\
\hline Compensation for employees & 1,048 & 1,151 & 1,151 & 1,209 & 1,269 & 1,333 & 1,438 & 1,586 & 5.8 & 6.1 & 5.9 & 5.6 & 5.4 & 5.1 & 5.0 & 5.0 \\
\hline Use of goods and services & 1,105 & 1,062 & 1,062 & 1,100 & 1,150 & 1,230 & 1,340 & 1,477 & 6.2 & 5.6 & 5.4 & 5.1 & 4.9 & 4.7 & 4.7 & 4.7 \\
\hline Subsidies & 420 & 384 & 384 & 380 & 370 & 360 & 360 & 397 & 2.3 & 2.0 & 2.0 & 1.8 & 1.6 & 1.4 & 1.3 & 1.3 \\
\hline Grants & 14 & 12 & 12 & 19 & 20 & 20 & 22 & 24 & 0.1 & 0.1 & 0.1 & 0.1 & 0.1 & 0.1 & 0.1 & 0.1 \\
\hline Social expenses & 1,506 & 1,611 & 1,611 & 1,717 & 1,893 & 2,061 & 2,244 & 2,474 & 8.4 & 8.5 & 8.2 & 8.0 & 8.0 & 7.9 & 7.8 & 7.8 \\
\hline Other expenses & 1,142 & 941 & 969 & 967 & 980 & 1,030 & 1,130 & 1,246 & 6.4 & 5.0 & 4.9 & 4.5 & 4.1 & 3.9 & 3.9 & 3.9 \\
\hline Of which: arrears clearance and provisions & 198 & 29 & 29 & 0 & 0 & 0 & 0 & 0 & 1.1 & 0.2 & 0.1 & 0.0 & 0.0 & 0.0 & 0.0 & 0.0 \\
\hline Interest & 171 & 243 & 252 & 303 & 348 & 441 & 456 & 520 & 1.0 & 1.3 & 1.3 & 1.4 & 1.5 & 1.7 & 1.6 & 1.6 \\
\hline To nonresidents & 113 & 162 & 171 & 184 & 205 & 257 & 234 & 280 & 0.6 & 0.9 & 0.9 & 0.9 & 0.9 & 1.0 & 0.8 & 0.9 \\
\hline To residents & 58 & 81 & 81 & 119 & 144 & 184 & 221 & 240 & 0.3 & 0.4 & 0.4 & 0.6 & 0.6 & 0.7 & 0.8 & 0.8 \\
\hline Operating balance & -143 & 114 & 424 & 542 & 654 & 835 & 1,038 & 1,099 & -0.8 & 0.6 & 2.2 & 2.5 & 2.8 & 3.2 & 3.6 & 3.5 \\
\hline Capital spending and net lending & 1,506 & 1,507 & 1,664 & 1,580 & 1,470 & 1,490 & 1,600 & 1,764 & 8.4 & 8.0 & 8.5 & 7.4 & 6.2 & 5.7 & 5.6 & 5.6 \\
\hline Capital & 1,444 & 1,532 & 1,702 & 1,580 & 1,470 & 1,490 & 1,600 & 1,764 & 8.0 & 8.1 & 8.7 & 7.4 & 6.2 & 5.7 & 5.6 & 5.6 \\
\hline Net lending & 62 & -25 & -37 & 0 & 0 & 0 & 0 & 0 & 0.3 & -0.1 & -0.2 & 0.0 & 0.0 & 0.0 & 0.0 & 0.0 \\
\hline Overall balance & $-1,648$ & $-1,393$ & $-1,241$ & $-1,038$ & -816 & -655 & -562 & -665 & -9.2 & -7.4 & -6.3 & -4.8 & -3.4 & -2.5 & -2.0 & -2.1 \\
\hline Statistical discrepancy & 0 & 0 & 0 & 0 & 0 & 0 & 0 & 0 & 0.0 & 0.0 & 0.0 & 0.0 & 0.0 & 0.0 & 0.0 & 0.0 \\
\hline Total financing & 1,648 & 1,393 & 1,241 & 1,038 & 816 & 655 & 562 & 665 & 9.2 & 7.4 & 6.3 & 4.8 & 3.4 & 2.5 & 2.0 & 2.1 \\
\hline Domestic & 596 & 65 & -62 & 378 & 135 & 437 & 128 & 97 & 3.3 & 0.3 & -0.3 & 1.8 & 0.6 & 1.7 & 0.4 & 0.3 \\
\hline Net T-bill issuance & 260 & 100 & 100 & 293 & 182 & 488 & 184 & 159 & 1.4 & 0.5 & 0.5 & 1.4 & 0.8 & 1.9 & 0.6 & 0.5 \\
\hline Amortization 2/ & -37 & -35 & -35 & -42 & -46 & -51 & -56 & -62 & -0.2 & -0.2 & -0.2 & -0.2 & -0.2 & -0.2 & -0.2 & -0.2 \\
\hline Use of deposits at the NBG and banks & 373 & 0 & -127 & 127 & 0 & 0 & 0 & 0 & 2.1 & 0.0 & -0.6 & 0.6 & 0.0 & 0.0 & 0.0 & 0.0 \\
\hline External & 694 & 1,097 & 1,093 & 510 & 580 & 168 & 385 & 568 & 3.9 & 5.8 & 5.6 & 2.4 & 2.5 & 0.6 & 1.3 & 1.8 \\
\hline Borrowing & 787 & 1,236 & 1,241 & 644 & 694 & 1,729 & 703 & 1,255 & 4.4 & 6.5 & 6.3 & 3.0 & 2.9 & 6.6 & 2.4 & 4.0 \\
\hline Of which: IMF & 170 & 674 & 524 & & & & & & 0.9 & 3.6 & 2.7 & 0.0 & 0.0 & 0.0 & 0.0 & 0.0 \\
\hline Amortization & -133 & -139 & -148 & -134 & -114 & $-1,561$ & -319 & -687 & -0.7 & -0.7 & -0.8 & -0.6 & -0.5 & -6.0 & -1.1 & -2.2 \\
\hline Use of Sovereign Wealth Fund resources & 40 & 0 & 0 & 0 & 0 & 0 & 0 & 0 & 0.2 & 0.0 & 0.0 & 0.0 & 0.0 & 0.0 & 0.0 & 0.0 \\
\hline Privatization receipts & 358 & 230 & 210 & 150 & 100 & 50 & 50 & 0 & 2.0 & 1.2 & 1.1 & 0.7 & 0.4 & 0.2 & 0.2 & 0.0 \\
\hline \multicolumn{17}{|l|}{ Memorandum items: } \\
\hline Nominal GDP & 17,949 & 18,934 & 19,657 & 21,465 & 23,665 & 26,091 & 28,765 & 31,714 & & & & & & & & \\
\hline Fiscal deficit excluding grants & 2,037 & 1,916 & 1,807 & 1,335 & 987 & 809 & 698 & 801 & 11.3 & 10.1 & 9.2 & 6.2 & 4.2 & 3.1 & 2.4 & 2.5 \\
\hline Total expenditures (current prices) 3/ & 6,851 & 6,936 & 7,143 & 7,275 & 7,501 & 7,965 & 8,590 & 9,488 & 38.2 & 36.6 & 36.3 & 33.9 & 31.7 & 30.5 & 29.9 & 29.9 \\
\hline Total expenditures (constant 2008 prices) & 6,994 & 6,843 & 6,958 & 6,750 & 6,628 & 6,702 & 6,884 & 7,242 & & & & & & & & \\
\hline
\end{tabular}


Table 3. Georgia: Quarterly General Government Operations, 2009-10 1/

\begin{tabular}{|c|c|c|c|c|c|c|c|c|c|c|}
\hline & \multicolumn{5}{|c|}{2009} & \multicolumn{5}{|c|}{2010} \\
\hline & $\begin{array}{r}\text { Q1 } \\
\text { Act. }\end{array}$ & $\begin{array}{r}\text { Q2 } \\
\text { Act. }\end{array}$ & $\begin{array}{r}\text { Q3 } \\
\text { Act. }\end{array}$ & $\begin{array}{r}\text { Q4 } \\
\text { Act. }\end{array}$ & $\begin{array}{c}\text { Annual } \\
\text { Act. }\end{array}$ & $\begin{array}{r}\text { Q1 } \\
\text { Act. }\end{array}$ & $\begin{array}{c}\text { Q2 } \\
\text { Proj. }\end{array}$ & $\begin{array}{c}\text { Q3 } \\
\text { Proj. }\end{array}$ & $\begin{array}{c}\text { Q4 } \\
\text { Proj. }\end{array}$ & $\begin{array}{c}\text { Annual } \\
\text { Proj. }\end{array}$ \\
\hline & \multicolumn{10}{|c|}{ (In millions of lari) } \\
\hline Revenues & 1,267 & 1,118 & 1,292 & 1,588 & 5,264 & 1,318 & 1,381 & 1,457 & 1,708 & 5,865 \\
\hline Taxes & 1,139 & 996 & 1,094 & 1,159 & 4,389 & 1,130 & 1,164 & 1,254 & 1,312 & 4,859 \\
\hline Direct & 458 & 452 & 475 & 473 & 1,858 & 511 & 519 & 508 & 509 & 2,047 \\
\hline Indirect & 681 & 544 & 619 & 687 & 2,531 & 619 & 645 & 746 & 803 & 2,812 \\
\hline Other revenues & 79 & 84 & 107 & 217 & 487 & 97 & 130 & 77 & 135 & 440 \\
\hline Grants & 48 & 38 & 90 & 212 & 389 & 92 & 87 & 126 & 261 & 566 \\
\hline Current expenditures & 1,296 & 1,300 & 1,282 & 1,529 & 5,407 & 1,208 & 1,367 & 1,372 & 1,494 & 5,441 \\
\hline Compensation for employees & 248 & 260 & 251 & 289 & 1,048 & 263 & 290 & 280 & 319 & 1,151 \\
\hline Use of goods and services & 207 & 273 & 260 & 365 & 1,105 & 203 & 270 & 280 & 309 & 1,062 \\
\hline Subsidies & 87 & 105 & 104 & 125 & 420 & 85 & 95 & 102 & 102 & 384 \\
\hline Grants & 7 & 3 & 2 & 3 & 14 & 5 & 3 & 2 & 2 & 12 \\
\hline Social expenses & 352 & 377 & 377 & 400 & 1,506 & 399 & 400 & 400 & 411 & 1,611 \\
\hline Other expenses & 370 & 226 & 259 & 288 & 1,142 & 220 & 225 & 260 & 264 & 969 \\
\hline of which arrears clearance and provisions & 171 & 5 & 19 & 3 & 198 & 5 & 4 & 5 & 16 & 29 \\
\hline Interest & 26 & 56 & 30 & 59 & 171 & 33 & 85 & 48 & 87 & 252 \\
\hline To nonresidents & 13 & 40 & 16 & 45 & 113 & 17 & 66 & 28 & 60 & 171 \\
\hline To residents & 13 & 16 & 14 & 15 & 58 & 16 & 19 & 20 & 27 & 81 \\
\hline Operating balance & -30 & -182 & 9 & 59 & -143 & 110 & 14 & 85 & 214 & 424 \\
\hline Capital spending and net lending & 199 & 338 & 474 & 495 & 1,506 & 206 & 386 & 484 & 589 & 1,664 \\
\hline Capital & 169 & 333 & 452 & 490 & 1444 & 233 & 390 & 482 & 597 & 1,702 \\
\hline Net lending & 30 & 5 & 22 & 5 & 62 & -27 & -4 & 2 & -8 & -37 \\
\hline Overall balance & -228 & -520 & -464 & -436 & $-1,648$ & -96 & -371 & -399 & -374 & $-1,241$ \\
\hline Statistical discrepancy & 0 & 0 & 0 & 0 & 0 & 0 & 0 & 0 & 0 & 0 \\
\hline Total financing & 228 & 520 & 464 & 436 & 1,648 & 96 & 371 & 399 & 374 & 1,241 \\
\hline Domestic & 63 & 375 & 103 & 55 & 596 & -348 & 275 & -90 & 100 & -62 \\
\hline Net T-Bill issuance & 0 & 0 & 68 & 192 & 260 & 67 & 74 & -11 & -30 & 100 \\
\hline Amortization 2/ & -9 & -11 & -12 & -5 & -37 & -12 & -10 & -6 & -7 & -35 \\
\hline Use of deposits at the NBG and banks & 72 & 386 & 48 & -132 & 373 & -403 & 211 & -73 & 137 & -127 \\
\hline External & 64 & 40 & 301 & 289 & 694 & 411 & 66 & 435 & 181 & 1,093 \\
\hline Borrowing & 37 & 60 & 377 & 313 & 787 & 424 & 93 & 514 & 210 & 1,241 \\
\hline of which IMF & 0 & 0 & 0 & 170 & 170 & 385 & 0 & 139 & 0 & 524 \\
\hline Amortization & -13 & -20 & -76 & -24 & -133 & -13 & -26 & -79 & -29 & -148 \\
\hline Use of Sovereign Wealth Fund resources & 40 & 0 & 0 & 0 & 40 & 0 & 0 & 0 & 0 & 0 \\
\hline Privatization receipts & 101 & 106 & 60 & 91 & 358 & 33 & 30 & 54 & 93 & 210 \\
\hline Memorandum items: & & & & & & & & & & \\
\hline Nominal GDP & 3,889 & 4,247 & 4,578 & 5,234 & 17,949 & 4,205 & 4,816 & 5,174 & 5,463 & 19,657 \\
\hline
\end{tabular}

Sources: Ministry of Finance; and Fund staff estimates.

1/ General government includes central and local governments and the Sovereign Wealth Funds.

2/ Excluding arrears clearance, provisions and T-bill repayment. 
Table 4. Georgia: Summary Balance of Payments, 2008-15

(In millions of U.S. dollars)

\begin{tabular}{|c|c|c|c|c|c|c|c|c|c|c|}
\hline & \multirow[t]{2}{*}{2008} & \multicolumn{2}{|l|}{2009} & \multicolumn{2}{|c|}{2010} & \multirow{2}{*}{$\begin{array}{l}2011 \\
\text { Proj. }\end{array}$} & \multirow{2}{*}{$\begin{array}{l}2012 \\
\text { Proj. }\end{array}$} & \multirow{2}{*}{$\begin{array}{l}2013 \\
\text { Proj. }\end{array}$} & \multirow{2}{*}{$\begin{array}{l}2014 \\
\text { Proj. }\end{array}$} & \multirow{2}{*}{$\begin{array}{l}2015 \\
\text { Proj. }\end{array}$} \\
\hline & & 5th Review & & 5th Review & Proj. & & & & & \\
\hline Current account balance & $-2,915$ & $-1,312$ & $-1,274$ & $-1,544$ & $-1,355$ & $-1,437$ & $-1,480$ & $-1,469$ & $-1,466$ & $-1,430$ \\
\hline Trade balance & $-3,833$ & $-2,415$ & $-2,400$ & $-2,664$ & $-2,446$ & $-2,454$ & $-2,516$ & $-2,539$ & $-2,644$ & $-2,761$ \\
\hline Exports & 2,428 & 1,844 & 1,893 & 2,124 & 2,452 & 2,738 & 2,955 & 3,213 & 3,490 & 3,772 \\
\hline Imports & $-6,261$ & $-4,260$ & $-4,293$ & $-4,789$ & $-4,898$ & $-5,191$ & $-5,471$ & $-5,752$ & $-6,134$ & $-6,532$ \\
\hline Services & 23 & 262 & 332 & 274 & 261 & 293 & 352 & 421 & 496 & 614 \\
\hline Services: credit & 1,260 & 1,258 & 1,306 & 1,391 & 1,395 & 1,525 & 1,679 & 1,835 & 2,005 & 2,215 \\
\hline Services: debit & $-1,238$ & -996 & -974 & $-1,117$ & $-1,134$ & $-1,232$ & $-1,327$ & $-1,415$ & $-1,510$ & $-1,601$ \\
\hline Income (net) & -165 & -86 & -158 & -111 & -285 & -308 & -343 & -390 & -402 & -412 \\
\hline Of which: interest payments & -248 & -261 & -246 & -306 & -323 & -352 & -378 & -412 & -412 & -425 \\
\hline Transfers (net) & 1,060 & 928 & 952 & 957 & 1,115 & 1,031 & 1,027 & 1,039 & 1,084 & 1,129 \\
\hline Of which: public sector & 365 & 167 & 134 & 204 & 225 & 119 & 80 & 54 & 53 & 52 \\
\hline Capital account & 105 & 170 & 178 & 209 & 231 & 90 & 78 & 69 & 60 & 59 \\
\hline Financial account & 2,808 & 1,555 & 1,545 & 1,056 & 853 & 1,394 & 1,571 & 1,601 & 1,742 & 1,752 \\
\hline Direct investment (net) & 1,523 & 767 & 765 & 858 & 694 & 888 & 1,007 & 1,144 & 1,274 & 1,377 \\
\hline Monetary authorities, net $1 /$ & 21 & 239 & 247 & 0 & 0 & 0 & 0 & 0 & 0 & 0 \\
\hline General government & 651 & 385 & 384 & 251 & 324 & 259 & 294 & 284 & 262 & 369 \\
\hline Private Sector, excl. FDI & 612 & 163 & 149 & -53 & -165 & 248 & 269 & 174 & 207 & 6 \\
\hline Banks & 403 & 1 & 37 & -76 & 46 & 122 & 106 & 128 & 206 & 64 \\
\hline Portfolio investment, net & 109 & 7 & 7 & 18 & 1 & 5 & 2 & 13 & 12 & 12 \\
\hline Of which: equity liabilities & 101 & 7 & 7 & 18 & 1 & 5 & 7 & 10 & 12 & 13 \\
\hline Loans received (net) & 596 & -206 & -228 & -73 & -46 & 102 & 84 & 90 & 164 & 21 \\
\hline Other, net (currency and deposits) & -302 & 200 & 258 & -20 & 92 & 15 & 20 & 25 & 30 & 32 \\
\hline Other sectors & 209 & 162 & 112 & 23 & -211 & 126 & 163 & 46 & 1 & -58 \\
\hline Portfolio investment, net & 14 & 14 & -2 & 33 & -2 & -3 & 7 & 8 & 19 & 20 \\
\hline Long-term loans received (net) & 59 & 53 & 97 & 26 & -73 & 49 & 63 & 74 & 24 & -88 \\
\hline Other, net & 137 & 96 & 17 & -36 & -136 & 79 & 93 & -36 & -42 & 10 \\
\hline Errors and omissions & -49 & 19 & 13 & 0 & 0 & 0 & 0 & 0 & 0 & 0 \\
\hline Overall balance & -51 & 431 & 462 & -280 & -271 & 48 & 168 & 201 & 337 & 382 \\
\hline Financing & 51 & -431 & -462 & 280 & 271 & -48 & -168 & -201 & -337 & -382 \\
\hline Gross International Reserves (-increase) & -131 & -619 & -616 & -168 & -154 & -95 & 70 & 174 & 20 & -177 \\
\hline Use of Fund Resources & 222 & 313 & 313 & 447 & 425 & 47 & -238 & -375 & -357 & -205 \\
\hline Purchases (SBA) & 257 & 340 & 340 & 470 & 446 & 104 & 0 & 0 & 0 & 0 \\
\hline Of which: augmentation & & & & 319 & 301 & 104 & & $\ldots$ & $\ldots$ & \\
\hline Repayments (SBA and ECF 2/) & -35 & -28 & -28 & -22 & -21 & -57 & -238 & -375 & -357 & -205 \\
\hline Exceptional financing & -39 & -124 & -159 & 0 & 0 & 0 & 0 & 0 & 0 & 0 \\
\hline \multicolumn{11}{|l|}{ Memorandum items: } \\
\hline Nominal GDP & 12,870 & 10,737 & 10,745 & 10,896 & 10,745 & 10,889 & 11,762 & 12,721 & 13,757 & 14,879 \\
\hline Current account balance (percent of GDP) & -22.7 & -12.2 & -11.9 & -14.2 & -12.6 & -13.2 & -12.6 & -11.6 & -10.7 & -9.6 \\
\hline excluding official transfers (percent of GDP) & -25.5 & -13.8 & -13.1 & -16.0 & -14.7 & -14.3 & -13.3 & -12.0 & -11.0 & -10.0 \\
\hline Trade balance (in percent of GDP) & -29.8 & -22.5 & -22.3 & -24.5 & -22.8 & -22.5 & -21.4 & -20.0 & -19.2 & -18.6 \\
\hline GNFS exports growth (percent) & 15.9 & -15.9 & -13.3 & 13.3 & 20.2 & 10.8 & 8.7 & 8.9 & 8.9 & 8.9 \\
\hline GNFS exports volume growth (percent) & -1.0 & -2.6 & 0.4 & 6.3 & 4.4 & 9.1 & 8.6 & 8.1 & 8.3 & 8.2 \\
\hline GNFS imports growth (percent) & 26.7 & -29.9 & -29.8 & 12.4 & 14.5 & 6.5 & 5.8 & 5.4 & 6.7 & 6.4 \\
\hline GNFS imports volume growth (percent) & 8.3 & -17.4 & -17.5 & 4.6 & 5.4 & 4.6 & 5.7 & 5.1 & 5.2 & 5.1 \\
\hline Net capital inflows to private sector & 2,135 & 931 & 913 & 805 & 529 & 1,136 & 1,276 & 1,318 & 1,481 & 1,384 \\
\hline (in percent of GDP) & 16.6 & 8.7 & 8.5 & 7.4 & 4.9 & 10.4 & 10.8 & 10.4 & 10.8 & 9.3 \\
\hline Gross international reserves (end of period) & 1,480 & 2,110 & 2,110 & 2,278 & 2,213 & 2,308 & 2,238 & 2,064 & 2,044 & 2,221 \\
\hline (in months of next year GNFS imports) & 3.4 & 4.3 & 4.2 & 4.3 & 4.1 & 4.1 & 3.7 & 3.2 & 3.0 & 3.1 \\
\hline External debt (nominal) & 5,712 & 5,945 & 6,274 & 6,830 & 7,391 & 7,944 & 8,115 & 8,207 & 8,426 & 8,580 \\
\hline (in percent of GDP) & 44.4 & 55.4 & 58.4 & 62.7 & 68.8 & 73.0 & 69.0 & 64.5 & 61.2 & 57.7 \\
\hline MLT External debt service & 590 & 758 & 768 & 766 & 719 & 749 & 1,020 & 2,024 & 1,690 & 1,658 \\
\hline (in percent of exports) & 16.0 & 24.4 & 24.0 & 21.8 & 18.7 & 17.6 & 22.0 & 40.1 & 30.7 & 27.7 \\
\hline External public sector debt (nominal) & 2,691 & 3,413 & 3,382 & 4,253 & 4,366 & 4,702 & 4,759 & 4,711 & 4,696 & 4,861 \\
\hline (in percent of GDP) & 20.9 & 31.8 & 31.5 & 39.0 & 40.6 & 43.2 & 40.5 & 37.0 & 34.1 & 32.7 \\
\hline External public debt service & 125 & 192 & 168 & 242 & 197 & 235 & 426 & 1,095 & 581 & 601 \\
\hline (in percent of exports) & 3.4 & 6.2 & 5.2 & 6.0 & 5.1 & 5.5 & 9.2 & 21.7 & 10.6 & 10.0 \\
\hline
\end{tabular}

Sources: National Bank of Georgia, Ministry of Finance, and Fund staff estimates.

1/ SDR allocation included under monetary authorities' long-term liabilities.

2/ Following the Low Income Countries (LIC) reforms, effective January 7 2010, the PRGF arrangements were renamed Extended Credit Facility (ECF) arrangements. 
Table 5. Georgia: Accounts of the National Bank of Georgia, 2008-10

\begin{tabular}{|c|c|c|c|c|c|c|c|c|c|c|c|c|}
\hline & \multicolumn{4}{|c|}{2008} & \multicolumn{4}{|c|}{2009} & \multicolumn{4}{|c|}{2010} \\
\hline & Mar. & Jun. & Sep. & Dec. & $\begin{array}{l}\text { Mar. } \\
\text { Act. }\end{array}$ & $\begin{array}{l}\text { Jun. } \\
\text { Act. }\end{array}$ & $\begin{array}{l}\text { Sep. } \\
\text { Act. }\end{array}$ & $\begin{array}{l}\text { Dec. } \\
\text { Act. }\end{array}$ & $\begin{array}{l}\text { Mar. } \\
\text { Act. }\end{array}$ & $\begin{array}{l}\text { Jun. } \\
\text { Proj. } \\
\end{array}$ & $\begin{array}{l}\text { Sep. } \\
\text { Proj. }\end{array}$ & $\begin{array}{l}\text { Dec. } \\
\text { Proj. }\end{array}$ \\
\hline & \multicolumn{12}{|c|}{ (In millions of lari) } \\
\hline Net foreign exchange position & 1,500 & 1,594 & 1,266 & 1,528 & 1,313 & 1,303 & 1,489 & 1,842 & 2,117 & 1,734 & 2,113 & 2,132 \\
\hline Gross International Reserves & 2,099 & 2,164 & 1,930 & 2,467 & 2,493 & 2,517 & 3,371 & 3,558 & 3,847 & 3,508 & 3,960 & 4,316 \\
\hline Other foreign assets & 76 & 70 & 87 & 41 & 35 & 14 & 15 & 5 & 6 & 6 & 6 & 7 \\
\hline Foreign liabilities & -675 & -640 & -750 & -981 & $-1,215$ & $-1,228$ & $-1,896$ & $-1,722$ & $-1,736$ & $-1,780$ & $-1,854$ & $-2,190$ \\
\hline Of which: use of Fund resources & -361 & -342 & -655 & -769 & $-1,040$ & $-1,068$ & $-1,330$ & $-1,149$ & $-1,143$ & $-1,193$ & $-1,201$ & $-1,517$ \\
\hline Of which: compulsory reserves in USD & -282 & -296 & -88 & -121 & -125 & -109 & -122 & -127 & -142 & -149 & -241 & -250 \\
\hline Of which: swap liabilities & 0 & 0 & 0 & 0 & 0 & -30 & -61 & -61 & -63 & -33 & 0 & 0 \\
\hline Net domestic assets & -87 & 6 & 468 & -96 & -47 & 226 & 201 & -98 & -484 & -114 & -371 & -214 \\
\hline Net claims on general government & 315 & 149 & 400 & -103 & -34 & 398 & 441 & 173 & -194 & -64 & -218 & 19 \\
\hline Claims on general government (incl. T-bills) & 776 & 776 & 778 & 777 & 777 & 778 & 764 & 758 & 746 & 736 & 730 & 723 \\
\hline Nontradable govt. debt & 689 & 689 & 689 & 689 & 641 & 641 & 641 & 641 & 601 & 601 & 601 & 601 \\
\hline Securitized debt (marketable) & 87 & 87 & 89 & 88 & 136 & 137 & 123 & 118 & 146 & 136 & 130 & 123 \\
\hline Deposits & -461 & -627 & -377 & -880 & -812 & -380 & -323 & -585 & -940 & -800 & -949 & -704 \\
\hline Claims on rest of economy & 3 & 3 & 3 & 4 & 4 & 4 & 4 & 3 & 3 & 3 & 3 & 3 \\
\hline Claims on banks & -392 & -180 & -18 & 132 & 90 & -17 & -41 & -114 & -89 & 124 & 40 & -12 \\
\hline Bank refinancing & 75 & 0 & 125 & 209 & 204 & 102 & 45 & 45 & 45 & 165 & 215 & 215 \\
\hline Certificates of deposits and bonds & -467 & -180 & -143 & -77 & -114 & -148 & -146 & -219 & -194 & -71 & -175 & -227 \\
\hline Other items, net & -13 & 34 & 82 & -129 & -106 & -160 & -203 & -160 & -204 & -178 & -195 & -225 \\
\hline Reserve money & 1,414 & 1,600 & 1,734 & 1,432 & 1,267 & 1,529 & 1,691 & 1,744 & 1,633 & 1,620 & 1,741 & 1,918 \\
\hline Currency in circulation & 1,287 & 1,413 & 1,399 & 1,291 & 1,141 & 1,201 & 1,286 & 1,458 & 1,399 & 1,420 & 1,520 & 1,670 \\
\hline \multirow[t]{2}{*}{ Bank lari reserves $1 /$} & 126 & 187 & 335 & 141 & 126 & 328 & 405 & 286 & 235 & 200 & 221 & 248 \\
\hline & \multicolumn{12}{|c|}{ (Percent contribution, compared to reserve money at the end of previous year) } \\
\hline Net foreign assets & 2.7 & 8.9 & -13.0 & 4.5 & -15.0 & -15.7 & -2.7 & 21.9 & 15.8 & -6.1 & 15.5 & 16.7 \\
\hline Net domestic assets & -8.3 & -2.2 & 28.7 & -8.9 & 3.4 & 22.5 & 20.8 & -0.1 & -22.2 & -0.9 & -15.7 & -6.7 \\
\hline Net claims on general government & -0.9 & -12.0 & 4.8 & -28.8 & 4.8 & 35.0 & 38.0 & 19.3 & -21.1 & -13.6 & -22.5 & -8.8 \\
\hline Claims on rest of economy & -6.6 & -6.6 & -6.6 & -6.6 & 0.0 & 0.0 & 0.0 & 0.0 & 0.0 & 0.0 & 0.0 & 0.0 \\
\hline Claims on banks & -5.9 & 8.2 & 19.1 & 29.1 & -2.9 & -10.4 & -12.1 & -17.2 & 1.4 & 13.7 & 8.8 & 5.9 \\
\hline \multirow[t]{2}{*}{ Other items, net } & 5.1 & 8.2 & 11.5 & -2.6 & 1.6 & -2.2 & -5.1 & -2.2 & -2.5 & -1.0 & -2.0 & -3.7 \\
\hline & \multicolumn{12}{|c|}{ (Percentage change, relative to end of previous year) } \\
\hline Reserve money & -5.7 & 6.8 & 15.7 & -4.5 & -11.5 & 6.8 & 18.1 & 21.8 & -6.3 & -7.1 & -0.1 & 10.0 \\
\hline Currency in circulation & -1.8 & 7.8 & 6.8 & -1.5 & -11.6 & -6.9 & -0.4 & 13.0 & -4.1 & -2.6 & 4.3 & 14.5 \\
\hline Bank lari reserves $1 /$ & -32.4 & 0.0 & 79.3 & -24.5 & -11.0 & 132.0 & 186.9 & 102.5 & -18.0 & -29.9 & -22.6 & -13.2 \\
\hline \multirow{2}{*}{\multicolumn{13}{|c|}{ Memorandum items: }} \\
\hline & & & & & & & & & & & & \\
\hline (in millions of USD, at prog. exchange rates) 2/ & 747 & 1,055 & 848 & 909 & 777 & 775 & 876 & 987 & 897 & 639 & 740 & 721 \\
\hline Net domestic assets (in millions of lari, at prog. exchange rate) $2 /$ & -87 & 6 & 452 & 116 & -66 & 189 & 228 & 95 & 135 & 553 & 506 & 714 \\
\hline Reserve money (in percent, 12-month growth) & 22.6 & 22.4 & 25.7 & -4.5 & -10.4 & -4.5 & -2.5 & 21.8 & 28.9 & 6.0 & 3.0 & 10.0 \\
\hline
\end{tabular}

Sources: National Bank of Georgia; and Fund staff estimates.

$1 /$ Comprises of required and excess reserves on lari-denominated deposits.

2/ Based on program definition as defined in the TMU. 
Table 6. Georgia: Monetary Survey, 2008-10

\begin{tabular}{|c|c|c|c|c|c|c|c|c|c|c|c|c|}
\hline & \multicolumn{4}{|c|}{2008} & \multicolumn{4}{|c|}{2009} & \multicolumn{4}{|c|}{2010} \\
\hline & Mar. & Jun. & Sep. & Dec. & $\begin{array}{l}\text { Mar. } \\
\text { Act. }\end{array}$ & $\begin{array}{l}\text { Jun. } \\
\text { Act. }\end{array}$ & $\begin{array}{l}\text { Sep. } \\
\text { Act. }\end{array}$ & $\begin{array}{l}\text { Dec. } \\
\text { Act. }\end{array}$ & $\begin{array}{l}\text { Mar. } \\
\text { Act. }\end{array}$ & $\begin{array}{l}\text { Jun. } \\
\text { Proj. }\end{array}$ & $\begin{array}{l}\text { Sep. } \\
\text { Proj. }\end{array}$ & $\begin{array}{l}\text { Dec. } \\
\text { Proj. }\end{array}$ \\
\hline & \multicolumn{12}{|c|}{ (In millions of lari) } \\
\hline Net foreign assets & 481 & 256 & -306 & 79 & -356 & -357 & 240 & 789 & 1,067 & 614 & 939 & 874 \\
\hline NBG & 1,783 & 1,890 & 1,355 & 1,649 & 1,438 & 1,442 & 1,672 & 2,030 & 2,323 & 1,917 & 2,354 & 2,382 \\
\hline Commercial banks & $-1,301$ & $-1,634$ & $-1,660$ & $-1,570$ & $-1,794$ & $-1,800$ & $-1,432$ & $-1,241$ & $-1,256$ & $-1,304$ & $-1,415$ & $-1,508$ \\
\hline Of which: liabilities & $-2,096$ & $-2,538$ & $-2,504$ & $-2,901$ & $-2,760$ & $-2,446$ & $-2,319$ & $-2,229$ & $-2,197$ & $-2,299$ & $-2,354$ & $-2,473$ \\
\hline Net domestic assets & 3,545 & 3,932 & 4,272 & 4,179 & 4,031 & 3,952 & 3,852 & 3,814 & 3,669 & 4,346 & 4,340 & 4,649 \\
\hline Domestic credit & 5,336 & 5,682 & 5,846 & 5,921 & 5,644 & 5,733 & 5,676 & 5,539 & 5,418 & 6,131 & 6,172 & 6,538 \\
\hline Net claims on general government & 265 & 85 & 339 & -155 & -114 & 270 & 387 & 280 & -5 & 199 & 33 & 241 \\
\hline Of which: government deposits at NBG & -461 & -627 & -377 & -880 & -812 & -380 & -323 & -585 & -940 & -800 & -949 & -704 \\
\hline Of which: T-bills at commercial banks & & & & & & & 68 & 260 & 326 & 400 & 389 & 359 \\
\hline Credit to the rest of the economy & 5,071 & 5,598 & 5,507 & 6,076 & 5,758 & 5,462 & 5,289 & 5,259 & 5,423 & 5,932 & 6,138 & 6,296 \\
\hline Other items, net & $-1,791$ & $-1,750$ & $-1,574$ & $-1,742$ & $-1,613$ & $-1,781$ & $-1,824$ & $-1,725$ & $-1,749$ & $-1,785$ & $-1,832$ & $-1,888$ \\
\hline Broad money (M3) & 4,027 & 4,188 & 3,966 & 4,258 & 3,675 & 3,594 & 4,092 & 4,603 & 4,736 & 4,960 & 5,279 & 5,523 \\
\hline Broad money, excl. forex deposits (M2) & 2,259 & 2,417 & 2,257 & 1,854 & 1,634 & 1,709 & 1,914 & 2,133 & 2,215 & 2,305 & 2,470 & 2,648 \\
\hline Currency held by the public & 1,123 & 1,235 & 1,196 & 1,083 & 960 & 1,020 & 1,093 & 1,229 & 1,187 & 1,223 & 1,323 & 1,473 \\
\hline \multirow[t]{2}{*}{ Total deposit liabilities } & 2,904 & 2,952 & 2,769 & 3,176 & 2,715 & 2,574 & 2,999 & 3,373 & 3,549 & 3,737 & 3,955 & 4,050 \\
\hline & \multicolumn{12}{|c|}{ (Percent contribution, compared to broad money at the end of previous year) } \\
\hline Net foreign assets & 5.2 & -0.5 & -14.6 & -4.9 & -10.2 & -10.3 & 3.8 & 16.7 & 6.0 & -3.8 & 3.3 & 1.8 \\
\hline Net domestic assets & -4.0 & 5.7 & 14.2 & 11.9 & -3.5 & -5.3 & -7.7 & -8.6 & -3.1 & 11.6 & 11.4 & 18.2 \\
\hline Domestic credit & 7.6 & 16.3 & 20.4 & 22.3 & -6.5 & -4.4 & -5.8 & -9.0 & -2.6 & 12.9 & 13.7 & 21.7 \\
\hline Net claims on general government & -0.7 & -5.2 & 1.1 & -11.3 & 1.0 & 10.0 & 12.7 & 10.2 & -6.2 & -1.8 & -5.4 & -0.8 \\
\hline Credit to the rest of the economy & 8.3 & 21.6 & 19.3 & 33.6 & -7.5 & -14.4 & -18.5 & -19.2 & 3.6 & 14.6 & 19.1 & 22.5 \\
\hline \multirow[t]{2}{*}{ Other items, net } & -11.7 & -10.7 & -6.2 & -10.5 & 3.0 & -0.9 & -1.9 & 0.4 & -0.5 & -1.3 & -2.3 & -3.5 \\
\hline & \multicolumn{12}{|c|}{ (Percentage change, relative to end of previous year) } \\
\hline Broad money (M3) & 1.2 & 5.2 & -0.4 & 7.0 & -13.7 & -15.6 & -3.9 & 8.1 & 2.9 & 7.8 & 14.7 & 20.0 \\
\hline Broad money, excl. forex deposits (M2) & 6.0 & 13.4 & 5.9 & -13.0 & -11.9 & -7.8 & 3.2 & 15.0 & 3.9 & 8.1 & 15.8 & 24.2 \\
\hline Currency held by the public & -2.5 & 7.2 & 3.9 & -6.0 & -11.3 & -5.7 & 1.0 & 13.6 & -3.5 & -0.5 & 7.6 & 19.8 \\
\hline Total deposit liabilities & 2.7 & 4.4 & -2.1 & 12.3 & -14.5 & -19.0 & -5.6 & 6.2 & 5.2 & 10.8 & 17.3 & 20.1 \\
\hline Credit to the rest of the economy & 7.0 & 18.1 & 16.2 & 28.2 & -5.2 & -10.1 & -13.0 & -13.5 & 3.1 & 12.8 & 16.7 & 19.7 \\
\hline \multicolumn{13}{|l|}{ Memorandum items: } \\
\hline M3 (in percent, 12-month growth) & 50.6 & 28.9 & 6.9 & 7.0 & -8.7 & -14.2 & 3.2 & 8.1 & 28.9 & 38.0 & 29.0 & 20.0 \\
\hline M2 (in percent, 12-month growth) & 65.4 & 46.8 & 17.1 & -13.0 & -27.7 & -29.3 & -15.2 & 15.0 & 35.6 & 34.9 & 29.1 & 24.2 \\
\hline Credit to the economy (in percent, 12-month growth) & 62.4 & 53.9 & 28.3 & 28.2 & 13.6 & -2.4 & -4.0 & -13.5 & -5.8 & 8.6 & 16.1 & 19.7 \\
\hline Ratio of bank lari reserves to lari deposits $1 /$ & 11.1 & 15.8 & 31.6 & 18.3 & 18.6 & 47.6 & 49.4 & 31.7 & 22.8 & 18.5 & 19.3 & 21.1 \\
\hline M3 multiplier & 2.20 & 2.07 & 2.13 & 2.61 & 2.49 & 2.10 & 2.16 & 2.35 & 2.52 & 2.61 & 2.59 & 2.47 \\
\hline M3 velocity & 4.41 & 4.45 & 4.76 & 4.48 & 5.11 & 5.03 & 4.37 & 3.90 & 3.86 & 3.80 & 3.68 & 3.56 \\
\hline Foreign exchange deposits in percent of total deposits & 60.9 & 60.0 & 61.7 & 75.7 & 75.2 & 73.2 & 72.6 & 73.2 & 71.0 & 71.0 & 71.0 & 71.0 \\
\hline
\end{tabular}

Sources: National Bank of Georgia; and Fund staff estimates.

$1 /$ Comprises of required and excess reserves on lari-denominated deposits. 
Table 7. Georgia: External Vulnerability Indicators, 2008-15

\begin{tabular}{|c|c|c|c|c|c|c|c|c|}
\hline & $\begin{array}{r}2008 \\
\text { Act. }\end{array}$ & $\begin{array}{r}2009 \\
\text { Act. }\end{array}$ & $\begin{array}{l}2010 \\
\text { Proj. }\end{array}$ & $\begin{array}{l}2011 \\
\text { Proj. }\end{array}$ & $\begin{array}{l}2012 \\
\text { Proj. }\end{array}$ & $\begin{array}{l}2013 \\
\text { Proj. }\end{array}$ & $\begin{array}{l}2014 \\
\text { Proj. }\end{array}$ & $\begin{array}{l}2015 \\
\text { Proj. }\end{array}$ \\
\hline Value of exports of goods and services, percent change & 15.9 & -13.3 & 20.2 & 10.8 & 8.7 & 8.9 & 8.9 & 8.9 \\
\hline Value of imports of goods and services, percent change & 26.7 & -29.8 & 14.5 & 6.5 & 5.8 & 5.4 & 6.7 & 6.4 \\
\hline Terms of trade (deterioration - ) & 0.1 & 1.4 & 5.9 & -0.2 & 0.0 & 0.5 & -0.8 & -0.5 \\
\hline Current account balance (percent of GDP) & -22.7 & -11.9 & -12.6 & -13.2 & -12.6 & -11.6 & -10.7 & -9.6 \\
\hline Capital and financial account (percent of GDP) & 22.6 & 16.0 & 10.1 & 13.6 & 14.0 & 13.1 & 13.1 & 12.2 \\
\hline External public debt (percent of GDP) & 20.9 & 31.5 & 40.6 & 43.2 & 40.5 & 37.0 & 34.1 & 32.7 \\
\hline in percent of exports of goods and services & 73.0 & 105.7 & 113.5 & 110.3 & 102.7 & 93.3 & 85.5 & 81.2 \\
\hline \multicolumn{9}{|l|}{ Debt service on external public debt } \\
\hline (in percent of exports of goods and services) & 3.4 & 5.2 & 5.1 & 5.5 & 9.2 & 21.7 & 10.6 & 10.0 \\
\hline External debt (percent of GDP) & 44.4 & 58.4 & 68.8 & 73.0 & 69.0 & 64.5 & 61.2 & 57.7 \\
\hline in percent of exports of goods and services & 154.9 & 196.1 & 192.1 & 186.4 & 175.1 & 162.6 & 153.3 & 143.3 \\
\hline \multicolumn{9}{|l|}{ Debt service on MLT external debt } \\
\hline (in percent of exports of goods and services) & 16.0 & 24.0 & 18.7 & 17.6 & 22.0 & 40.1 & 30.7 & 27.7 \\
\hline \multicolumn{9}{|l|}{ Gross international reserves } \\
\hline in millions of USD & 1,480 & 2,110 & 2,213 & 2,308 & 2,238 & 2,064 & 2,044 & 2,221 \\
\hline in months of next year's imports of goods and servces & 3.4 & 4.2 & 4.1 & 4.1 & 3.7 & 3.2 & 3.0 & 3.1 \\
\hline in percent of external debt & 25.9 & 33.6 & 29.9 & 29.1 & 27.6 & 25.2 & 24.3 & 25.9 \\
\hline in percent of short-term external debt (remaining maturity) & 100 & 163 & 168 & 138 & 87 & 96 & 95 & 106 \\
\hline
\end{tabular}

Source: Fund staff estimates and projections. 
Table 8. Georgia: Indicators of Fund Credit, 2008-17

(In millions of SDR)

\begin{tabular}{|c|c|c|c|c|c|c|c|c|c|c|}
\hline & 2008 & 2009 & $\begin{array}{l}2010 \\
\text { Proj. }\end{array}$ & $\begin{array}{l}2011 \\
\text { Proj. }\end{array}$ & $\begin{array}{l}2012 \\
\text { Proj. }\end{array}$ & $\begin{array}{l}2013 \\
\text { Proj. }\end{array}$ & $\begin{array}{l}2014 \\
\text { Proj. }\end{array}$ & $\begin{array}{l}2015 \\
\text { Proj. }\end{array}$ & $\begin{array}{l}2016 \\
\text { Proj. }\end{array}$ & $\begin{array}{l}2017 \\
\text { Proj. }\end{array}$ \\
\hline \multicolumn{11}{|l|}{ Existing Fund credit } \\
\hline Stock $1 /$ & 298.8 & 501.6 & 632.1 & 594.0 & 434.1 & 189.2 & 47.5 & 14.0 & 4.2 & 0.0 \\
\hline ECF 2/ & 137.1 & 119.1 & 105.0 & 87.1 & 67.2 & 47.6 & 29.4 & 14.0 & 4.2 & 0.0 \\
\hline SBA & 161.7 & 382.5 & 527.1 & 506.9 & 366.9 & 141.6 & 18.1 & 0.0 & 0.0 & 0.0 \\
\hline Obligations & 23.3 & 22.5 & 17.4 & 44.7 & 166.1 & 248.9 & 143.1 & 33.6 & 9.8 & 4.2 \\
\hline ECF 2/ & 22.8 & 18.6 & 14.1 & 17.9 & 20.1 & 19.7 & 18.3 & 15.5 & 9.8 & 4.2 \\
\hline SBA 3/ & 0.5 & 3.9 & 3.3 & 26.8 & 146.0 & 229.2 & 124.8 & 18.2 & 0.0 & 0.0 \\
\hline \multicolumn{11}{|l|}{ Prospective purchases under the SBA } \\
\hline Disbursements & $\ldots$ & $\ldots$ & 150.0 & 70.0 & 0.0 & 0.0 & 0.0 & 0.0 & 0.0 & 0.0 \\
\hline Stock $1 /$ & $\ldots$ & $\ldots$ & 150.0 & 220.0 & 220.0 & 213.8 & 116.9 & 13.1 & 0.0 & 0.0 \\
\hline Obligations $3 /$ & $\ldots$ & $\ldots$ & 0.2 & 2.3 & 2.7 & 9.0 & 99.1 & 104.7 & 13.2 & 0.0 \\
\hline Principal (repurchases) & $\ldots$ & $\ldots$ & 0.0 & 0.0 & 0.0 & 6.3 & 96.9 & 103.8 & 13.1 & 0.0 \\
\hline GRA charges & $\ldots$ & $\ldots$ & 0.2 & 2.3 & 2.7 & 2.7 & 2.3 & 1.0 & 0.1 & 0.0 \\
\hline Surcharges & $\ldots$ & $\ldots$ & 1.1 & 5.3 & 5.0 & 2.0 & 0.0 & 0.0 & 0.0 & 0.0 \\
\hline Stock of existing and prospective Fund credit $1 /$ & 298.8 & 501.6 & 782.1 & 814.0 & 654.1 & 403.0 & 164.4 & 27.1 & 4.2 & 0.0 \\
\hline In percent of quota & 198.8 & 333.7 & 520.4 & 541.6 & 435.2 & 268.1 & 109.3 & 18.0 & 2.8 & 0.0 \\
\hline In percent of GDP & 3.7 & 7.2 & 11.0 & 11.1 & 8.3 & 4.7 & 1.8 & 0.3 & 0.0 & 0.0 \\
\hline In percent of exports of goods and nonfactor services & 12.8 & 24.2 & 30.6 & 28.4 & 21.0 & 11.9 & 4.5 & 0.7 & 0.1 & 0.0 \\
\hline In percent of gross reserves & 31.9 & 36.7 & 53.2 & 52.5 & 43.6 & 29.1 & 12.0 & 1.8 & 0.3 & 0.0 \\
\hline In percent of public external debt & 17.5 & 22.9 & 26.9 & 25.8 & 20.5 & 12.8 & 5.2 & 0.8 & 0.1 & 0.0 \\
\hline \multicolumn{11}{|l|}{ Obligations to the Fund from existing and } \\
\hline In percent of quota & 15.5 & 15.0 & 12.4 & 34.8 & 115.7 & 172.9 & 161.2 & 92.0 & 15.3 & 2.8 \\
\hline In percent of GDP & 0.3 & 0.3 & 0.3 & 0.7 & 2.2 & 3.0 & 2.6 & 1.4 & 0.2 & 0.0 \\
\hline In percent of exports of goods and nonfactor services & 1.0 & 1.1 & 0.7 & 1.8 & 5.6 & 7.7 & 6.6 & 3.5 & 0.5 & 0.1 \\
\hline In percent of gross reserves & 2.5 & 1.6 & 1.3 & 3.4 & 11.6 & 18.8 & 17.7 & 9.3 & 1.4 & 0.2 \\
\hline In percent of public external debt service & 29.4 & 20.7 & 14.2 & 33.1 & 60.8 & 35.4 & 62.3 & 34.4 & 7.6 & 1.2 \\
\hline
\end{tabular}

Sources: Fund staff estimates and projections.

1/ End of period.

2/ Following the Low Income Countries (LIC) reforms, effective January 7 2010, the PRGF arrangements were renamed Extended Credit Facility (ECF) arrangements.

3/ Repayment schedule based on repurchase obligations and GRA charges. 
Table 9. Georgia: Schedule of Prospective Reviews and Purchases 1/

\begin{tabular}{|c|c|c|c|}
\hline \multirow[t]{2}{*}{ Availability Date } & \multirow[t]{2}{*}{ Condition } & \multicolumn{2}{|c|}{ Available Purchases } \\
\hline & & (In millions of SDR) & (In percent of quota) \\
\hline $15-S e p-08$ & & 161.7 & 107.6 \\
\hline 15-Dec-08 & ormance criteria and other relevant performance criteria 2/ & 63.1 & 42.0 \\
\hline 23-Mar-09 & erformance criteria and other relevant performance criteria $2 /$ & 63.1 & 42.0 \\
\hline 6-Aug-09 & ce criteria and other relevant performance criteria & 94.6 & 62.9 \\
\hline 15-Nov-09 & iformance criteria and other relevant performance criteria & 47.3 & 31.5 \\
\hline 15-Feb-10 & rmance criteria and other relevant performance criteria & 97.3 & 64.7 \\
\hline 9-Jul-10 & nce criteria and other relevant performance criteria & 50.0 & 33.3 \\
\hline $15-$ Nov-10 & ember 2010 performance criteria and other relevant performance criteria & 100.0 & 66.5 \\
\hline 15-Feb-11 & ormance criteria and other relevant performance criteria & 35.0 & 23.3 \\
\hline 15-May-11 & ance criteria and other relevant performance criteria & 35.0 & 23.3 \\
\hline Total available & & 747.1 & 497.1 \\
\hline
\end{tabular}

1/ Reflects the augmentation of access by SDR270 million and the extended arrangement through June 2011.

2/ As the authorities did not draw the purchase that became available at the time of the first review, SDR 126.2 million were available and purchased at the second review. 
Table 10. Georgia: External Financing Requirements and Sources, 2008-15

(In millions of U.S. dollars)

\begin{tabular}{|c|c|c|c|c|c|c|c|c|}
\hline & 2008 & 2009 & 2010 & 2011 & 2012 & 2013 & 2014 & 2015 \\
\hline Current account deficit & $-2,915$ & $-1,274$ & $-1,355$ & $-1,437$ & $-1,480$ & $-1,469$ & $-1,466$ & $-1,430$ \\
\hline Total sources & 3,278 & 1,796 & 1,764 & 1,850 & 2,140 & 3,094 & 2,754 & 2,676 \\
\hline Capital flows & 3,191 & 2,231 & 1,472 & 1,841 & 2,070 & 2,920 & 2,734 & 2,853 \\
\hline Long-term loan disbursements to public sector & 227 & 427 & 404 & 327 & 345 & 343 & 336 & 339 \\
\hline Other $1 /$ & 482 & 274 & 0 & 0 & 0 & 500 & 0 & 250 \\
\hline Private sector & 2,396 & 1,362 & 843 & 1,432 & 1,665 & 2,027 & 2,357 & 2,225 \\
\hline Foreign direct investment in Georgia & 1,564 & 764 & 694 & 888 & 1,007 & 1,144 & 1,299 & 1,405 \\
\hline Long-term loan disbursements to private sector & 757 & 545 & 243 & 390 & 538 & 876 & 1,020 & 747 \\
\hline Advance Repayments & -29 & -194 & 0 & 0 & 0 & 0 & 0 & 0 \\
\hline Change in reserves (- increase) & -131 & -616 & -154 & -95 & 70 & 174 & 20 & -177 \\
\hline \multicolumn{9}{|l|}{ Memorandum items (in percent of GDP): } \\
\hline Total financing requirements & -25.5 & -16.7 & -16.4 & -17.0 & -18.2 & -24.3 & -20.0 & -18.0 \\
\hline Total sources & 25.5 & 16.7 & 16.4 & 17.0 & 18.2 & 24.3 & 20.0 & 18.0 \\
\hline Capital inflows & 24.8 & 20.8 & 13.7 & 16.9 & 17.6 & 23.0 & 19.9 & 19.2 \\
\hline Exceptional financing & 1.7 & 1.7 & 4.2 & 1.0 & 0.0 & 0.0 & 0.0 & 0.0 \\
\hline Change in reserves (- increase) & -1.0 & -5.7 & -1.4 & -0.9 & 0.6 & 1.4 & 0.1 & -1.2 \\
\hline
\end{tabular}

Sources: Georgian Statistics Department; National Bank of Georgia; and Fund staff estimates.

$1 /$ Including the receipts and the repayment of the Eurobond-2013.

2/ Including errors and omissions.

3/ ECF (formerly known as PRGF) disbursements in 2006 and 2007, SBA purchases from 2008 on, including augmentation in 2010-11. 
Table 11. Georgia: Public External Debt, 2008-09

\begin{tabular}{lrrrrrr}
\hline & Dec-08 & Jun-09 & Dec-09 & Dec-08 & Jun-09 & Dec-09 \\
\hline & \multicolumn{2}{c}{ (In millions of U.S. dollars) } & \multicolumn{3}{c}{ (In percent of GDP) } \\
\cline { 2 - 7 } & & & & & & \\
Total & 2,691 & 2,925 & 3,382 & 20.9 & 27.2 & 31.5 \\
Multilateral & 1,648 & 1,879 & 2,321 & 12.8 & 17.5 & 21.6 \\
WB - IDA & 988 & 1,016 & 1,252 & 7.7 & 9.5 & 11.7 \\
IMF & 465 & 647 & 786 & 3.6 & 6.0 & 7.3 \\
Other & 195 & 216 & 282 & 1.5 & 2.0 & 2.6 \\
Bilateral & 535 & 538 & 560 & 4.2 & 5.0 & 5.2 \\
Paris Club & 446 & 412 & 466 & 3.5 & 3.8 & 4.3 \\
Non-Paris Club & 89 & 127 & 94 & 0.7 & 1.2 & 0.9 \\
Commercial & 508 & 508 & 501 & 3.9 & 4.7 & 4.7 \\
\hline
\end{tabular}

Source: Ministry of Finance of Georgia. 
Table 12. Georgia: Private External Debt, 2008-09

\begin{tabular}{|c|c|c|c|c|c|c|c|c|c|c|}
\hline & Dec-08 & Mar-09 & Jun-09 & Sep-09 & Dec-09 & Dec-08 & Mar-09 & Jun-09 & Sep-09 & Dec-09 \\
\hline & \multicolumn{5}{|c|}{ (In millions of U.S. dollars) } & \multicolumn{5}{|c|}{ (In percent of GDP) } \\
\hline Total Private debt $1 /$ & 2,829 & 2,757 & 2,763 & 2,763 & 2,893 & 22.0 & 21.4 & 21.5 & 21.5 & 22.5 \\
\hline Banks & 1,684 & 1,656 & 1,621 & 1,549 & 1,601 & 13.1 & 12.9 & 12.6 & 12.0 & 12.4 \\
\hline Short-term & 566 & 404 & 390 & 361 & 441 & 4.4 & 3.1 & 3.0 & 2.8 & 3.4 \\
\hline Of which: Loans & 355 & 214 & 200 & 140 & 104 & 2.8 & 1.7 & 1.6 & 1.1 & 0.8 \\
\hline Of which: Currency and deposits 2/ & 209 & 187 & 186 & 220 & 336 & 1.6 & 1.5 & 1.4 & 1.7 & 2.6 \\
\hline Long-term & 1,308 & 1,332 & 1,231 & 1,189 & 1,161 & 10.2 & 10.4 & 9.6 & 9.2 & 9.0 \\
\hline Of which: Loans & 1,308 & 1,332 & 1,231 & 1,189 & 1,161 & 10.2 & 10.4 & 9.6 & 9.2 & 9.0 \\
\hline Other Sectors & 1,146 & 1,102 & 1,142 & 1,214 & 1,291 & 8.9 & 8.6 & 8.9 & 9.4 & 10.0 \\
\hline Short-term & 389 & 361 & 403 & 434 & 448 & 3.0 & 2.8 & 3.1 & 3.4 & 3.5 \\
\hline Of which: Trade credits & 324 & 292 & 315 & 324 & 337 & 2.5 & 2.3 & 2.4 & 2.5 & 2.6 \\
\hline Long-term & 757 & 740 & 739 & 780 & 844 & 5.9 & 5.8 & 5.7 & 6.1 & 6.6 \\
\hline Of which: Loans & 757 & 740 & 739 & 780 & 844 & 5.9 & 5.8 & 5.7 & 6.1 & 6.6 \\
\hline Direct Investment: Intercompany Lending & 1,992 & 2,019 & 2,044 & 2,049 & 2,087 & 15.5 & 15.7 & 15.9 & 15.9 & 16.2 \\
\hline
\end{tabular}

Source: National Bank of Georgia.

1/ Excluding intercompany debt from foreign direct investors.

2/ In line with the recommendations of the Debt Statistics Manual that all currency and deposits be included in the short-term category unless detailed information is available to make short-term/long-term attribution. 
Table 13a. Georgia: External Debt Sustainability Framework, Baseline Scenario, 2007-2030 1/

(In percent of GDP, unless otherwise indicated)

\begin{tabular}{|c|c|c|c|c|c|c|c|c|c|c|c|c|c|c|c|}
\hline & \multicolumn{3}{|c|}{ Actual } & \multirow{2}{*}{\multicolumn{2}{|c|}{$\begin{array}{l}\text { Historical } 0 \text { Standard } \\
\text { Average } 0 \text { Deviation }\end{array}$}} & \multicolumn{6}{|c|}{ Projections } & \multirow[b]{2}{*}{$\begin{array}{c}2010-2015 \\
\text { Average }\end{array}$} & \multirow[b]{2}{*}{2020} & \multirow[b]{2}{*}{2030} & \multirow[b]{2}{*}{$\begin{array}{c}2016-2031 \\
\text { Average }\end{array}$} \\
\hline & 2007 & 2008 & 2009 & & & 2010 & 2011 & 2012 & 2013 & 2014 & 2015 & & & & \\
\hline External debt (nominal) 1/ & 38.6 & 44.4 & 58.4 & & & 68.8 & 73.0 & 69.0 & 64.5 & 61.2 & 57.7 & & 49.9 & 34.5 & \\
\hline $\mathrm{o} / \mathrm{w}$ public and publicly guaranteed $(\mathrm{PPG})$ & 17.5 & 20.9 & 31.5 & & & 40.6 & 43.2 & 40.5 & 37.0 & 34.1 & 32.7 & & 24.9 & 15.5 & \\
\hline Change in external debt & 4.0 & 5.8 & 14.0 & & & 10.4 & 4.2 & -4.0 & -4.5 & -3.3 & -3.6 & & -1.3 & -1.4 & \\
\hline Identified net debt-creating flows & -4.8 & 3.3 & 14.1 & & & 4.1 & 2.8 & 1.2 & -0.2 & -1.2 & -2.1 & & 2.0 & 6.7 & \\
\hline Non-interest current account deficit & 18.2 & 20.4 & 8.9 & 9.0 & 7.0 & 9.1 & 9.5 & 8.9 & 7.9 & 7.3 & 6.4 & & 9.5 & 12.7 & 10.4 \\
\hline Deficit in balance of goods and services & 26.7 & 29.6 & 19.2 & & & 20.3 & 19.8 & 18.4 & 16.6 & 15.6 & 14.4 & & 15.6 & 16.2 & \\
\hline Exports & 31.1 & 28.7 & 29.8 & & & 35.8 & 39.1 & 39.4 & 39.7 & 39.9 & 40.2 & & 36.5 & 31.9 & \\
\hline Imports & 57.9 & 58.3 & 49.0 & & & 56.1 & 59.0 & 57.8 & 56.3 & 55.6 & 54.7 & & 52.1 & 48.1 & \\
\hline Net current transfers (negative $=$ inflow) & -6.7 & -8.2 & -8.9 & -5.5 & 3.2 & -10.4 & -9.5 & -8.7 & -8.2 & -7.9 & -7.6 & & -6.3 & -4.4 & -5.7 \\
\hline $\mathrm{o} / \mathrm{w}$ official & -1.4 & -2.8 & -1.2 & & & -2.1 & -1.1 & -0.7 & -0.4 & -0.4 & -0.4 & & -0.2 & -0.1 & \\
\hline Other current account flows (negative = net inflow) & -1.9 & -1.0 & -1.5 & & & -0.9 & -0.9 & -0.8 & -0.6 & -0.5 & -0.5 & & 0.2 & 0.8 & \\
\hline Net FDI (negative = inflow) & -16.4 & -11.8 & -7.1 & -8.1 & 5.6 & -6.5 & -8.2 & -8.6 & -9.0 & -9.3 & -9.3 & & -8.3 & -6.7 & -7.9 \\
\hline Endogenous debt dynamics $2 /$ & -6.5 & -5.3 & 12.3 & & & 1.5 & 1.5 & 0.8 & 1.0 & 0.8 & 0.8 & & 0.9 & 0.8 & \\
\hline Contribution from nominal interest rate & 1.8 & 2.7 & 3.5 & & & 4.1 & 4.3 & 4.2 & 4.1 & 3.8 & 3.6 & & 3.3 & 2.5 & \\
\hline Contribution from real GDP growth & -3.2 & -0.7 & 2.1 & & & -2.6 & -2.7 & -3.4 & -3.2 & -3.0 & -2.8 & & -2.4 & -1.7 & \\
\hline Contribution from price and exchange rate changes & -5.1 & -7.2 & 6.7 & & & & & & & & & & & & \\
\hline Residual (3-4) 3/ & 8.8 & 2.5 & -0.1 & & & 6.3 & 1.3 & -5.2 & -4.3 & -2.1 & -1.5 & & -3.4 & -8.1 & \\
\hline $\mathrm{o} / \mathrm{w}$ exceptional financing & 0.3 & 0.3 & 1.5 & & & 0.0 & 0.0 & 0.0 & 0.0 & 0.0 & 0.0 & & 0.0 & 0.0 & \\
\hline $\mathrm{PV}$ of external debt $4 /$ & $\ldots$ & $\ldots$ & 52.7 & & & 60.2 & 64.2 & 61.6 & 59.5 & 58.3 & 56.7 & & 49.5 & 35.3 & \\
\hline In percent of exports & $\ldots$ & $\ldots$ & 177.2 & & & 168.1 & 163.9 & 156.5 & 150.0 & 146.0 & 141.0 & & 135.5 & 110.8 & \\
\hline PV of PPG external debt & $\ldots$ & $\ldots$ & 25.8 & & & 32.0 & 34.4 & 33.1 & 32.0 & 31.2 & 31.7 & & 24.5 & 16.3 & \\
\hline In percent of exports & $\ldots$ & $\ldots$ & 86.7 & & & 89.4 & 87.8 & 84.1 & 80.7 & 78.1 & 78.8 & & 67.1 & 51.2 & \\
\hline In percent of government revenues & ... & $\ldots$ & 95.1 & & & 118.8 & 124.3 & 120.3 & 116.8 & 113.8 & 115.8 & & 89.4 & 59.6 & \\
\hline Debt service-to-exports ratio (in percent) & 13.2 & 17.7 & 26.2 & & & 20.5 & 19.1 & 22.3 & 38.5 & 27.0 & 25.0 & & 27.4 & 26.0 & \\
\hline PPG debt service-to-exports ratio (in percent) & 3.5 & 3.4 & 5.2 & & & 5.2 & 5.4 & 7.9 & 18.8 & 5.6 & 6.2 & & 6.4 & 6.1 & \\
\hline PPG debt service-to-revenue ratio (in percent) & 3.8 & 3.6 & 5.7 & & & 6.9 & 7.6 & 11.3 & 27.2 & 8.1 & 9.1 & & 8.6 & 7.2 & \\
\hline Total gross financing need (Billions of U.S. dollars) & 1.0 & 2.4 & 2.0 & & & 2.0 & 1.9 & 2.1 & 2.7 & 2.1 & 2.0 & & 3.4 & 8.0 & \\
\hline Non-interest current account deficit that stabilizes debt ratio & 14.2 & 14.6 & -5.1 & & & -1.3 & 5.3 & 12.9 & 12.4 & 10.5 & 10.0 & & 10.8 & 14.0 & \\
\hline \multicolumn{16}{|l|}{ Key macroeconomic assumptions } \\
\hline Real GDP growth (in percent) & 12.3 & 2.3 & -3.9 & 5.9 & 5.0 & 4.5 & 4.0 & 5.0 & 5.0 & 5.0 & 5.0 & 4.8 & 5.0 & 5.0 & 5.0 \\
\hline GDP deflator in US dollar terms (change in percent) & 17.2 & 23.0 & -13.1 & 8.9 & 11.7 & -4.3 & -2.6 & 2.9 & 3.0 & 3.0 & 3.0 & 0.8 & 3.0 & 3.0 & 3.0 \\
\hline Effective interest rate (percent) $5 /$ & 6.7 & 8.7 & 6.7 & 5.0 & 2.3 & 7.0 & 6.3 & 6.2 & 6.5 & 6.4 & 6.4 & 6.5 & 6.9 & 7.4 & 7.0 \\
\hline Growth of exports of G\&S (US dollar terms, in percent) & 24.7 & 15.9 & -13.3 & 18.9 & 15.4 & 20.2 & 10.8 & 8.7 & 8.9 & 8.9 & 8.9 & 11.1 & 6.3 & 7.1 & 6.5 \\
\hline Growth of imports of G\&S (US dollar terms, in percent) & 34.1 & 26.7 & -29.8 & 22.7 & 23.3 & 14.5 & 6.5 & 5.8 & 5.4 & 6.7 & 6.4 & 7.6 & 7.2 & 7.3 & 7.2 \\
\hline Grant element of new public sector borrowing (in percent) & & & & $\ldots$ & $\ldots$ & 27.3 & 27.2 & 24.3 & 2.8 & 22.6 & 8.4 & 18.8 & 12.6 & 8.0 & 12.2 \\
\hline Government revenues (excluding grants, in percent of GDP) & 28.7 & 27.5 & 27.2 & & & 27.0 & 27.7 & 27.5 & 27.4 & 27.4 & 27.4 & & 27.4 & 27.4 & 27.4 \\
\hline Aid flows (in Billions of US dollars) $7 /$ & 0.2 & 0.6 & 0.7 & & & 0.7 & 0.5 & 0.4 & 0.4 & 0.4 & 0.4 & & 0.4 & 0.4 & \\
\hline $\mathrm{o} / \mathrm{w}$ Grants & 0.1 & 0.4 & 0.2 & & & 0.3 & 0.2 & 0.1 & 0.1 & 0.1 & 0.1 & & 0.1 & 0.0 & \\
\hline $\mathrm{o} / \mathrm{w}$ Concessional loans & 0.2 & 0.2 & 0.4 & & & 0.4 & 0.3 & 0.3 & 0.3 & 0.3 & 0.3 & & 0.4 & 0.4 & \\
\hline Grant-equivalent financing (in percent of GDP) $8 /$ & $\ldots$ & $\ldots$ & $\ldots$ & & & 5.4 & 2.5 & 1.5 & 0.8 & 1.1 & 0.8 & & 0.6 & 0.3 & 0.5 \\
\hline Grant-equivalent financing (in percent of external financing) $8 /$ & $\ldots$ & $\ldots$ & $\ldots$ & & & 44.7 & 45.3 & 37.7 & 10.3 & 33.5 & 16.5 & & 20.3 & 12.7 & 19.1 \\
\hline \multicolumn{16}{|l|}{ Memorandum items: } \\
\hline Nominal GDP (Billions of US dollars) & 10.2 & 12.9 & 10.7 & & & 10.7 & 10.9 & 11.8 & 12.7 & 13.8 & 14.9 & & 22.0 & 48.2 & \\
\hline Nominal dollar GDP growth & 31.6 & 25.9 & -16.5 & & & 0.0 & 1.3 & 8.0 & 8.2 & 8.2 & 8.2 & 5.6 & 8.2 & 8.1 & 8.2 \\
\hline PV of PPG external debt (in Billions of US dollars) & & & 2.8 & & & 3.4 & 3.7 & 3.9 & 4.1 & 4.3 & 4.7 & & 5.4 & 7.9 & \\
\hline (PVt-PVt-1)/GDPt-1 (in percent) & & & & & & 6.2 & 2.8 & 1.4 & 1.5 & 1.7 & 3.1 & 2.8 & 1.1 & 0.5 & 0.8 \\
\hline Gross remittances (Billions of US dollars) & 0.2 & 0.3 & 0.3 & & & 0.3 & 0.3 & 0.4 & 0.4 & 0.4 & 0.4 & & 0.5 & 0.9 & \\
\hline $\mathrm{PV}$ of PPG external debt (in percent of GDP $+\mathrm{r}$ & $\ldots$ & $\ldots$ & 25.1 & & & 31.0 & 33.3 & 32.1 & 31.1 & 30.3 & 30.8 & & 23.9 & 16.0 & \\
\hline PV of PPG external debt (in percent of exports + remittan & $\ldots$ & $\ldots$ & 78.9 & & & 82.2 & 81.3 & 78.0 & 75.0 & 72.8 & 73.6 & & 62.8 & 48.4 & \\
\hline Debt service of PPG external debt (in percent of exports + remittances) & $\ldots$ & $\ldots$ & 4.8 & & & 4.7 & 5.0 & 7.3 & 17.5 & 5.2 & 5.8 & & 6.0 & 5.8 & \\
\hline
\end{tabular}

Sources: Country authorities: and stefestimates and pojerol

1/Includes both public and private sector external debt.

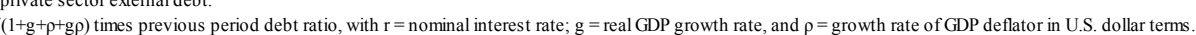

3/ Includes exceptional financing (i.e., changes in arrears and debt relief); changes in gross foreign assets; and valuation adjustments. For projections also includes contribution from price and exchange rate changes.

4/ Assumes that PV of private sector debt is equivalent to its face value.

6/ Historical averages and standard deviations are generally derived over the past 10 years, subject to data availability.

7/ Defined as grants, concessional loans, and debt relief. 
Table 13b.Georgia: Sensitivity Analysis for Key Indicators of Public and Publicly Guaranteed External Debt, 2010-2030 (In percent)

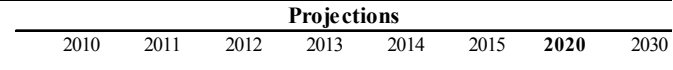

PV of de bt-to GDP ratio

Baseline

A. Alternative Scenarios

A1. Key variables at their historical averages in 2010-2030 1/

A2. New public sector loans on less favorable terms in 2010-2030 2

\section{B. Bound Tests}

B1. Real GDP growth at historical average minus one standard deviation in 2011-2012

B2. Export value growth at historical average minus one standard deviation in 2011-2012

B4. Net non-debt

B5. Combination of B1-B4 using one-half standard deviation shock

B6. One-time 30 percent nominal depreciation relative to the baseline in 20115 /

\section{PV of debt-to-exports ratio}

Baseline

A. Alternative Scenarios

A1. Key variables at their historical averages in 2010-2030 1 -

A2. New public sector loans on less favorable terms in 2010-2030 2

\section{B. Bound Test}

B1. Real GDP growth at historical average minus one standard deviation in 2011-2012

B2. Export value growth at historical average minus one standard deviation in 2011-2012 3/

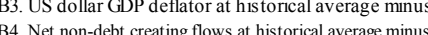

B5. Combination of B1-B4 using one-half standard deviation shocks

B6. One-time 30 percent nominal depreciation relative to the baseline in 20115

\section{PV of debt-to-revenue ratio}

Baseline

\section{A. Alternative Scenarios}

A1. Key variables at their historical averages in 2010-2030

A2. New public sector loans on less favorable terms in 2010-2030 2

\section{B. Bound Tests}

B1. Real GDP growth at historical average minus one standard deviation in 2011-2012

B2. Export value growth at historical average minus one standard deviation in 2011-2012 3 B3. US dollar GDP deflator at historical average minus one standard deviation in 2011-20 B4. Net non-debt creating flows at historical average minus one standard deviation in 2011-2012 B5. Combination of Bl-B4 using one-half standard deviation shock B6. One-time 30 percent nominal depreciation relative to the baseline in $20115 /$

$\begin{array}{llllllll}32 & 34 & 33 & 32 & 31 & 32 & \mathbf{2 4} & 16 \\ & & & & & & & \\ 32 & 29 & 26 & 25 & 24 & 26 & \mathbf{1 7} & -22 \\ 32 & 35 & 35 & 34 & 34 & 35 & \mathbf{3 0} & 25\end{array}$

$\begin{array}{llllllll}32 & 35 & 34 & 33 & 32 & 32 & \mathbf{2 3} & 15 \\ 32 & 36 & 39 & 37 & 35 & 35 & \mathbf{2 5} & 14 \\ 32 & 34 & 34 & 32 & 31 & 31 & \mathbf{2 3} & 15 \\ 32 & 46 & 55 & 53 & 51 & 50 & \mathbf{3 5} & 16 \\ 32 & 39 & 44 & 42 & 40 & 40 & \mathbf{2 8} & 14 \\ 32 & 49 & 46 & 44 & 43 & 43 & \mathbf{3 1} & 20\end{array}$

$\begin{array}{llllllll}89 & 88 & 84 & 81 & 78 & 79 & \mathbf{6 7} & 5\end{array}$

$\begin{array}{llllllll}89 & 75 & 67 & 62 & 61 & 64 & \mathbf{4 6} & -68 \\ 89 & 90 & 88 & 85 & 85 & 87 & \mathbf{8 2} & 79\end{array}$

$\begin{array}{rrrrrrrr}119 & 124 & 120 & 117 & 114 & 116 & \mathbf{8 9} & 60 \\ & & & & & & & \\ 119 & 107 & 95 & 90 & 89 & 94 & \mathbf{6 1} & -79 \\ 119 & 127 & 126 & 123 & 124 & 127 & \mathbf{1 1 0} & 92 \\ & & & & & & & \\ & & & & & & & \\ 119 & 126 & 125 & 120 & 115 & 116 & \mathbf{8 4} & 54 \\ 119 & 131 & 140 & 134 & 129 & 129 & \mathbf{9 3} & 53 \\ 119 & 123 & 124 & 118 & 114 & 115 & \mathbf{8 3} & 53 \\ 119 & 166 & 201 & 193 & 185 & 183 & \mathbf{1 2 9} & 58 \\ 119 & 141 & 159 & 152 & 146 & 146 & \mathbf{1 0 4} & 53 \\ 119 & 177 & 169 & 161 & 155 & 157 & \mathbf{1 1 4} & 73\end{array}$


Table 13b.Georgia: Sensitivity Analysis for Key Indicators of Public and Publicly Guaranteed External Debt, 2010-2030 (continued) (In percent)

\section{Debt service-to-exports ratio}

Baseline

\section{A. Alternative Scenarios}

A1. Key variables at their historical averages in 2010-2030 1/

A2. New public sector loans on less favorable terms in 2010-2030 2

\section{B. Bound Tests}

B1. Real GDP growth at historical average minus one standard deviation in 2011-2012

B2. Export value growth at historical average minus one standard deviation in 2011-2012 3/

B3. US dollar GDP deflator at historical average minus one standard deviation in 2011-2012

B4. Net non-debt creating flows at historical average minus one standard deviation in 2011-2012 4 /

B5. Combination of B1-B4 using one-half standard deviation shocks

B6. One-time 30 percent nominal depreciation relative to the baseline in 20115 /

Debt service-to-revenue ratio

Baseline

\section{A. Alternative Scenarios}

A1. Key variables at their historical averages in 2010-2030 1/

A2. New public sector loans on less favorable terms in 2010-2030 2

\section{B. Bound Tests}

B1. Real GDP growth at historical average minus one standard deviation in 2011-2012

B2. Export value growth at historical average minus one standard deviation in 2011-2012 3/

B3. US dollar GDP deflator at historical average minus one standard deviation in 2011-2012

B4. Net non-debt creating flows at historical average minus one standard deviation in 2011-2012

B5. Combination of B1-B4 using one-half standard deviation shocks

B6. One-time 30 percent nominal depreciation relative to the baseline in 2011 5/

Memorandum item

Grant element assumed on residual financing (i.e., financing required above baseline) 6/

$5 \quad 5$

8

10

$\begin{array}{rrrrr}14 & 4 & 4 & \mathbf{4} & -1 \\ 18 & 5 & 4 & \mathbf{6} & 7\end{array}$

Sources: Country authorities; and staff estimates and projections.

1/ Variables include real GDP growth, growth of GDP deflator (in U.S. dollar terms), non-interest current account in percent of GDP, and non-debt creating flows .

2/ Assumes that the interest rate on new borrowing is by 2 percentage points higher than in the baseline., while grace and maturity periods are the same as in the baseline.

3 / Exports values are assumed to remain permanently at the lower level, but the current account as a share of GDP is assumed to return to its baseline level after the shock (implicitly assuming an offsetting adjustment in import levels).

4/ Includes official and private transfers and FDI

5/ Depreciation is defined as percentage decline in dollar/local currency rate, such that it never exceeds 100 percent.

6/ Applies to all stress scenarios except for A2 (less favorable financing) in which the terms on all new financing are as specified in footnote 2. 
Table 14a.Georgia: Public Sector Debt Sustainability Framework, Baseline Scenario, 2007-2030 (In percent of GDP, unless otherwise indicated)

\begin{tabular}{|c|c|c|c|c|c|c|c|c|c|c|c|c|c|c|c|}
\hline & \multicolumn{3}{|c|}{ Actual } & \multirow[b]{2}{*}{ Average } & \multirow[b]{2}{*}{$\begin{array}{c}\text { Standard } \\
\text { Deviation } \\
\end{array}$} & \multicolumn{5}{|l|}{ Estimate } & \multicolumn{5}{|c|}{ Projections } \\
\hline & 2007 & 2008 & 2009 & & & 2010 & 2011 & 2012 & 2013 & 2014 & 2015 & $\begin{array}{l}2010-15 \\
\text { Average }\end{array}$ & 2020 & 2030 & $\begin{array}{l}2016-30 \\
\text { Average }\end{array}$ \\
\hline Public sector debt 1/ & 22.3 & 25.0 & 37.1 & & & 46.1 & 49.3 & 47.1 & 45.1 & 42.2 & 40.5 & & 36.1 & 35.5 & \\
\hline $\mathrm{o} / \mathrm{w}$ foreign-currency denominated & 17.5 & 20.9 & 31.5 & & & 40.6 & 43.2 & 40.5 & 37.0 & 34.1 & 32.7 & & 24.9 & 15.5 & \\
\hline Change in public sector debt & -5.6 & 2.7 & 12.1 & & & 9.0 & 3.2 & -2.2 & -1.9 & -2.9 & -1.6 & & -0.4 & 0.0 & \\
\hline Identified debt-creating flows & -6.9 & -1.5 & 11.6 & & & 4.8 & 3.1 & -0.8 & -1.3 & -1.8 & -1.2 & & -0.6 & 0.1 & \\
\hline Primary deficit & 4.1 & 5.6 & 8.2 & 1.4 & 3.6 & 5.0 & 3.3 & 1.8 & 0.7 & 0.3 & 0.4 & 1.9 & 0.6 & 0.7 & 0.6 \\
\hline Revenue and grants & 29.3 & 30.7 & 29.3 & & & 29.8 & 29.1 & 28.2 & 28.0 & 27.9 & 27.8 & & 27.7 & 27.5 & \\
\hline of which: grants & 0.6 & 3.2 & 2.2 & & & 2.9 & 1.4 & 0.7 & 0.6 & 0.5 & 0.4 & & 0.3 & 0.1 & \\
\hline Primary (noninterest) expenditure & 33.4 & 36.3 & 37.6 & & & 34.9 & 32.3 & 30.1 & 28.7 & 28.2 & 28.2 & & 28.2 & 28.2 & \\
\hline Automatic debt dynamics & -5.8 & -3.4 & 5.4 & & & 0.8 & 0.6 & -2.2 & -1.9 & -1.9 & -1.6 & & -1.2 & -0.6 & \\
\hline Contribution from interest rate/growth differential & -3.4 & -0.7 & 2.1 & & & -1.1 & -0.9 & -1.7 & -1.5 & -1.5 & -1.3 & & -0.9 & -0.4 & \\
\hline of which: contribution from average real interest rate & -0.4 & -0.2 & 1.0 & & & 0.5 & 0.8 & 0.6 & 0.7 & 0.6 & 0.7 & & 0.9 & 1.3 & \\
\hline of which: contribution from real GDP growth & -3.1 & -0.5 & 1.0 & & & -1.6 & -1.8 & -2.3 & -2.2 & -2.1 & -2.0 & & -1.7 & -1.7 & \\
\hline Contribution from real exchange rate depreciation & -2.3 & -2.7 & 3.3 & & & 1.9 & 1.5 & -0.5 & -0.4 & -0.4 & -0.3 & & $\ldots$ & ... & \\
\hline Other identified debt-creating flows & -5.2 & -3.7 & -2.0 & & & -1.1 & -0.7 & -0.4 & -0.2 & -0.2 & 0.0 & & 0.0 & 0.0 & \\
\hline Privatization receipts (negative) & -5.2 & -3.7 & -2.0 & & & -1.1 & -0.7 & -0.4 & -0.2 & -0.2 & 0.0 & & 0.0 & 0.0 & \\
\hline Recognition of implicit or contingent liabilities & 0.0 & 0.0 & 0.0 & & & 0.0 & 0.0 & 0.0 & 0.0 & 0.0 & 0.0 & & 0.0 & 0.0 & \\
\hline Debt relief(HIPC and other) & 0.0 & 0.0 & 0.0 & & & 0.0 & 0.0 & 0.0 & 0.0 & 0.0 & 0.0 & & 0.0 & 0.0 & \\
\hline Other (specify, e.g. bank recapitalization) & 0.0 & 0.0 & 0.0 & & & 0.0 & 0.0 & 0.0 & 0.0 & 0.0 & 0.0 & & 0.0 & 0.0 & \\
\hline Residual, including asset changes & 1.3 & 4.2 & 0.5 & & & 4.2 & 0.1 & -1.5 & -0.6 & -1.2 & -0.4 & & 0.2 & -0.1 & \\
\hline \multicolumn{16}{|l|}{ Other Sustainability Indicators } \\
\hline PV of public sector debt & 4.8 & 4.1 & 31.4 & & & 37.5 & 40.5 & 39.7 & 40.1 & 39.3 & 39.6 & & 35.7 & 36.3 & \\
\hline $\mathrm{o} / \mathrm{w}$ foreign-currency denominated & 0.0 & 0.0 & 25.8 & & & 32.0 & 34.4 & 33.1 & 32.0 & 31.2 & 31.7 & & 24.5 & 16.3 & \\
\hline $\mathrm{o} / \mathrm{w}$ external & $\ldots$ & $\ldots$ & 25.8 & & & 32.0 & 34.4 & 33.1 & 32.0 & 31.2 & 31.7 & & 24.5 & 16.3 & \\
\hline $\mathrm{PV}$ of contingent liabilities (not included in public sector debt) & $\ldots$ & $\ldots$ & $\ldots$ & & & $\ldots$ & $\ldots$ & $\ldots$ & $\ldots$ & $\ldots$ & $\ldots$ & & $\ldots$ & $\ldots$ & \\
\hline Gross financing need $2 /$ & 5.7 & 7.1 & 10.3 & & & 7.5 & 6.1 & 5.7 & 9.1 & 3.5 & 3.8 & & 4.2 & 4.6 & \\
\hline $\mathrm{PV}$ of public sector debt-to-revenue and grants ratio (in percent) & 16.3 & 13.4 & 107.2 & & & 125.6 & 139.4 & 140.6 & 143.2 & 140.7 & 142.2 & & 129.3 & 132.2 & \\
\hline PV of public sector debt-to-revenue ratio (in percent) & 16.7 & 14.9 & 115.7 & & & 139.0 & 146.3 & 144.3 & 146.3 & 143.1 & 144.5 & & 130.5 & 132.7 & \\
\hline o/w external $3 /$ & & & 95.1 & & & 118.8 & 124.3 & 120.3 & 116.8 & 113.8 & 115.8 & & 89.4 & 59.6 & \\
\hline Debt service-to-revenue and grants ratio (in percent) 4/ & 5.5 & 4.7 & 7.1 & & & 8.2 & 9.9 & 13.8 & 29.8 & 11.4 & 12.4 & & 13.0 & 13.9 & \\
\hline Debt service-to-revenue ratio (in percent) $4 /$ & 5.6 & 5.3 & 7.7 & & & 9.1 & 10.4 & 14.2 & 30.5 & 11.6 & 12.6 & & 13.1 & 14.0 & \\
\hline Primary deficit that stabilizes the debt-to-GDP ratio & 9.7 & 2.9 & -3.8 & & & -4.0 & 0.1 & 4.1 & 2.7 & 3.3 & 2.1 & & 1.0 & 0.7 & \\
\hline \multicolumn{16}{|l|}{ Key macroeconomic and fiscal assumptions } \\
\hline Real GDP growth (in percent) & 12.3 & 2.3 & -3.9 & 5.9 & 5.0 & 4.5 & 4.0 & 5.0 & 5.0 & 5.0 & 5.0 & 4.8 & 5.0 & 5.0 & 5.0 \\
\hline Average nominal interest rate on forex debt (in percent) & 1.6 & 3.0 & 2.5 & 2.2 & 0.5 & 2.8 & 2.5 & 2.5 & 2.9 & 2.5 & 2.9 & 2.7 & 2.9 & 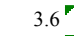 & 3.1 \\
\hline Average real interest rate on domestic debt (in percent) & -2.4 & -2.5 & 9.7 & 1.1 & 5.1 & 3.1 & 5.8 & 5.7 & 6.4 & 5.2 & $5.1^{\mathrm{r}}$ & 5.2 & 5.5 & $5.5^{\circ}$ & 5.6 \\
\hline Real exchange rate depreciation (in percent,+ indicates depreciation) & -12.2 & -15.6 & 14.7 & -5.0 & 9.8 & 6.3 & $\ldots$ & $\ldots$ & $\ldots$ & & $\ldots$ & $\ldots$ & & $\ldots$ & $\ldots$ \\
\hline Inflation rate (GDP deflator, in percent) & 9.7 & 9.7 & -2.0 & 6.4 & 3.8 & 4.8 & 5.0 & 5.0 & 5.0 & 5.0 & 5.0 & 5.0 & 5.0 & 5.0 & 5.0 \\
\hline Growth of real primary spending (deflated by GDP deflator, in percent) & 0.3 & 0.1 & 0.0 & -1.8 & 5.5 & 0.0 & 0.0 & 0.0 & 0.0 & 0.0 & 0.1 & 0.0 & 0.0 & 0.1 & 0.1 \\
\hline Grant element of new external borrowing (in percent) & $\ldots$ & & $\ldots$ & $\ldots$ & $\ldots$ & 27.3 & 27.2 & 24.3 & 2.8 & 22.6 & 8.4 & 18.8 & 12.6 & 8.0 & $\ldots$ \\
\hline
\end{tabular}

Sources: Country authorities; and staff estimates and projections

plus debt service plus the stock of short-term debt at the end of the last period.

Revenues excluding grants.

is defined as the sum of interest and amortization of medium and long-term deb.

$5 /$ Historical averages and standard deviations are generally derived over the past 10 years, subject to data availability. 
Table 14b.Georgia: Sensitivity Analysis for Key Indicators of Public Debt 2010-2030

\begin{tabular}{|c|c|c|c|c|c|c|c|c|}
\hline & \multicolumn{8}{|c|}{ Projections } \\
\hline & 2010 & 2011 & 2012 & 2013 & 2014 & 2015 & 2020 & 2030 \\
\hline \multicolumn{9}{|l|}{ PV of Debt-to-GDP Ratio } \\
\hline Baseline & 37 & 40 & 40 & 40 & 39 & 40 & 36 & 36 \\
\hline \multicolumn{9}{|l|}{ A. Alternative scenarios } \\
\hline A1. Real GDP growth and primary balance are at historical averages & 37 & 38 & 37 & 38 & 38 & 39 & 38 & 41 \\
\hline A2. Primary balance is unchanged from 2010 & 37 & 42 & 45 & 49 & 53 & 57 & 71 & 97 \\
\hline A3. Permanently lower GDP growth $1 /$ & 37 & 41 & 41 & 43 & 44 & 46 & 55 & 99 \\
\hline \multicolumn{9}{|l|}{ B. Bound tests } \\
\hline B1. Real GDP growth is at historical average minus one standard deviations in 2011-2012 & 37 & 43 & 45 & 48 & 49 & 51 & 54 & 67 \\
\hline B2. Primary balance is at historical average minus one standard deviations in 2011-2012 & 37 & 42 & 45 & 45 & 44 & 44 & 39 & 39 \\
\hline B3. Combination of B1-B2 using one half standard deviation shocks & 37 & 41 & 42 & 43 & 43 & 43 & 42 & 46 \\
\hline B4. One-time 30 percent real depreciation in 2011 & 37 & 56 & 55 & 56 & 55 & 56 & 50 & 51 \\
\hline B5. 10 percent of GDP increase in other debt-creating flows in 2011 & 37 & 50 & 49 & 49 & 48 & 48 & 43 & 41 \\
\hline
\end{tabular}

PV of Debt-to-Revenue Ratio 2/

Baseline

\section{A. Alternative scenarios}

A1. Real GDP growth and primary balance are at historical averages

A2. Primary balance is unchanged from 2010

A3. Permanently lower GDP growth 1/

$\begin{array}{llllllll}126 & 131 & 130 & 134 & 135 & 139 & 137 & 148 \\ 126 & 145 & 158 & 175 & 189 & 205 & 257 & 353 \\ 126 & 142 & 147 & 154 & 157 & 165 & 197 & 360\end{array}$

\section{B. Bound tests}

B1. Real GDP growth is at historical average minus one standard deviations in 2011-2012

B2. Primary balance is at historical average minus one standard deviations in 2011-2012

B3. Combination of B1-B2 using one half standard deviation shocks

B4. One-time 30 percent real depreciation in 2011

B5. 10 percent of GDP increase in other debt-creating flows in 2011

Debt Service-to-Revenue Ratio $2 /$

\section{Baseline}

$\begin{array}{llllllll}126 & 146 & 161 & 170 & 174 & 182 & 196 & 242 \\ 126 & 146 & 158 & 160 & 157 & 158 & 143 & 141 \\ 126 & 140 & 148 & 153 & 152 & 156 & 151 & 166 \\ 126 & 194 & 195 & 199 & 198 & 201 & 182 & 184 \\ 126 & 174 & 175 & 176 & 173 & 173 & 155 & 150\end{array}$

\section{A. Alternative scenarios}

A1. Real GDP growth and primary balance are at historical averages

A2. Primary balance is unchanged from 2010

A3. Permanently lower GDP growth 1 /

B. Bound tests

B1. Real GDP growth is at historical average minus one standard deviations in 2011-2012

B2. Primary balance is at historical average minus one standard deviations in 2011-2012

B3. Combination of B1-B2 using one half standard deviation shocks

B4. One-time 30 percent real depreciation in 2011

B5. 10 percent of GDP increase in other debt-creating flows in 2011

$\begin{array}{llllllll}8 & 10 & 13 & 29 & 11 & 12 & 12 & 14 \\ 8 & 10 & 14 & 31 & 13 & 14 & 18 & 27 \\ 8 & 10 & 14 & 31 & 12 & 13 & 16 & 26\end{array}$

Sources: Country authorities; and staff estimates and projections.

1/ Assumes that real GDP growth is at baseline minus one standard deviation divided by the square root of the length of the projection period.

2/ Revenues are defined inclusive of grants. 
Figure 1a. Georgia: Indicators of Public and Publicly Guaranteed External Debt under Alternatives Scenarios, 2010-2030 1/
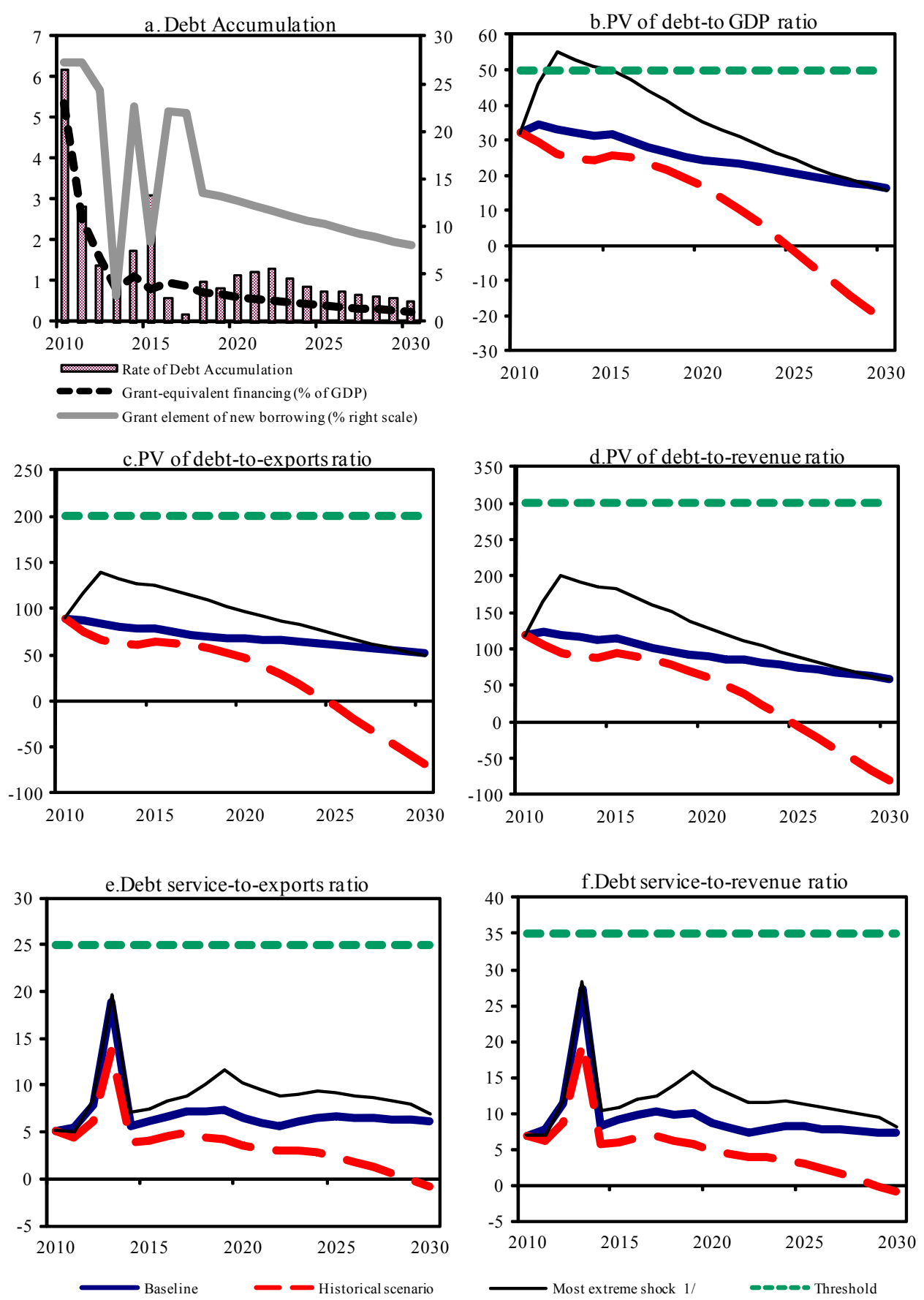

Sources: Country authorities; and staff estimates and projections.

1/ The most extreme stress test is the test that yields the highest ratio in 2020. In figure b. it corresponds to a Non-debt flows shock; in c. to a Non-debt flows shock; in d. to a Non-debt flows shock; in e. to a Non-debt flows shock and in figure f. to a Non-debt flows shock 
Figure 1b.Georgia: Indicators of Public Debt Under Alternative Scenarios, 2010-2030
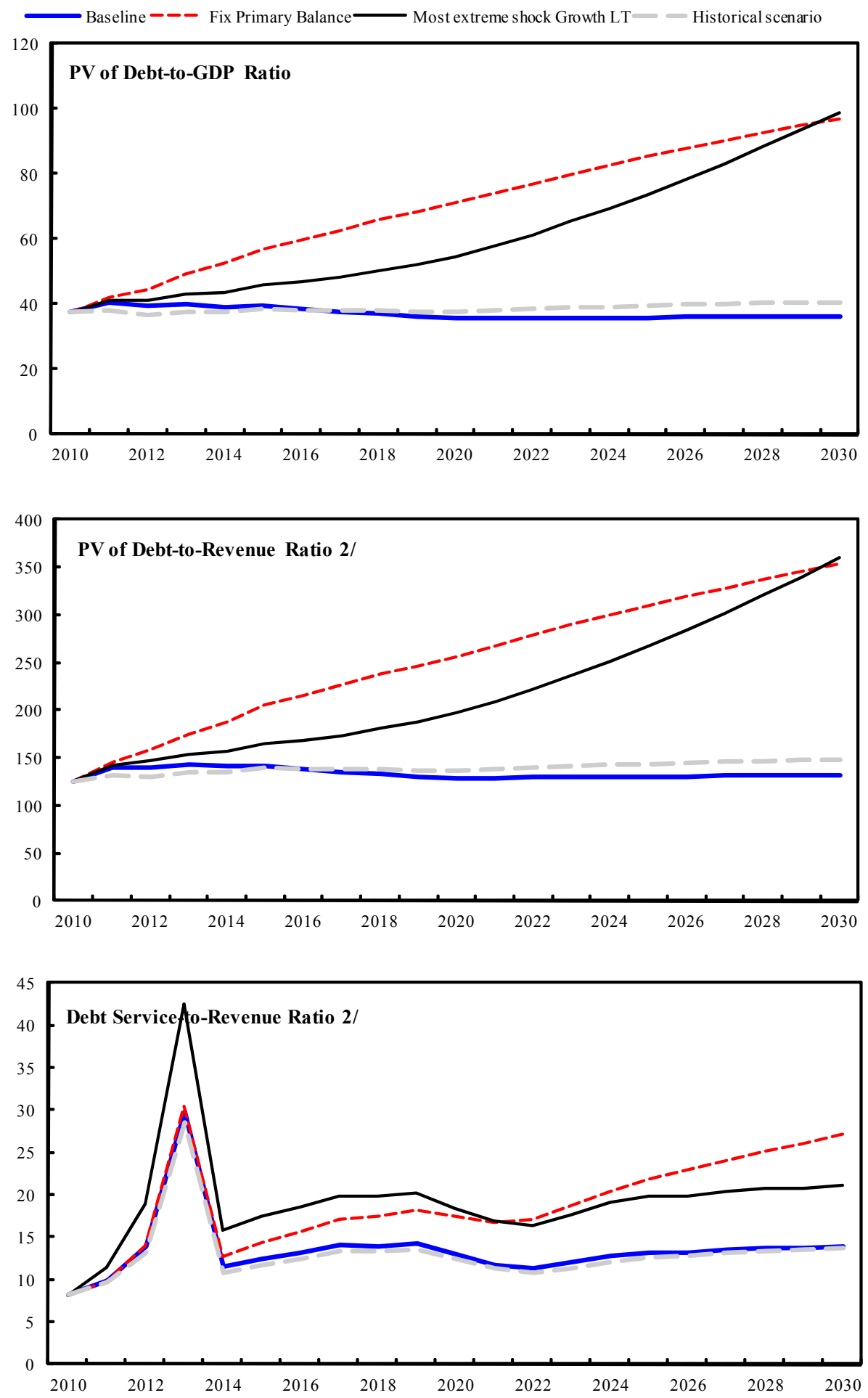

Sources: Country authorities; and staff estimates and projections.

1/ The most extreme stress test is the test that yields the highest ratio in 2020 .

2/ Revenues are defined inclusive of grants. 


\section{ATTACHMENT I. GEORGIA: LETTER OF INTENT}

June 24, 2010

Mr. Dominique Strauss-Kahn

Managing Director

International Monetary Fund

Washington, D.C.

Dear Mr. Strauss-Kahn:

1. In September 2008, the International Monetary Fund (IMF) approved a Stand-By Arrangement (SBA) for Georgia, which was further augmented in August 2009. On March 19, 2010 the IMF's Executive Board completed the fifth review of the SBA allowing for an immediate purchase of an amount equivalent to SDR 97.3 million. We are grateful for the assistance which the IMF provides to Georgia.

2. This letter of intent describes the economic policies that we plan to implement during 2010. As always, we are committed to policies that will maintain macroeconomic stability, protect the economy from shocks associated with the global economic crisis and facilitate the process of macroeconomic adjustment. We, of course, remain committed to implementing the measures contained in previous letters of intent, i.e. those dated September 9, 2008, November 28, 2008, March 10, 2009, July 30, 2009, November 25, 2009, and February 25, 2010.

\section{Recent Economic Developments}

3. Growth in 2009 amounted to minus 3.9 percent. In the fourth quarter Real GDP increased by 0.4 percent, after 5 consecutive quarters of negative growth. Positive year-onyear growth in the fourth quarter was achieved in electricity, gas and water supply, financial intermediation, health care.

4. Balance of payments data for the fourth quarter of 2009 show that exports of goods and services increased year-on-year by 21 percent and imports of goods and services declined by 15 percent, which reflects lower demand for imports. Worker remittances increased 22.1 percent, and FDI inflows declined by 11.2 percent year-on-year. As a result, the current account deficit dropped to 13.4 percent of GDP in the fourth quarter of 2009 , showing slight improvement relative to the same period of the previous year. In 2009 the current account deficit was11.9 percent of GDP compared to 22.7 percent in the previous year. 
5. As a result of a broad decline in economic activity and falling global commodity prices, inflationary pressures have declined. Inflation as of end of 2009 was 3.0 percent, rising to 4 percent in May 2010. Taking into account the recent development of global commodity prices and signs of improvement in economic activity, we expect inflation to stay around 5 percent in 2010 .

6. Fiscal performance in the first quarter of 2010 has been stronger than anticipated. Tax collection in Q1 2010 increased by 13.0 percent y-o-y in nominal terms (excluding one-off payments). Expenditures in the same quarter were contained at GEL 1441.0 million (general government, including capital expenditures), or 1.7 percent lower than in Q1 2009. In all, the Q1 fiscal deficit reached 0.5 percent of the projected annual GDP, which is below the program target. We also expect the deficit through the first half of 2010 to be below the program target.

7. Consistent with our commitment to increase exchange rate flexibility, starting from May 25, 2009, the NBG foreign exchange interventions are conducted only through the foreign exchange auctions. Less frequent interventions have increased market participation, thus dampening one-way speculative pressures. In first quarter, the interventions have relatively increased in the FX market because of seasonal pressures. External gap in private sector is being financed by public sector inflows. Because of the above and seasonal factors the NBG is expecting more interventions in the first half of the year than in the second. In the first quarter of 2010 NBG FX intervention amounted to USD 124 million, which is $38 \%$ less than IMF program incorporates.

8. Seasonal exchange market pressures continued after March. In anticipation that these pressures would be reversed after the May 30 elections, the NBG increased its intervention in April-May to USD 150 million. However, the persistence of pressures points to weakness in FDI inflows in the first half of the year, which has led to a downward revision of projected FDI inflows for the year. The exchange rate (vis-à-vis the USD) has depreciated by 4.6 percent in the first 9 days of June, and by 10.2 cumulatively since the beginning of the year. Intervention volumes remained elevated in the first ten days of June because of the need to stabilize market expectations, but the foreign exchange market has since stabilized.

9. The banking sector continued its positive performance in the first quarter of 2010 . Commercial banks' net profit in the first quarter equaled GEL 15.3 million. Starting from May 2009, the volume of deposits has been growing steadily, posting a 48 percent growth in lari terms by end-May 2010. Bank lending to the economy has picked up, and credit to the economy has increased by nearly 12 percent (4 percent excluding valuation effects) since the beginning of the year (as of June 11). The major part of the increase in credit is attributed to lending in domestic currency. The average capital adequacy was 18.3 at end-March 2010. 
10. In order to enhance the safety and efficiency of the international reserves management process, the NBG is implementing a new portfolio management system. The system will cover front-middle-back office and accounting functionality based on Straight Through Processing (STP) principles. The system will be IFRS compliant and will allow NBG to introduce new, more sophisticated financial instruments and investment techniques in its reserves management process. The new system will help NBG bring its reserves management procedures in line with international best practices. NBG has signed the contract on system supply and implementation with WallstreetSystems, leading portfolio management system supplier who has over 30 central banks clients around the world. The system is expected to become fully operational by the end of 2010 .

11. We have in place a Contingency Plan (CP) which provides a framework for policy makers to coordinate their policies and actions to mitigate systemic risks to the financial sector and, in case of realization of such risks, to reduce the social cost of any ensuing financial distress. Although the banking system is adequately capitalized, the NBG continues to conduct regular stress tests on system wide and bank by bank level. In line with changes in legislation, which enable the supervisor to conduct risk-based supervision, a reorganization process is under way to enable the NBG to use more effectively its resources for risk based supervision. The move toward a risk based framework is done gradually, to minimize regulatory risk for the industry and enable smooth transition towards the new regulatory framework.

12. We continue to improve the efficiency and effectiveness of public finances. The Ministry of Finance is broadly on track in implementing the Public Finance Management Reform Policy Vision 2009-2013. The Reform Action Plan for 2009 has been successfully implemented. The draft budget code was one of our key priorities in 2009. The new budget code was adopted in December 2009, providing a sound framework for fiscal planning and efficiency gains in the area. The new budget code seeks to consolidate all legislation related to the budget process into a single law, to better integrate the medium-term economic and fiscal framework (BDD) and the public investment program into the annual budget cycle, and to prepare the ground for results-oriented budgets.

13. Furthermore, in 2009 we progressed significantly in ensuring functionality of the risk assessment tax audit system, streamlining tax and customs codes and developing necessary sub-legislation, establishing electronic information exchange system between banks and the Revenues Service, improving capital budget document forms, establishing a customs audit and progressing towards risk-based customs control, fostering full functionality of the electronic treasury system and inclusion of all taxes into the e-filing system. The implementation of these reforms has enhanced efficiency and effectiveness in the use of public financial resources and shall contribute to the overall resilience of the economy both now, in a time of stress, and over the medium term. 


\section{Macroeconomic Policies for 2010}

14. Our major macroeconomic challenge continues to be the restoration of economic growth while promoting balance of payments adjustment. This will, of course, require the resumption of private capital inflows and domestic lending in support of investment projects. We recognize that many of the extraordinary actions undertaken in response to the economic crisis are not sustainable in the medium term. Hence, our efforts to enhance macroeconomic stability will concentrate on intensifying economic reforms, and achieving a sustainable fiscal and external balance as quickly as possible.

15. Economic decline in 2009 amounted to 3.9 percent. Based on available economic data, we anticipate that real economy will increase by 2 percent in annual terms in the first quarter of 2010. Overall in the 2010 real GDP growth is projected at $4 \frac{1}{2}$ percent per annum.

16. The current account deficit for 2010 is expected to be around 12.6 percent of GDP. Exports and imports of goods and services are projected to increase by 20 percent and $14 \frac{1}{2}$ percent, respectively. Worker remittances are expected to increase by around 10 percent for the year. Conservatively, we project FDI inflows of just under US\$700 million in 2010, somewhat lower than in 2009. Overall, gross reserves in 2010 are expected to increase by about US\$100 million.

17. We expect a recovery in private capital inflows to begin in the second half of 2010 . Despite considerable fiscal adjustment, the current account deficit is expected to widen marginally in 2010, owing to the economic recovery. In view of the continued high uncertainty in the external environment, there is a need to increase the reserve buffer to more comfortable levels. Accordingly, we have sought the Fund's continued support in covering a balance of payments gap of around US\$450 million in 2010.

18. We consider that restoring a sound fiscal position is critical for the sustainability of the recovery and also to preserve the stability of Georgia's external accounts. We are therefore committed to an ambitious program of deficit reduction. Based on further expenditure containment, an expected recovery of tax revenues owing to the expected rebound in the GDP growth, as well as new revenue measures to be taken in 2010 and 2011, we are committed to steady reductions in the deficit to 2-3 percent of GDP by 2013. In order to enhance fiscal policy flexibility, the proposed constitutional amendment which introduces a referendum requirement for new taxes would become effective only once the deficit has been brought down below our medium-term deficit ceiling of 3 percent of GDP.

19. Our target is to reduce the deficit to 6.3 percent of GDP in 2010 (SBA definition), well below the 7.4 percent deficit target agreed at the time of the Fifth Review of the program. The revenue gain from the upward revision in GDP growth and an increase in excises coming into effect on August 1, 2010 (with an estimated yield of 0.3 percent of GDP) 
will enable us to meet this more ambitious deficit target as well as some urgent spending priorities. The supplementary budget consistent with the new level of spending was submitted to Parliament on June 4, and we expect it to be approved by the end of June 2010 . The additional revenue measures will be adopted in July based on a revision of the existing tax code. We also intend to cap government spending through the rest of the year at the level of the supplementary budget, so as to ensure that any additional revenue gains would go toward faster deficit reduction. Furthermore, as part of the new tax and customs code, we intend to introduce a package of new revenue measures effective January 1, 2011, which include harmonization of income tax rates and partial elimination of VAT exemptions. Combined with the excise increases of 2010, this package should yield 1.2 percent of GDP annually.

20. We continue to implement our public finance reform program, which we view as an important prerequisite to ensuring transparency, discipline, efficiency and accountability in the public finance area. We progressed significantly in implementing our public finance reform action plan for 2009, which implies implementation of actions in a large number of areas, including developing liquidity management guidelines, preparing the functional and technical specification for the PFMS, developing a new format of the medium-term framework (Basic Data and Directions) document, improving the format of local budgets.

21. Public Finance Management Reform Action Plan 2010 has been approved and is under implementation. Consistently with the new budget code, by end-July 2010 we will seek cabinet approval of the medium-term expenditure framework (Basic Data and Directions) that includes expenditure ceilings applicable to the 2011 budget (structural benchmark under the program). Also, by end-September 2010 we intend to seek cabinet approval of guidelines for pilot Ministries to introduce a programmatic approach to budgeting, in line with the recommendations of the technical assistance from the IMF's Fiscal Affairs Department (structural benchmark).

22. In pursuance of our reforms aimed at fostering a culture of compliance and streamlining tax and customs administration procedures, we are in the process of discussing legal amendments to achieve cost-efficiency and effectiveness gains within the Revenue Service of the Ministry of Finance. These reforms would enhance the business-friendliness of tax and customs legislation with a view of further promoting private sector-led growth.

23. The monetary policy transmission channel has weakened due to the crises and remains inefficient under current excess liquidity conditions. To restore transmission channel NBG has made reforms to influence short-term interest more effectively. This includes restoring reserve requirements in local currency and activation of standing facilities.

24. In order to increase confidence in the NBG's refinancing loans and promote efficiency of the monetary policy, the NBG started to provide commercial banks with 
guaranteed access refinancing loans against collateral at the interest linked to the NBG's key policy rate. The collateral base for refinancing loans is extended by international bank guarantees and long term local currency loans. We expect the meet the end-June NDA target due to lower-than-expected demand for free reserves by banks. The NBG has begun to tighten monetary conditions by raising its refinancing rate from 5 to 6.25 percent on June 16 to maintain price stability as the economy recovers and to counter recent exchange rate pressures. We project end-period inflation to be 5 percent in 2010. This projection is, of course, sensitive to assumptions regarding the money multiplier and, in turn, commercial bank lending to the private sector, as well as international commodity price developments.

25. Intervention in the FX market in May and in June (to stabilize the market after an initial large depreciation) exceeded the room for intervention under the program, and the NIR target of end-June will likely be missed. Corrective measures have since been taken to reduce pressures, including faster exchange rate depreciation, monetary tightening and the fiscal measures described above. The commitment to a flexible exchange rate is unchanged and will be strengthened by reducing the frequency of auctions. The NBG does not target any exchange rate and will intervene only to smooth extreme volatility, to counter speculative pressures and if too fast and too large depreciation threatens financial stability. Should external developments be more favorable than currently projected, we stand ready to raise the NIR targets for future reviews, so as to strengthen our exit strategy from official balance of payments support.

26. Our current strategy is based on the expected recovery of private capital inflows, and FDI in particular, starting in the second half of 2010. To support that process we have corrected some of the administrative problems faced by investors at the beginning of the year. We consider that the successful completion of the elections also removes a major source of uncertainty for investors. At the same time, we are aware that the current strategy is subject to risks, and that a significant shortfall in the recovery of private capital inflows will require a substantial change in policies. In this regard, should we observe a failure of FDI to resume over the summer, we stand ready to further tighten monetary policy and to cut the budget deficit further, while allowing the exchange rate to adjust. In view of the adverse impact of significantly higher depreciation on the balance sheet of banks - through currency induced credit risk - we would rely to a large extent on monetary and fiscal policy tightening to preserve stability. In order to move swiftly and most efficiently on fiscal policy, we would implement budget cuts across the board at the level of ministries, thus allowing ministries to decide how best to implement the cuts within their budgets given their constraints and objectives.

27. Enhancing the competitiveness of the Georgian economy is, of course, of primary importance to us and is a key to reducing Georgia's external current account deficit to more sustainable levels over the medium term. Georgia's sound macroeconomic policies, its extremely favorable business environment and geographic advantages put it in a good 
position to benefit from a generalized recovery of FDI flows to emerging markets. We expect that additional structural reforms will be at the root of future competitiveness gains. These include further privatization and reductions of the state's role in the economy.

28. To bring our official statistics in line with international standards, we improved legal framework and reorganized the former State Department of Statistics (new title-Geostat). New Law on State Statistics strengthens the independence of the Geostat and ensures the sustainable production of official statistics. On May 17, 2010, we subscribed to the IMF's Special Data Dissemination Standard (SDDS), with a view to enhancing the availability of timely and comprehensive statistics provided to the public.

29. Based on our expectation of higher than projected budget support disbursements from IFIs in 2010, we will reduce the Government's use of IMF financing for the budget.

Accordingly, the purchase that would become available upon completion of the sixth review, would be the last purchase to be used for budget support in 2010 .

30. We have implemented the recommendations provided in the recent update of the Safeguards Assessment Report. In particular:

- The new Audit Committee Charter was elaborated based on the best international practice.

- The Audit Committee composition was modified and includes only non-executive members of the Board.

- The oversight function of the Audit Committee was strengthened.

- The assessment of internal audit activities for compliance with the International Standards for Professional Practice of Internal Auditing and The IIA's Code of Ethics was conducted.

- The Internal Audit Service reviewed operations of the newly implemented core banking system.

The internal audit of the new RTGS and reserve management systems will be carried out following their deployment.

\section{Program Monitoring}

31. We expect end-June performance criteria under the Stand-By Arrangement to be met, with the exception of the NIR target. Based on the corrective measures taken to reduce exchange rate pressures and to meet the program objectives of 2010, we request a waiver for non-observance of the end-June NIR performance criterion, and request the completion of the Sixth Review. We expect to meet the end-June performance criterion on the cash deficit of the general government but we are requesting a waiver of applicability since data to verify this PC will not become available before July 27. We will maintain our usual close policy dialogue with the Fund and are ready to take additional measures as appropriate to ensure that we meet program objectives. The Government will consult with the Fund on the 
adoption of these measures, and in advance of revisions to the policies contained in this letter, in accordance with the Fund's policies on such consultations.

32. We also request: (i) a modification of the end-September PCs on the fiscal deficit, NIR and NDA according to the attached Table 1, which also establishes end-December 2010 PCs; (ii) introduction of a PC on total government expenditure for end-September and EndDecember 2010; and (iii) rephasing of purchases by combining the seventh and the eighth reviews. The seventh and eighth reviews will be based on end-September 2010 performance criteria and are scheduled for completion by end-December 2010; and the ninth review will be based on end-December 2010 performance criteria and is scheduled for completion by end-March 2011. The revised Technical Memorandum of Understanding clarifies the measurement of the PC on government expenditure and of the indicative target on the contraction and guaranteeing of total government debt. Structural benchmarks under the program are described in Table 2.

33. We authorize the IMF to publish this Letter of Intent and its attachments as well as the accompanying staff report.

Sincerely yours,

/s/

Nika Gilauri

Prime Minister of Georgia
$/ \mathrm{s} /$

Kakha Baindurashvili

Minister of Finance

$/ \mathrm{s} /$

Giorgi Kadagidze

President of the National Bank of Georgia 
Table 1. Georgia: Quantitative Performance Criteria (PC) and Indicative Targets, 2010

\begin{tabular}{|c|c|c|c|c|c|c|c|c|c|}
\hline & \multicolumn{9}{|c|}{ Cumulative Change from End-December 2009} \\
\hline & \multicolumn{3}{|c|}{ Mar-10 } & \multicolumn{2}{|c|}{ Jun-10 } & \multicolumn{2}{|c|}{ Sep-10 } & \multicolumn{2}{|c|}{ Dec-10 } \\
\hline & PC & Adjusted PC & Actual & PC & Proj. & PC & Proposed PC & Ind. Target & Proposed PC \\
\hline & \multicolumn{9}{|c|}{ (In millions of lari) } \\
\hline Ceiling on cash deficit of the general government & 333 & & 96 & 737 & 467 & 1,089 & 867 & 1,393 & 1,241 \\
\hline \multirow[t]{2}{*}{ Ceiling on the general government expenditures } & $\ldots$ & & & $\ldots$ & $\ldots$ & $\ldots$ & 5,023 & $\ldots$ & 7,106 \\
\hline & \multicolumn{9}{|c|}{ (In millions of lari) } \\
\hline \multirow[t]{2}{*}{ Ceiling on net domestic assets (NDA) of the NBG 1/ } & 418 & 397 & 135 & 606 & 553 & 568 & 506 & 653 & 714 \\
\hline & \multicolumn{9}{|c|}{ (In millions of U.S. dollars) } \\
\hline Floor on net international reserves (NIR) of the NBG 1/ & 781 & 793 & 897 & 685 & 639 & 733 & 740 & 706 & 721 \\
\hline \multirow[t]{2}{*}{ Ceiling on accumulation of external arrears $2 /$} & 0 & & 0 & 0 & 0 & 0 & 0 & 0 & 0 \\
\hline & Ind. Target & & Actual & Ind. Target & Proj. & Ind. Target & Prop. Ind. Target & Ind. Target & $\begin{array}{c}\text { Prop. Ind. } \\
\text { Target }\end{array}$ \\
\hline $\begin{array}{l}\text { Ceiling on contracting or guaranteeing of new nonconcessional } \\
\text { external debt by the public sector } 3 /\end{array}$ & 850 & & 226 & 850 & $\begin{array}{l}\text { ns of } U \\
347\end{array}$ & 850 & $\ldots$ & 850 & .. \\
\hline $\begin{array}{l}\text { Ceiling on contracting or guaranteeing of new total external debt } \\
\text { by the public sector } 3 /\end{array}$ & $\ldots$ & & & $\ldots$ & $\ldots$ & $\ldots$ & 1,000 & $\ldots$ & 1,000 \\
\hline
\end{tabular}

Sources: Georgian authorities; and Fund staff estimates.

1/ Actual figures and quantitative targets are based on program exchange rates.

$2 /$ The continuous performance criterion for external arrears is defined in paragraph 17 of the TMU. 
Table 2. Georgia: Structural Benchmarks

Action

FSA and NBG to sign and implement a memorandum of understanding to strengthen cooperation.

NBG to introduce revised LOLR facility.

Submission to parliament of a state budget for 2009 with an overall fiscal deficit of no more than $33 / 4$ percent of projected GDP (subject to an adjustor of at most 3 percent of GDP as set out in the TMU).

NBG to develop and publish a liquidity management framework, including the introduction of a refinancing mechanism as the main instrument to provide liquidity.

The NBG, the FSA, and the government to develop a financial stability plan that will contain policy actions to respond to potential situations of stress by defining the roles and responsibilities of the different players-the NBG, the FSA and the government-under each circumstance.

Appointment of the remaining members to the FSA board.
Proposed

Time Frame

End-Oct-08

End-Dec-08

End-Dec-08

End-Jun-09

End-Jun-09

End-Jul-09

inclusive, enhance the budget execution monitoring process, and move to performance driven policy by improving strategic planning.

Status report on the implementation of the action plan described in the Financial Stability Plan, including bank-by-bank contingency measures based on stress test results and assurances of support from foreign shareholders.
Type of Conditionality

Status

Structural

Benchmark

Observed

Structural

Performance Criterion

Observed

Structural Benchmark

Observed

Not Structural observed. Benchmark Implemented with delay

Structural

Performance Criterion

Observed

Not observed, became

Structural redundant Benchmark with elimination of FSA board

Structural
Benchmark Observed


Submission to parliament of the Law on Internal Audit.

End-Sep-09 $\quad \begin{gathered}\text { Structural } \\ \text { Benchmark }\end{gathered}$

Submission to parliament of a state budget for 2010 that: (i) is consistent with the program targets and assumptions, and an overall deficit of no more than 7.3 percent of GDP (program definition); (ii) contains a medium-term fiscal framework consistent with the program's medium-term deficit reduction targets; and (iii) describes the medium-term policies underlying the fiscal deficit objectives through 2013.

Cabinet approval of a new medium-term expenditure framework (Basic Data and Directions) that includes expenditure ceilings applicable to the 2011 budget.

October 1, $2009 \quad \begin{gathered}\text { Structural } \\ \text { Benchmark }\end{gathered}$ Observed

Cabinet approval of guidelines for pilot Ministries to introduce a programmatic approach to budgeting in line with the recommendations of the IMF technical assistance report, "Georgia - Advancing Program Budgeting," by D. Radev, S. Flynn, L. Eyraud, and

Structural Benchmark 


\section{ATTACHMENT II. GeORgIA: REVISED TECHNICAL MEMORANDUM OF UNDERSTANDING}

June 24, 2010

1. This memorandum sets out the understandings between the Georgian authorities and the IMF staff regarding the definitions of quantitative performance criteria and indicative targets, as well as respective reporting requirements for the Stand-By Arrangement (SBA). It replaces the technical memorandum of understanding dated February 25, 2010.

2. These performance criteria and indicative targets are reported in Tables 1 attached to the Letter of Intent dated June 24, 2010. The exchange rate for the purposes of the program of the Georgian lari to the U.S. dollar is set at GEL $1.67=\$ 1$. The corresponding cross exchange rates are provided in Table 1.

\section{General Government and the Public Sector}

3. Definition: The general government is defined as the central government, local governments, and extra-budgetary funds. The public sector consists of the general government and the National Bank of Georgia (NBG).

4. Supporting material: The Treasury Department of the Ministry of Finance (MOF) will provide to the IMF detailed information on monthly revenues of the general government within two weeks of the end of each month, and monthly expenditures and arrears of the central government within four weeks of the end of each month. In addition, the Treasury will provide, on a daily basis, the cash balances in the accounts of the general government as of the previous business day.

\section{Quantitative Performance Criteria, Indicative Targets, and Continuous Performance Criteria: Definitions And Reporting Standards}

\section{A. Ceiling on the Cash Deficit of the General Government}

1. Definition: The cash deficit of the general government will be measured from the financing side at current exchange rates, and will be defined as equal to total financing. Total financing will be defined as the sum of (i) net domestic financing from banks and nonbanks, (ii) net external financing, and (iii) privatization receipts.

- Net domestic financing consists of bank and nonbank net financing to the general government which will be defined as follows:

(i) Net lending (borrowing net of repayments) provided by commercial banks to the general government plus the use of deposits held by the general government at commercial banks. Monitoring of net lending and government accounts will be based on the NBG's monetary survey and Treasury data. The change in cash balances of the 
local government at commercial banks for budget financing purposes will be monitored based on the "budget of territorial unit" account data provided by the Treasury Department. Any securities issued by the general government and purchased by commercial banks (for example, T-Bills) are also included in domestic financing.

(ii) Net lending (borrowing net of repayments) provided by the NBG to the general government plus the use of deposits of the general government held at the NBG. Monitoring of net lending and government accounts will be based on the Central Bank survey and Treasury data. The change in cash balances of the central government at the NBG for budget financing purposes will be monitored based on the "GEL TSA state budget" account data provided by the Treasury Department. Any securities issued by the general government and purchased by the NBG (for example, T-Bills) are also included in domestic financing.

(iii) Any securities issued by the general government and purchased by the nonbanks (for example, T-Bills or securitized claims on the government sold by the NBG) are also included in domestic financing.

- Net external financing is defined as the total of loans disbursed to the general government for budget support (including from the IMF), and project financing (capital expenditure and net lending), net change in external arrears, change in the accounts of the general government abroad, minus amortization. Amortization includes all external debt-related payments of principal by the general government.

- $\quad$ Privatization receipts consist of all transfers of monies received by the central and local governments in connection with the sale of central or local government assets. This includes receipts from the sale of shares, the sale of non-financial assets as well as leases and the sale of licenses with duration of 10 years and longer.

\section{Supporting material:}

- Data on domestic bank and nonbank financing will be provided to the IMF by the NBG and the Treasury Department of the MOF within four weeks after the end of the month.

- $\quad$ Data on external project financing as well as other external borrowing will be provided to the IMF monthly by the Debt Unit at the MOF (specifying projects by creditor) within two weeks of the end of each month.

- $\quad$ Data will be provided at the actual exchange rates. 
- Data on privatization receipts of the general government will be provided by the Treasury Department of the MOF to the IMF on a monthly basis within two weeks of the end of each month.

- Data on securitized debt sold by the NBG, including the securities that have been purchased by nonbanks, will be reported by the NBG on a monthly basis within two weeks of the end of each month.

\section{B. Ceiling on the General Government Expenditures}

3. Definition: The ceiling applies to total expenditures of the general government. Total expenditures include all current and capital spending as well as net lending: (i) current expenditures comprise compensation of employees, purchases of goods and services, subsidies, grants, social expenses, other expenses, other account payables and domestic and external interest payments; (ii) capital expenditures include projects financed by foreign loans and grants; (iii) net lending is defined as lending minus repayments to the general government. The Performance Criterion is monitored quarterly on a cumulative basis from the beginning of the year.

4. Supporting material: Data for monitoring expenditures will be derived from the accounts of the general government covered under the ceiling (based on state, local authority, and autonomous republics budgets). The ministry of finance is responsible for such reporting according to the above definition. Data on general government expenditures should be reported within four weeks after the end of the quarter.

\section{Floor on the Net International Reserves of the NBG}

5. Definition: For the program purposes, net international reserves (NIR) of the NBG in U.S. dollars are defined as foreign assets of the NBG minus the sum of foreign liabilities of the NBG. Foreign assets of the NBG include gold, gross foreign exchange reserves, Georgia's SDR holdings, and the reserve position in the IMF. Gross foreign exchange reserves of the NBG are defined as liquid, convertible currency claims of the NBG on nonresidents, including cash holdings of foreign exchange that are readily available. Pledged or otherwise encumbered assets, including (but not limited to) assets used as collateral (or guarantee for third party external liabilities) are excluded from foreign assets. Foreign liabilities of the NBG shall be defined as the sum of Georgia's outstanding liabilities to the IMF, Georgia's SDR allocation, and any other liabilities of the NBG, excluding the foreign exchange balances in the government's account with the NBG. Thus defined, the definition of NIR excludes foreign assets stemming from foreign currency deposits of financial institutions at the NBG and foreign assets arising from the currency swaps with financial institutions. For program monitoring purposes, the stock of foreign assets and foreign liabilities of the NBG shall be valued at program exchange rates as 
described in paragraph 2 above. The stock of NIR amounted to $\$ 879.8$ million as of December 31, 2008 (at the program exchange rate).

\section{Adjustors:}

The floor on the NIR of the NBG will be adjusted:

- (a) upward/downward by 50 percent for any excess/shortfall in the balance-ofpayments support loans and balance-of-payments support grants relative to the projected amounts presented in Table 2 .

- (b) upward/downward by 50 percent for any excess/shortfall in the disbursements of the project loans and project grants to the Treasury Single account at the NBG relative to the projected amounts presented in Table 2.

- (c) upward 100 percent for any shortfall in the amount of conversion for government imports relative to the projected amounts presented in Table 2.

7. Supporting material: Data on net international reserves (both at actual and program exchange rates); net foreign financing (balance of payments support loans, cash grants to the general government, amortization (excluding repayments to the IMF), interest payments on external debt by the MOF and the NBG); conversions for government imports and transfers of receipts from the Sovereign Wealth Funds will be provided to the IMF in a foreign exchange cash flow table (which include details of inflows, outflows, and net international reserves) on a weekly basis within three working days following the end of the week.

\section{Ceiling on Net Domestic Assets of the NBG}

8. Definition: Net domestic assets of the NBG are defined as the difference between reserve money and NIR as defined above in paragraph 9. Therefore, the ceiling on NDA is defined as projected reserve money (as defined in Table 3 ) minus the target NIR.

\section{Adjustors:}

The ceiling on the NDA of the NBG will be adjusted:

- (a) upward/downward by 50 percent for any shortfall/excess in the balance-ofpayments support loans and balance-of-payments support grants relative to the projected amounts presented in Table 2.

- (b) upward/downward by 50 percent for any shortfall/excess in the disbursements of the project loans and project grants to the Treasury Single account at the NBG relative to the projected amounts presented in Table 2.

- (c) downward 100 percent for any shortfall in the amount of conversion for government imports relative to the projected amounts presented in Table 2. 
10. Supporting material: The NBG will provide to the IMF its balance sheet, which includes data on reserve money and net domestic assets on a weekly basis within three working days following the end of the week. Data will be provided using both actual and program exchange rates.

\section{E. Ceiling on Contracting or Guaranteeing of New External Debt by the Public Sector}

11. Definition: External debt, based on the notion of the residency of the creditor, is defined as debt contracted by the public sector from lenders other than the IMF. This indicative target applies to debt as defined in point No. 9 of the IMF's Guidelines on Performance Criteria with Respect to External Debt as amended effective December 1, 2009 (Decision No. 12274-(00/85) August 24, 2000). ${ }^{1}$ Previously disbursed external debt that has been rescheduled will be excluded from the definition of "new debt" for the purposes of this performance criterion.

12. Supporting material: Details of all new contracted debt and government guarantees for external borrowing, with detailed explanations, will be provided by the MOF to the IMF on a quarterly basis within thirty days of the end of each quarter. Data will be provided using actual exchange rates.

\footnotetext{
${ }^{1}$ Point No. 9 of the IMF's guidelines reads as follows: "(a) For the purpose of this guideline, the term "debt" will be understood to mean a current, i.e., not contingent, liability, created under a contractual arrangement through the provision of value in the form of assets (including currency) or services, and which requires the obligor to make one or more payments in the form of assets (including currency) or services, at some future point(s) in time; these payments will discharge the principal and/or interest liabilities incurred under the contract. Debts can take a number of forms, the primary ones being as follows: (i) loans, i.e., advances of money to obligor by the lender made on the basis of an undertaking that the obligor will repay the funds in the future (including deposits, bonds, debentures, commercial loans and buyers' credits) and temporary exchanges of assets that are equivalent to fully collateralized loans under which the obligor is required to repay the funds, and usually pay interest, by repurchasing the collateral from the buyer in the future (such as repurchase agreements and official swap arrangements); (ii) suppliers' credits, i.e., contracts where the supplier permits the obligor to defer payments until sometime after the date on which the goods are delivered or services are provided; and (iii) leases, i.e., arrangements under which property is provided which the lessee has the right to use for one or more specified period(s) of time that are usually shorter than the total expected service life of the property, while the leaser retains the title to the property. For the purpose of the Guideline, the debt is the present value (at the inception of the lease) of all lease payments expected to be made during the period of the agreement excluding those payments that cover the operation, repair, or maintenance of the property. (b) Under the definition of debt set out in point 9(a) above, arrears, penalties, and judicially awarded damages arising from the failure to make payment under a contractual obligation that constitutes debt are debt. Failure to make payment on an obligation that is not considered debt under this definition (e.g., payment on delivery) will not give rise to debt."
} 


\section{F. Continuous Performance Criterion on Nonaccumulation of External Arrears}

13. Definition: During the period of the arrangement, the public sector will not accumulate any new external payment arrears on debt service obligations to official creditors. Official external payment arrears are defined as unpaid debt service by the consolidated government and the NBG beyond 30 days after the due date. The performance criterion on nonaccumulation of external debt is continuous.

14. Supporting material: Details of official arrears accumulated on interest and principal payments to creditors will be reported to the IMF within one week from the date of the missed payment. Data will be provided using actual exchange rates.

Table 1. Program Exchange Rates

\begin{tabular}{ccc}
\hline & Currency Name & Currency/US\$ \\
\hline SDR & Special Drawing Rights & 0.65 \\
GEL & Georgian lari & 1.67 \\
EUR & Euro & 0.72 \\
& & \\
\hline
\end{tabular}

\begin{tabular}{|l|c|c|c|}
\hline \multicolumn{3}{|c|}{ Table 2. Projected Balance-of-Payments Support Financing 1/ } \\
(In millions of U.S. dollars) \\
\hline & $\begin{array}{c}\text { Balance-of-payments support } \\
\text { loans and balance-of- } \\
\text { payments support grants }\end{array}$ & $\begin{array}{c}\text { Project loans and } \\
\text { project grants }\end{array}$ & $\begin{array}{c}\text { Conversion for } \\
\text { government } \\
\text { imports }\end{array}$ \\
\hline June 30, 2010 & 19.2 & 117.4 & 115.0 \\
\hline September 30, 2010 & 155.3 & 198.7 & 162.4 \\
\hline December 31, 2010 & 219.6 & 238.8 & 243.4 \\
\hline 1/ Cumulative from the beginning of the calendar year. & \\
\hline
\end{tabular}

\begin{tabular}{|l|c|}
\hline \multicolumn{2}{|c|}{$\begin{array}{c}\text { Table 3. Projected Reserve Money } \\
\text { (End-of-period stock in millions of lari) }\end{array}$} \\
\hline June 30, 2010 & $1,750.4$ \\
\hline September 30, 2010 & $1,741.4$ \\
\hline December 31, 2010 & $1,918.3$ \\
\hline
\end{tabular}




\section{INTERNATIONAL MONETARY FUND}

GEORGIA

Sixth Review Under the Stand-By Arrangement and Requests for Modification of Performance Criteria, Waiver of Nonobservance of Performance Criterion, Waiver of Applicability of Performance Criterion, and Rephasing of Purchase

\section{Informational Annex}

Prepared by the Middle East and Central Asia Department

June 24, 2010

Contents

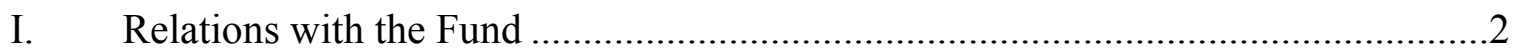

II. Relations with the World Bank ......................................................................

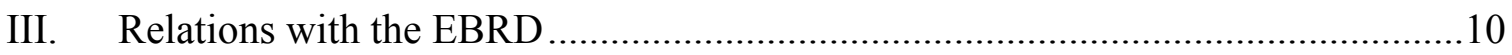




\section{ANNEX I. GEORGIA: RELATIONS WITH THE FUND}

(As of May 31, 2010)

I. Membership Status: Georgia joined the Fund on May 5, 1992.

II. General Resources Account:

Quota

Fund holdings of currency

Reserve position in Fund

III. SDR Department:

Holdings

IV. Outstanding Purchases and Loans:

Stand-by Arrangements

$\mathrm{ECF}^{1}$
SDR Million

150.30

677.40

0.01

SDR Million

144

SDR Million

527.10

114.15

Amount Approved $\underline{\text { (SDR million) }}$

747.10

98.00

108.00
Percent of Quota

100.00

450.70

0.01

Percent of Allocation

100.03

Percent of Quota

350.70

75.95

V. Latest Financial Arrangements:

Type

Approval

Stand-By

$\mathrm{ECF}^{1}$

$\mathrm{ECF}^{1}$
$9 / 15 / 08$

$6 / 4 / 04$

$1 / 12 / 01$
$6 / 14 / 11$

9/30/07

$1 / 11 / 04$
Amount Drawn

$\underline{\text { SDR Million) }}$

527.10

98.00

49.50

VI. Projected Payments to Fund (Expectation Basis):

(SDR million; based on existing use of resources and present holdings of SDRs):

\begin{tabular}{crrrrr} 
& \multicolumn{5}{c}{ Forthcoming } \\
\cline { 2 - 6 } & $\mathbf{2 0 1 0}$ & $\mathbf{2 0 1 1}$ & $\mathbf{2 0 1 2}$ & $\mathbf{2 0 1 3}$ & $\mathbf{2 0 1 4}$ \\
Principal & 9.15 & 38.11 & 159.90 & 244.86 & 141.75 \\
Charges/interest & 4.11 & 8.11 & 6.92 & 4.07 & 1.39 \\
Total & 13.26 & 46.23 & 166.82 & 248.93 & 143.14
\end{tabular}

\footnotetext{
${ }^{1}$ Following the Low Income Countries (LIC) reforms, effective January 7, 2010, the PRGF arrangements were renamed the Extended Credit Facility (ECF) Arrangements.
} 


\section{Safeguard Assessments:}

An update of the December 2008 safeguards assessment of the National Bank of Georgia (NBG) was completed on January 21, 2010 in conjunction with an augmentation of access under the SBA approved on August 6, 2009. The NBG continues to publish financial statements that comply with International Financial Reporting Standards, and are externally audited by an international firm in accordance with International Standards on Auditing. In response to the safeguards assessment, the authorities have agreed to a multi-year appointment of an external audit firm, beginning with financial year 2010.

\section{Implementation of Multilateral Debt Relief Initiative (MDRI):}

Not Applicable.

\section{Implementation of HIPC Initiative:}

Not Applicable.

\section{Exchange Arrangements:}

(a) Since April 29, 1993, the Tbilisi Interbank Currency Exchange (TICEX), established by the NBG and a group of commercial banks, has conducted periodic auctions to determine the exchange rate of the domestic currency vis-à-vis the U.S. dollar. These auctions are conducted daily. Foreign exchange bureaus are allowed to buy and sell foreign currency bank notes. A temporary de facto exchange rate peg to the U.S. dollar was introduced in the wake of the early August armed conflict. During the second week of November, the authorities allowed a 17 percent depreciation of the lari. In March 2009, the authorities introduced an auction-based system for the foreign exchange market. This mechanism is intended to allow more flexibility and give market forces a greater role in setting the price, with a view to reaching faster and smoother convergence toward equilibrium in the foreign exchange market and giving the market greater clarity about the authorities' policies. In end-May 2009, the authorities officially ended foreign exchange market interventions on TICEX.

(b) The government uses the official exchange rate for budget and tax accounting purposes as well as for all payments between the government and enterprises and other legal entities. The official rate may differ by more than two percent from freely determined market rates, which gives rise to a multiple currency practice.

\section{Article IV Consultation:}

The 2009 Article IV consultation was concluded on March 23, 2009. 


\section{FSAP Participation:}

Two FSAP missions visited Tbilisi during May 1-15, and July 24-August 7, 2001. An FSAP update mission visited Tbilisi during February 15-28, 2006.

\section{Technical Assistance:}

See Table 1 of this Annex.

\section{Resident Representative:}

The sixth resident representative, Mr. Edward Gardner, took up his post on March 15, 2009.

\section{National Bank of Georgia Resident Advisors:}

Ms. Vance, MAE peripatetic banking supervision advisor to the NBG, commenced a series of visits to Tbilisi in September 1997. Mr. Nielsen, an MAE advisor, provided technical assistance to the NBG in May 1998. Mr. Viksnins was an MAE peripatetic advisor to the NBG president starting in October 1999. Mr. Fish was resident advisor on banking supervision from August 10, 1999 to January 31, 2002. Mr. Bernard Thompson provided peripatetic technical assistance in accounting and internal audit in March and August 2000. Mr. Wellwood Mason provided technical assistance on payment system issues on a peripatetic basis in 2002 and 2003. Mr. Howard C. Edmonds served from September 2004 to October 2007 as a resident advisor on banking supervision issues.

\section{Ministry of Finance Resident Advisors:}

Mr. Sharma was an FAD resident advisor and assisted the authorities in the development of a Treasury beginning in May 1997. Mr. Sainsbury, an FAD advisor, assisted the ministry of finance from June 1998 to November 1999. Mr. Chaturvedi was FAD resident advisor in 2001 and 2002 to assist the authorities in continuing the development of the Treasury and the Treasury Single Account, in revising the legislative framework, expenditure control systems, and budgeting issues. Between 2001 and 2003, Mr. Welling was an FAD peripatetic advisor to assist the State Customs Department in preparing and introducing measures for the custom reform and modernization program. In March 2005, Mr. Zohrab started advising the authorities on treasury-related reforms, and his term ended in November 2006. 
Table 1. Georgia: Fund Technical Assistance Missions, 2004-09

\begin{tabular}{|c|c|c|c|}
\hline Subject & Type of Mission & Timing & Counterpart \\
\hline \multicolumn{4}{|c|}{ Fiscal Affairs Department (FAD) } \\
\hline Tax Policy & Review of tax policy & Jun. 8-21, 2004 & Ministry of Finance \\
\hline $\begin{array}{l}\text { Public Expenditure } \\
\text { Management }\end{array}$ & $\begin{array}{l}\text { Assessment of } \\
\text { Treasury system. }\end{array}$ & Nov. 8-23, 2004 & Ministry of Finance \\
\hline $\begin{array}{l}\text { Public Financial } \\
\text { Management }\end{array}$ & $\begin{array}{l}\text { Expert assistance } \\
\text { (Zohrab) }\end{array}$ & Mar. 2005-Nov. 2006 & Ministry of Finance \\
\hline Tax Administration & $\begin{array}{l}\text { Mission-review of } \\
\text { modernization } \\
\text { program }\end{array}$ & Nov. 8-21, 2005 & Ministry of Finance \\
\hline Tax Administration & Expert assistance & Jul. 9-14, 2006 & Ministry of Finance \\
\hline Tax Administration & $\begin{array}{l}\text { Expert assistance } \\
\text { (Bosch) }\end{array}$ & Oct. 9-25, 2006 & Ministry of Finance \\
\hline Tax Administration & Follow-up mission & Jan. 17-30, 2007 & Ministry of Finance \\
\hline Tax Administration & $\begin{array}{l}\text { Expert assistance } \\
\text { (Woodley) }\end{array}$ & $\begin{array}{l}\text { Jan/Feb and Apr/May } \\
2008\end{array}$ & Ministry of Finance \\
\hline $\begin{array}{l}\text { Budget Classification } \\
\text { and Accounting } \\
\text { Reforms }\end{array}$ & $\begin{array}{l}\text { Expert assistance } \\
\text { (Swarap) }\end{array}$ & Jun. 11-22, 2007 & Ministry of Finance \\
\hline $\begin{array}{l}\text { Budget Classification } \\
\text { and Accounting } \\
\text { Reforms }\end{array}$ & $\begin{array}{l}\text { Expert assistance } \\
\text { (Swarap) }\end{array}$ & Jun. 11-22, 2007 & Ministry of Finance \\
\hline $\begin{array}{l}\text { Public Financial } \\
\text { Management }\end{array}$ & Expert assistance & Apr. 6-19, 2010 & Ministry of Finance \\
\hline
\end{tabular}

Monetary and Capital Markets Department (MCM)

\begin{tabular}{|c|c|c|c|}
\hline $\begin{array}{l}\text { Government Securities } \\
\text { Market, Deposit } \\
\text { Insurance, Anti-Money } \\
\text { Laundering }\end{array}$ & Advisory & Apr. 20-30, 2004 & National Bank of Georgia \\
\hline $\begin{array}{l}\text { Monetary Operations, } \\
\text { Banking Sector } \\
\text { Competition }\end{array}$ & Advisory & $\begin{array}{l}\text { Oct. 24-Nov. 5, } \\
2004\end{array}$ & National Bank of Georgia \\
\hline $\begin{array}{l}\text { Liquidity Management; } \\
\text { Trends in Securities and } \\
\text { Insurance Sectors }\end{array}$ & Advisory & Apr. 18-29, 2005 & National Bank of Georgia \\
\hline FSAP Update & Assessment & Feb. 15-28, 2006 & National Bank of Georgia \\
\hline
\end{tabular}


Table 1. Georgia: Fund Technical Assistance Missions, 2004-09

\begin{tabular}{|c|c|c|c|}
\hline Subject & Type of Mission & Timing & Counterpart \\
\hline $\begin{array}{l}\text { Monetary } \\
\text { Operations/Monetary } \\
\text { Policy/Foreign } \\
\text { Exchange }\end{array}$ & Advisory & $\begin{array}{l}\text { Feb. 16-Mar. 2, } \\
2007\end{array}$ & National Bank of Georgia \\
\hline $\begin{array}{l}\text { Consolidated } \\
\text { Supervision }\end{array}$ & Advisory & Dec. 4-14, 2007 & National Bank of Georgia \\
\hline $\begin{array}{l}\text { Lender of Last Resort } \\
\text { Framework }\end{array}$ & Advisory & Dec. 11-17, 2008 & National Bank of Georgia \\
\hline $\begin{array}{l}\text { Stress Testing and } \\
\text { Foreign Exchange } \\
\text { Market }\end{array}$ & Advisory & Apr. 21-30, 2009 & $\begin{array}{l}\text { Financial Supervisory } \\
\text { Agency; National Bank of } \\
\text { Georgia }\end{array}$ \\
\hline $\begin{array}{l}\text { Macroeconomic } \\
\text { Modeling of Monetary } \\
\text { Policy Formulation }\end{array}$ & Advisory & June 2-10, 2010 & National Bank of Georgia \\
\hline \multicolumn{4}{|c|}{ Statistics Department (STA) } \\
\hline National Accounts & Follow-up assistance & Apr. 26-May 7, 2004 & $\begin{array}{l}\text { State Department of } \\
\text { Statistics }\end{array}$ \\
\hline National Accounts & Follow-up assistance & Apr. 18-29, 2005 & $\begin{array}{l}\text { State Department of } \\
\text { Statistics }\end{array}$ \\
\hline Price Statistics & Follow-up assistance & May 23-Jun. 3, 2005 & $\begin{array}{l}\text { State Department of } \\
\text { Statistics }\end{array}$ \\
\hline $\begin{array}{l}\text { Balance of Payments } \\
\text { Statistics/International } \\
\text { Investment Position }\end{array}$ & Follow-up assistance & Jun. 15-28, 2005 & $\begin{array}{l}\text { State Department of } \\
\text { Statistics and National } \\
\text { Bank of Georgia }\end{array}$ \\
\hline Price Statistics & Follow-up assistance & $\begin{array}{l}\text { Jun. 20-Jul. 13, } \\
2006\end{array}$ & $\begin{array}{l}\text { State Department of } \\
\text { Statistics }\end{array}$ \\
\hline Balance of Payments & Follow-up assistance & Sep. 6-19, 2006 & National Bank of Georgia \\
\hline $\begin{array}{l}\text { GDDS/Government } \\
\text { Finance Statistics }\end{array}$ & Follow-up assistance & Nov. 8-22, 2006 & $\begin{array}{l}\text { State Department of } \\
\text { Statistics, National Bank } \\
\text { of Georgia, Ministry of } \\
\text { Finance }\end{array}$ \\
\hline Balance of Payments & Follow-up assistance & Jul. 9-20, 2007 & National Bank of Georgia \\
\hline $\begin{array}{l}\text { Monetary and } \\
\text { Financial Statistics }\end{array}$ & Advisory & Mar. 18-31, 2009 & National Bank of Georgia \\
\hline \multicolumn{4}{|c|}{ Legal Department (LEG) } \\
\hline Tax Code & Follow-up assistance & $\begin{array}{l}\text { Jan. 28-Feb. 9, } \\
2001\end{array}$ & $\begin{array}{l}\text { Ministry of Finance, Tax } \\
\text { Inspectorate of Georgia }\end{array}$ \\
\hline Tax Code & Follow-up assistance & Jul. 13-24, 2001 & $\begin{array}{l}\text { Ministry of Finance, Tax } \\
\text { Inspectorate of Georgia }\end{array}$ \\
\hline
\end{tabular}




\section{ANNEX II. GEORGIA: RELATIONS WITH THE WORLD BANK}

(As of May 16, 2010)

1. Georgia has been a member of the World Bank since 1992 and the International Development Association (IDA) since 1993. Georgia has borrowed \$1,422 million for 60 operations (49 projects) to date. Up until FY09, all borrowings were IDA, but in FY09, Georgia became eligible for IBRD borrowings as well and is now a "blend" country. Georgia became a shareholder and member of the IFC in 1995.

2. A new CPS for Georgia for FY10-13 was endorsed by the World Bank Board in September 2009 against the backdrop of twin crises - the August conflict followed by the global economic downturn. As a result, the joint World Bank/IFC strategy focuses on key post-conflict and vulnerability issues in the near term, as well as strengthening the foundations for medium term competitiveness and growth. The CPS envisages Bank Group financing of about $\$ 740-900$ million over the four year period underpinned by a strong program of knowledge services. The financing envelope includes full use of the remaining IDA-15 envelope (projected at about \$130 million), indicative IBRD lending (of about \$266 million), disbursements under ongoing IDA/IBRD projects (of about \$135 million), and IFC investments and lending (of about \$210-360 million).

3. In response to the August conflict - and at the request of the Government - the World Bank in collaboration with other development partners prepared a Joint Needs Assessment (JNA) which was presented at the October 2008 Donors' Conference. The international community responded positively by pledging financial support totaling $\$ 4.5$ billion over the period of 2008-2011. This was meant to provide financial assistance to the country in the light of increased spending needs (on infrastructure, social sectors, banking, and general budgetary support), and a decline in capital inflows. To date, donors are on track to fulfilling their commitments.

4. The World Bank and the IMF have a strong track record of coordinated assistance to Georgia. The IMF has taken a lead on issues of macroeconomic policy, and the Bank and the Fund work together on financial sector strengthening. The World Bank has led the policy dialogue on social and structural policy issues, focusing on strengthening public expenditure policies and management, improving performance in the social sectors; poverty targeting and social assistance delivery, deepening and diversifying sources of growth; and rehabilitating infrastructure. The recent economic downturn has resulted in intensified collaboration on monitoring of macroeconomic developments and policy options.

5. Development policy lending has been a core element of Bank strategy and continues to be so in the current CPS. Following a successful past track record of reforms under the Poverty Reduction Support Operations, a programmatic series of three Development Policy 
Operations (DPOs) is underway under thecurrent CPS. The first DPO in amount of USD 85 million was approved on July 2, 2009. The DPO program aims at (i) mitigating the economic downturn; and (ii) facilitating recovery and preparing Georgia for post-crisis growth. It promotes policy and institutional reforms in (i) public finances, including improving efficiency and effectiveness of public expenditures, and public investment strengthening; (ii) social protection strengthening, including targeting and scaling up of the safety net system, pensions, and improving health coverage for the poor, and (iii) on specific measures to further improve the investment climate, including actions to modernize tax and customs administration. The second DPO in the amount of USD 50 million is expected to be presented to the Board in July 2010.

6. The Bank's program also includes strong support to strengthen connectivity, essential for Georgia's prospects as a transit corridor and spatially inclusive growth. This is primarily in the area of roads, through the Secondary and Local Roads Project and the East-West Highway Improvement Projects. In FY10, the Bank continued to support the East-West Highway program and as well as provide support on Kakheti regional roads. Donor coordination has been central to Bank strategy, especially in the context of the roads program where different donors are working together on a common platform of assistance.

7. Ongoing Bank-financed projects will continue to support progress in health, education, public sector, municipal development, and rural development. These include:

- In education, IDA credits are supporting education system realignment and strengthening which address a broad spectrum of educational reform issues, aimed at improving learning outcomes of primary and secondary students, and in rehabilitating schools.

- In health, an IDA-financed project is supporting the government in improving provision of primary health care services through the new Medical Insurance Program (MIP), providing training and equipment, and supporting the health information system as well as management capacity building.

- In rural development, IDA credits have supported development of private sector farming and agro-processing improvements, agricultural credit, irrigation and drainage, and agricultural research. IDA credits have also been supporting creation of local institutions, such as rural credit unions and water users associations.

- In public financial management, an IDA grant is pooled together with resources from other donors under the Public Sector Financial Management Reform Support Project to provide technical assistance and the necessary investments to support budget planning, execution, and overall management within the Ministry of Finance and line ministries. It also aims to strengthen public accountability and oversight of public financial management. 
- In municipal development, IDA is supporting the government to rehabilitate priority municipal infrastructure, support IDP housing, and strengthen intergovernmental fiscal arrangements.

8. Analytical and advisory activities across a broad spectrum of areas have been a central part of the Bank strategy. Significant pieces include the Progress Report on the JNA, a Poverty Assessment, an IFC Competitiveness study, a Country Procurement Assessment in 2007, and a joint WB-EU Public Expenditure and Financial Assessment in 2008. Ongoing work includes analysis of expenditure policy choices, work on regional trade integration, financial sector monitoring and risk assessment, programmatic poverty monitoring and analysis, a social sector review, continuing progress reports on the JNA, and energy sector work.

9. A key part of the Bank Group assistance has been to the private sector where IFC has financed projects in banking, infrastructure, oil and gas, and manufacturing sectors. IFC's committed portfolio in Georgia as of December 2009 stood at about $\$ 280$ million, of which $\$ 252$ million was disbursed. The portfolio includes 8 clients, primarily in the banking sector but also including several real sector clients. In the financial sector, IFC has focused on providing investment and technical assistance to three leading banks-TBC Bank, the Bank of Georgia, and Bank Republic. To reach small and medium enterprises, IFC helped to establish ProCredit Bank of Georgia, the country's first bank specializing in lending to micro and small enterprises. IFC has also provided a credit line to TBC Leasing to support the rapid growth of their portfolio of SME clients.

10. In the wake of the global financial crisis, IFC provided debt and equity investments to systemically important banks which enabled them to establish liquidity and capital cushions. In the real sector, IFC continues to look for opportunities to support the growth of the private sector, particularly in export-oriented companies or those that support overall private sector development. Sectors of interest include agrobusiness, infrastructure, manufacturing, and renewable energy. IFC has also provided technical assistance to strengthen its client banks and introduce new financial products (including leasing, housing finance and risk management products), and to improve the business climate and corporate governance practices. The World Bank-IFC Doing Business report has ranked Georgia as a top reformer in its Doing Business report, and the government is actively engaged with IFC to make further progress on reforms to improve the business environment.

Questions may be referred to Mr. A. Cholst (202-458-0324). 


\section{ANNEX III. GEORGIA: RELATIONS WITH THE EBRD}

(As of April 30, 2010)

1. As of April 30, 2010 the European Bank for Reconstruction and Development (EBRD) had signed 133 investments in Georgia with cumulative commitments totaling $\$ 1229.6$ million. ${ }^{1}$ Current Portfolio Stock equals to $\$ 898.5$ million. The EBRD’s first operation, a power rehabilitation project, was signed in December 1994. Since then, the pace and composition of portfolio growth has varied significantly from year to year.

2. During 2007 the Bank had signed 24 transactions in Georgia totaling \$282.4 million. ${ }^{2}$ The Bank provided a syndicated loan to ProCreditBank Georgia ( $\$ 8$ million); increased its equity participation in Republic Bank, introduced a medium size co-financing facility (MCFF) in Republic Bank and signed three sub operations under the facility in agriculture and health sectors (total $\$ 9.35$ million). MCFF has been successfully working for several years in one of the country's largest Bank TBC, with which the Bank signed six additional projects under the MCFF in agriculture ( $\$ 26$ million), general industry ( $\$ 1$ million) and natural resources ( $\$ 3$ million) sectors. The Bank financed mortgage lines to TBC Bank (\$12 million) and Republic Bank (\$4m) and provided loans to the Republic Bank (\$10 million), Cartu Bank (\$7 million) and Basis Bank (\$6 million) for on-lending purposes to medium, small and micro enterprises. The Bank introduced Energy Efficiency facility in two Georgian Banks: TBC Bank (\$10 million) and Cartu Bank (\$5 million).

3. The Bank's annual business volume in 2008 reached $\$ 302.6$ million through its 24 transactions. The project examples include: $\$ 7.5$ million loan to TBC Bank for development of SMEs, additional mortgage loan (\$20 million) to Republic Bank, the equity investment in Basis Bank, equity increase and subordinated debt (\$5 million) to Republic Bank. In 2008 the Bank signed three operations in municipal and environmental infrastructure development sector, namely: Batumi Public transport (\$3.5 million), Kobuleti (\$2.1 million) and Borjomi (\$2.1 million) water supply improvement projects. In October 2008 the Bank provided $\$ 14$ million loan to the company Geo Steel to finance construction and operation of $175 \mathrm{k}$ ton capacity steel mini-mill. In December 2008 the EBRD, along with the IFC provided Bank of Georgia, the largest bank in the country, with $\$ 200$ million loan facilities comprising of convertible subordinated, non-convertible subordinated and senior loans intended to support Bank of Georgia during a post-war period.

\footnotetext{
${ }^{1}$ EUR/USD rate at end of April 2010: 1.3258.

${ }^{2}$ EUR/USD rate at end of period: 1.4716 (Dec 2007); 1.4058 (Dec 2008); 1.4419 (Dec 2009).
} 
4. In 2009 the Bank has signed fifteen projects in private sector arriving at the business volume of $\$ 116.2$ million. As a response to the global financial crisis, EBRD, IFC and FMO joined forces to provide TBC Bank, with a funding package of $\$ 161$ million in loans and equity investment. The EBRD contribution is worth up to $\$ 70$ million and includes the acquisition of an equity stake for $\$ 36.8$ million and the provision of an $\$ 18.5$ million subordinated loan and a $\$ 14.7$ million senior loan. In addition, the EBRD provided $\$ 5$ million loan to Cartu bank for on lending to small and medium sized enterprises. The Bank signed six projects of $\$ 16.1$ million in agribusiness sector and four projects-in telecoms, general industry, property \& tourism and infrastructure sectors.

5. In 2010 the Bank signed the largest investments in Georgia - Tbilisi Railway By-Pass (\$132.6 million) and a sovereign guaranteed loan Black Sea Electricity Transmission Line (\$106.1 million); as well as Poti Port investment (\$10.6 million) and Adjara Solid Waste Landfill project (\$4 million).

6. The ratio of private sector projects in the portfolio now stands at 68.3 percent. The Bank plans to focus primarily on private sector financing, but may also consider selected public sector projects. The Bank will give preference to non-sovereign operations although, where sovereign guarantees are required, donor co-funding on a grant basis will be sought.

7. Georgia is part of the 'Early Transition Countries' (ETC) initiative. Launched in April 2004, the initiative aimed to increase investments in the Bank's seven poorest countries. The initiative builds on international efforts to address poverty in these countries. Through this initiative, the EBRD focuses its efforts on private sector business development and selected public sector interventions. It aims to stimulate market activity by using a streamlined approach to financing, focusing on smaller projects, mobilizing more investment, and encouraging ongoing economic reform. The Bank will accept higher risk in the projects it finances in the ETCs, while still respecting the principles of sound banking. Since launch of the ETC initiative, the Bank's annual business volume in Georgia has been increased five times. 
Table 1. Georgia: EBRD Portfolio for Georgia

(As of April 30, 2010)

\begin{tabular}{|c|c|c|c|}
\hline Project Name & $\begin{array}{c}\text { Date of } \\
\text { Agreement }\end{array}$ & $\begin{array}{c}\text { Outstanding } \\
\text { Amount } \\
\text { US Million } \\
\end{array}$ & $\begin{array}{c}\text { Outstanding } \\
\text { Amount } \\
\text { EUR Million }\end{array}$ \\
\hline Adjara Solid Waste Project & 18-Feb 2010 & 4.0 & 3.0 \\
\hline Bank Republic - MSE Loan & 27-Apr 2006 & 1.3 & 1.0 \\
\hline Bank Republic - MSME & 12-Dec 2008 & 10.0 & 7.5 \\
\hline Bank Republic Equity & 15-Sep 2006 & 7.8 & 5.9 \\
\hline Bank Republic Equity (Capital Increase) & 23-Apr 2008 & 1.3 & 1.0 \\
\hline Bank Republic Mortgage Loan & 20-Dec 2006 & 6.8 & 5.1 \\
\hline Bank Republic Mortgage Loan II & 22-Aug 2008 & 14.0 & 10.6 \\
\hline Bank Republic Subordinated Debt & 07-Jul 2008 & 5.0 & 3.8 \\
\hline Bank of Georgia - convertible subordinated debt & 30-Dec 2008 & 26.0 & 19.6 \\
\hline Bank of Georgia - subordinated debt & 30-Dec 2008 & 24.0 & 18.1 \\
\hline Bank of Georgia Senior Loan & 30-Dec 2008 & 50.0 & 37.7 \\
\hline $\begin{array}{l}\text { Basis Bank Equity Investment - Georgian Financial Sector } \\
\text { F/W }\end{array}$ & 19-May 2008 & 3.9 & 3.0 \\
\hline Batumi Public Transport Project & 02-Sep 2008 & 3.3 & 2.5 \\
\hline Black Sea Energy Transmission System & 17-Mar 2010 & 106.1 & 80.0 \\
\hline Borjomi Water Project & 01-Dec 2008 & 2.0 & 1.5 \\
\hline CEEP - Bank Republic & 19-Dec 2008 & 5.0 & 3.8 \\
\hline CEEP - Cartu Bank & 20-Dec 2007 & 4.9 & 3.7 \\
\hline CEEP - TBC & 30-Nov 2007 & 4.5 & 3.4 \\
\hline Cartu Bank SME Loan II & 30-Mar 2009 & 5.0 & 3.8 \\
\hline DIF - Alfapet & 08-Dec 2007 & 0.1 & 0.1 \\
\hline DIF - Delidor & 17-Jun 2005 & 1.4 & 1.1 \\
\hline DIF - Georgian Hazelnut Production Ltd. & 26-Oct 2006 & 0.8 & 0.6 \\
\hline DIF - Iberia Refreshments & 25-Sep 2003 & 3.2 & 2.4 \\
\hline DIF - Imedi L & 30-Dec 2006 & 1.5 & 1.2 \\
\hline DLF - BIH Castel & 23-Dec 2009 & 2.6 & 2.0 \\
\hline DLF - BTM TEKSTIL & 21-Dec 2006 & 1.5 & 1.2 \\
\hline DLF - Georgian Hazelnut Production Ltd & 26-Oct 2006 & 2.5 & 1.9 \\
\hline DLF - Populi & 31-Dec 2008 & 13.1 & 9.9 \\
\hline DLF - Margebeli & 23-Dec 2009 & 5.3 & 4.0 \\
\hline DLF - Marneuli & 24-Dec 2009 & 6.0 & 4.5 \\
\hline ETC Non-Bank Framework III - Crystal & 17-Dec 2009 & 0.5 & 0.4 \\
\hline ETC Non-Bank MFI Framework II - Constanta & 28-Nov 2006 & 1.6 & 1.2 \\
\hline ETC Non-Bank MFI Framework II - WV Credo & 07-Mar 2008 & 0.7 & 0.6 \\
\hline Enguri Hydro power Plant Rehabilitation project & 22-Dec 1998 & 20.6 & 15.6 \\
\hline Geo Steel & 20 -Oct 2008 & 14.0 & 10.6 \\
\hline Georgia: Tbilisi Railway Bypass Project & 17-Mar 2010 & 135.4 & 102.1 \\
\hline Georgia: Trans-Caucasian Rail Link Project & 22-Dec 1998 & 1.1 & 0.8 \\
\hline Georgian Financial Sector F/W - Basis Bank (MSME Loan) & 21-Sep 2007 & 5.1 & 3.9 \\
\hline Georgian Financial Sector F/W-Bank Republic MSE Loan & 21-Dec 2007 & 12.0 & 9.1 \\
\hline Georgian Financial Sector F/W-Cartu Bank SME Loan & 20-Dec 2007 & 6.0 & 4.5 \\
\hline Georgian Financial Sector FW-TBC Bank Mortgage Line II & 21-Dec 2007 & 11.3 & 8.5 \\
\hline Georgian Property & 29-Aug 2007 & 14.3 & 10.8 \\
\hline
\end{tabular}




\begin{tabular}{|c|c|c|c|}
\hline Georgian Property Debt FW - Tbilisi Central Train Station & 28-Dec 2007 & 8.5 & 6.4 \\
\hline Georgian Property Debt Facility - Green Building & 19-Mar 2007 & 2.3 & 1.7 \\
\hline Georgian Wines \& Spirits Ltd. & 10-Mar 2005 & 3.9 & 3.0 \\
\hline JSC Channel Energy Poti Port & 19-Mar 2002 & 1.4 & 1.1 \\
\hline Kobuleti Water & 27-Jun 2008 & 2.0 & 1.5 \\
\hline Kutaisi Water Project & 15-Sep 2006 & 3.9 & 2.9 \\
\hline MCFF Bank Republic - Rusmetali & 31-Dec 2009 & 2.5 & 1.9 \\
\hline MCFF - Bank Republic - Begi L/C and restructured loan & 10-Aug 2009 & 8.4 & 6.3 \\
\hline MCFF - Bank Republic - Full Recourse & 05-Dec 2008 & 10.0 & 7.5 \\
\hline MCFF - Bank of Georgia - GPC & 11-Feb 2010 & 2.4 & 1.8 \\
\hline MCFF - Bank of Georgia Full Recourse Portion & 14-Jun 2005 & 3.6 & 2.7 \\
\hline MCFF - BoG Iberia Refreshments Sub-Loan (NRP) & 27-Sep 2006 & 2.4 & 1.8 \\
\hline MCFF - TBC - Goodwill & 22-Dec 2009 & 0.8 & 0.6 \\
\hline MCFF - TBC - Tsiskvilkombinati II & 21-Nov 2007 & 1.5 & 1.1 \\
\hline MCFF - TBC Bank - Jaokeni Company JSC & 04-Aug 2008 & 2.2 & 1.7 \\
\hline MCFF - TBC Bank EMBAWOOD Sub Loan & 13-Jun 2007 & 0.6 & 0.4 \\
\hline MCFF - TBC Bank JSC Full Recourse Portion & 13-Jun 2005 & 1.4 & 1.1 \\
\hline MCFF - TBC Bank Tegeta Motors & 01-Apr 2008 & 6.2 & 4.7 \\
\hline MCFF - TBC Bank Wissol Sub-Loan (NRP) & 10 -Oct 2007 & 3.8 & 2.8 \\
\hline MCFF - TBC Coca Cola Ltd. sub-loan & 02-Feb 2007 & 3.4 & 2.6 \\
\hline MCFF - TBC Tsiskvilkombinati & 21-Nov 2007 & 1.5 & 1.2 \\
\hline $\begin{array}{l}\text { MCFF BOG - Renewable Energy Programme - Okami } \\
\text { SHPP NRP }\end{array}$ & 21-Dec 2006 & 0.1 & 0.1 \\
\hline $\begin{array}{l}\text { MCFF BOG - Renewable Energy Programme Lopota SHPP } \\
\text { NRP }\end{array}$ & 21-Dec 2006 & 0.1 & 0.1 \\
\hline MCFF Bank Republic - Mediclub Georgia & 30-Oct 2007 & 2.4 & 1.8 \\
\hline MCFF TBC Bank - Goodwill & 31-Oct 2007 & 6.3 & 4.8 \\
\hline MCFF-Bank Republic - BCFS & 18-May 2009 & 1.5 & 1.1 \\
\hline Poti Port: Phase 1 & 16-Mar 2010 & 10.6 & 8.0 \\
\hline Poti Water Supply Project & 15-Sep 2006 & 3.2 & 2.4 \\
\hline Regional TFP Basisbank (Guarantee \& Pre-export) & 06-Nov 2007 & 0.9 & 0.6 \\
\hline Regional TFP: Bank Republic (Guarantee \& Pre-export) & 15-Feb 2006 & 0.0 & 0.0 \\
\hline Regional TFP: Bank of Georgia (Guarantee \& Pre-export) & 29-Jul 1999 & 7.6 & 5.7 \\
\hline Regional TFP: Cartu Bank & 28-Apr 2006 & 1.7 & 1.3 \\
\hline Regional TFP: TBC Bank (guarantee \& pre-export) & 17-Aug 1999 & 9.7 & 7.3 \\
\hline Regional TFP: VTB Bank Georgia & 24-Dec 2000 & 0.0 & 0.0 \\
\hline Rustavi Solid Waste Management Project & 02-Jun 2009 & 2.1 & 1.6 \\
\hline TBC Bank & 07-Apr 2009 & 36.6 & 27.6 \\
\hline TBC Bank - Senior Loan & 14-Apr 2009 & 14.7 & 11.1 \\
\hline TBC Bank Mortgage Loan & 29-Jun 2006 & 8.3 & 6.3 \\
\hline TBC Bank SME Credit Line III & 26-Sep 2005 & 1.2 & 0.9 \\
\hline TBC Bank SME Line & 29-Jul 2008 & 7.5 & 5.7 \\
\hline TBC Bank: Subordinated Loan & 14-Apr 2009 & 18.5 & 14.0 \\
\hline TBC Leasing - Equity Investment & 01-Mar 2006 & 0.1 & 0.1 \\
\hline TBC Leasing, Senior Debt & 21-Dec 2005 & 0.9 & 0.6 \\
\hline Tbilisi International Airport & 17-May 2006 & 20.9 & 15.8 \\
\hline Tbilisi Public Transport Project & 29-Jul 2005 & 1.1 & 0.8 \\
\hline VTB Bank Georgia & $09-O c t 2006$ & 6.7 & 5.0 \\
\hline VTB Bank Georgia (debt, equity) & 20-Nov 1997 & 2.4 & 1.8 \\
\hline VTB Bank Georgia Capital Increase & 09-Oct 2006 & 2.3 & 1.8 \\
\hline Sub Total & & 785.9 & 592.7 \\
\hline
\end{tabular}




\begin{tabular}{|c|c|c|c|}
\hline Regional & & & \\
\hline Aureos Central Asia Fund LLC & 01-Dec 2008 & 0.8 & 0.6 \\
\hline $\mathrm{BIH}$ & 18-Dec 2006 & 0.0 & 0.0 \\
\hline BSR Europe Co-Investment Facility & 14-Aug 2006 & 9.3 & 7.0 \\
\hline Baku-Tbilisi-Ceyhan (BTC) Pipeline & 03-Feb 2004 & 45.1 & 34.0 \\
\hline Baring Vostok Private Equity Fund & 13-Dec 2000 & 0.0 & 0.0 \\
\hline Caucasus Online & 22-Dec 2008 & 30.6 & 23.1 \\
\hline Caucasus Online & 09-Oct 2009 & 0.9 & 0.7 \\
\hline Lukoil Overseas : South Caucasus Gas Pipeline & 28-Jul 2005 & 25.9 & 19.6 \\
\hline Regional Total & & 112.7 & 85.0 \\
\hline GEORGIA TOTAL & & 898.5 & 677.7 \\
\hline
\end{tabular}




\section{Statement by the IMF Staff Representative on Georgia \\ July 9, 2010}

1. The following information has become available since the issuance of the staff report. These developments do not change the thrust of the staff appraisal.

Georgia: Quantitative Performance Criteria (PC) and Indicative Target, June 2010

\begin{tabular}{|c|c|c|c|}
\hline & \multirow{2}{*}{\multicolumn{3}{|c|}{ Cumulative Change from End-December 2009}} \\
\hline & & & $\begin{array}{c}\text { Jun-10 } \\
\end{array}$ \\
\hline & \multicolumn{3}{|c|}{ Adjusted } \\
\hline & $\mathrm{PC}$ & $\mathrm{PC}$ & Actual \\
\hline & \multicolumn{3}{|c|}{ (In millions of lari) } \\
\hline \multirow[t]{2}{*}{ Ceiling on cash deficit of the general government $1 /$} & 737 & & $\ldots$ \\
\hline & \multicolumn{3}{|c|}{ (In millions of lari) } \\
\hline \multirow[t]{2}{*}{ Ceiling on net domestic assets (NDA) of the NBG 2/ } & 606 & 586 & 579 \\
\hline & \multicolumn{3}{|c|}{ (In millions of U.S. dollars) } \\
\hline Floor on net international reserves (NIR) of the NBG 2/ & 685 & 697 & 648 \\
\hline \multirow[t]{3}{*}{ Ceiling on accumulation of external arrears $3 /$} & 0 & & 0 \\
\hline & \multicolumn{3}{|c|}{ Ind. Target } \\
\hline & \multicolumn{3}{|c|}{ (In millions of U.S. dollars) } \\
\hline $\begin{array}{l}\text { Ceiling on contracting or guaranteeing of new nonconcessional } \\
\text { external debt by the public sector }\end{array}$ & 850 & & 347 \\
\hline \multicolumn{4}{|l|}{ Sources: Georgian authorities; and Fund staff estimates. } \\
\hline \multicolumn{4}{|c|}{$\begin{array}{l}\text { 1/ The cash deficit data will not be available until July } 27 \text {. } \\
2 / \text { Actual figures and quantitative targets are based on program exchange rates. } \\
3 / \text { The continuous performance criterion for external arrears is defined in paragraph } 17 \text { of the Technical }\end{array}$} \\
\hline
\end{tabular}

2. The end-June NDA PC was met while the NIR PC was missed as expected. The level of new nonconcessional external debt contracted or guaranteed by the public sector was well within the ceiling set as an indicative target.

3. Following the accelerated depreciation of early June, exchange rate pressures abated rapidly and the lari has since appreciated slightly due to a seasonal rebound of inflows. Cumulative depreciation vis-à-vis the dollar since the beginning of the year was 9 percent as of July 6. On June 24, the National Bank purchased \$10 million dollars in the foreign exchange market to slow the rate of appreciation and has not intervened since.

4. Real GDP growth was 4.5, year-on-year, in the first quarter of 2010, compared with staff projections of 3.2 percent. The program's annual real GDP growth projection of 4.5 percent for 2010 is therefore well within reach. CPI inflation dropped further to 3.7 percent in June, mainly on account of lower food prices.

5. Parliament has approved the supplementary budget for 2010 that is consistent with program targets. 
July 9,2010

\section{IMF Executive Board Completes Sixth Review Under the Stand-By Arrangement for Georgia}

The Executive Board of the International Monetary Fund (IMF) today completed the sixth review of Georgia's economic performance under a Stand-By Arrangement (SBA) for an amount equivalent to SDR 477.1 million (about US\$713.8 million) approved on September 15, 2008 (see Press Release No. 08/208). On August 6, 2009, the Executive Board approved an augmentation of access under the SBA to an amount equivalent to SDR 747.1 million (about US\$1,117.7 million) and an extension of the SBA until June 14, 2011 (see Press Release No. 09/277). The completion of the sixth review allows for the immediate purchase of an amount equivalent to SDR 50 million (about US\$74.8 million). The Executive Board granted a waiver for the nonobservance of the end-June 2010 performance criterion on the floor on the net international reserves of the National Bank of Georgia and a waiver of applicability of the end-June 2010 performance criterion on the ceiling on cash deficit of the consolidated government. The Executive Board also modified the performance criteria for end-September 2010.

After the Executive Board's discussion, Mr. Naoyuki Shinohara, Deputy Managing Director and Acting Chair, said:

"Georgia's economic recovery is gaining strength, backed by steady implementation of the program's economic policies. Nevertheless, risks remain, as evidenced by the recent weakness of FDI inflows and related exchange rate pressures. The authorities' prompt policy response, including faster depreciation and a tightening of monetary and fiscal policies, places Georgia in a good position to meet the objectives of the program, which are to secure macroeconomic stability and growth based on private sector financing and investment.

"The sizeable reduction in the fiscal deficit targeted for 2010-by nearly 3 percentage points of GDP-brings the objective of reaching a fiscally sound position within closer reach. The authorities' adoption of new revenue measures as well as their commitment to cap spending in 2010 is commendable. Moreover, the decision to postpone the implementation of a 
referendum requirement on tax increases will help maintain the necessary policy flexibility until the fiscal deficit has returned to more prudent levels. The authorities are encouraged to continue their efforts by targeting an ambitious reduction in the fiscal deficit in 2011.

"Bank lending shows signs of recovery, supported by a gradual lowering of lending interest rates over the past year. The tightening of monetary policy in June was warranted and the policy stance should continue to be adjusted promptly to changing market conditions.

"The banking sector's high levels of capital and provisioning continue to provide adequate buffers against adverse shocks, but continued close supervision of banks remains critical". 


\section{Statement by Age Bakker, Executive Director for Georgia And David Lezhava, Assistant to Executive Director \\ July 9, 2010}

My Georgian authorities would like to thank staff for the useful discussions held in Tbilisi last month and for an excellent report.

\section{Recent economic developments}

After the slowdown by 3.9 percent in 2009, the Georgian economy started to grow in 2010 . The annual growth rate in the first quarter was 4.5 percent and the same figure is expected to be achieved at the end of 2010. This is a significant increase in the forecast since the last review in March (2.0 percent).

Inflation remains well below the target rate (6 percent) and may stabilize at 5 percent throughout the rest of the year. The local currency has depreciated vis-à-vis the US dollar by 11.1 percent during the last 12 months; REER and NEER have depreciated by 7.0 and 6.5 percent respectively.

Acceleration in economic activity and increased competitiveness were reflected in the balance of payments figures. In the first quarter of 2010 the current account deficit decreased by 54.2 percent compared to the first quarter of 2009 as a result of increased exports and increased current transfers.

FDI in the first quarter of 2010 was US\$ 76 million, which is below expectations. This has been compensated by the implementation of official development assistance arrangements with multilateral and bilateral donors. The authorities believe that enhanced FDI inflows will come in the second half of 2010.

Against the backdrop of a general economic rebound observed in the first half of 2010 and as a result of revenue and expenditure-side measures, the budget deficit is expected to decline from a planned 7.4 percent of GDP to 6.3 percent of GDP (compared to 9.2 percent of GDP in 2009). The government aims at bringing the budget deficit below the 3 percent level by 2013.

The situation in the financial sector has steadily stabilized, while excess liquidity in the banking sector has gradually decreased. In 2010 credit started to grow. Local-currencydenominated loans increased by 25 percent in the first six months. The NPL ratio still remains relatively high at 17.6 percent according to the very conservative NBG definition (slightly less than 8 percent according to IMF definition). Other financial sector indicators remain satisfactory. The capital adequacy ratio is 17.6 percent as of the end of May. After a long sequence of losses, the banking sector has made gains for the last four months. 


\section{The program implementation}

Georgia has always observed program targets. All quantitative targets of March were met. We already know that end June quantitative targets were also met with the exception of the NIR target. Corrective measures have already been taken. The breach of the target can be explained because of special circumstances. The authorities observed excessive pressure on the FX market in April and May. The assumption was that this pressure was caused by expectations of political instability around upcoming local elections resulting in a slowdown of capital inflows. Furthermore, the National Bank's de-dollarization policy of providing local currency resources to commercial banks as a substitute for foreign borrowing could have, initially, created an excess supply of local currency on the FX market, although at a later stage demand for foreign currency would decrease. Hence, the National Bank believed in the temporary nature of the imbalance on the FX market and intervened heavily. Since the pressure persisted after the elections, the National Bank phased out intervention, in line with staff recommendations, and allowed a sharp depreciation. It also tightened monetary policy. The monetary policy rate was increased by 125 basis points. After exchange rate stabilization and the start of some appreciation the National Bank purchased back USD10 $\mathrm{mln}$ in the foreign exchange market

Some time is necessary to observe the full impact of the recent policy tightening, but, if economic developments so require it, additional tightening can be expected, accompanied by further purchases of foreign exchange. The National Bank is committed to allowing depreciation needed for adjustment; flexibility of the exchange rate in any case is consistent with the inflation-targeting strategy.

The Georgian authorities are thankful to IMF Board members for their comments and suggestions expressed during previous discussions. Many of past suggestions have been taken into account. My authorities have succeeded in mobilizing additional budget support and as agreed they have reduced correspondingly the amount of IMF financial support directed through the budget. Hence, they decided that, out of the SDR 150 million IMF financing considered under the program for the rest of 2010 only SDR 50 million will be channeled through the budget.

Concerns regarding the proposed referendum requirement for new taxes, expressed during previous Board discussions, were duly noted by my authorities, who have decided to postpone the implementation of this initiative until the budget deficit has been brought down to below 3 percent of GDP.

Georgia succeeded in conducting a peaceful and orderly election which reinforces political stability and provides the authorities with a strong mandate to continue the reforms.

The Ministry of Finance is implementing the Public Finance Management Reform Action Plan that includes approval of the medium-term expenditure framework and introduction of programmatic budgeting following the recommendations of technical assistance from the Fund's Fiscal Affairs Department. 
Both the monetary and fiscal authorities are taking steps for money market development. The National Bank increased the reserve requirements for commercial banks and took measures for absorbing access liquidity. This change is aimed at strengthening the effectiveness of monetary policy.

The monetary authorities are making successful efforts to reduce dollarization of the banking sector. They understand the complexity and time-demanding nature of the process. However, in the first few months of the process the share of local-currency loans in total loans has increased significantly from 23.2 to 26.4 percent (taking into account the revaluation effect due to the depreciation effect, the increase of local currency share is even stronger).

Among other achievements the following has to be noted:

- On May 17, 2010, Georgia subscribed to the IMF's Special Data Dissemination Standard (SDDS).

- Georgia has implemented the recommendations provided in the recent update of the Safeguards Assessment Report.

- In April 2010 S\&P's upgraded Georgia's sovereign rating.

We believe that program implementation has been successful and deserves Board approval of the review. Such decision will signal the international community's support to the economic policy of Georgia and may push the flow of dried-up private capital. 